\title{
An Effective Bifunctional Aldehyde Linchpin for Type II Anion Relay Chemistry (ARC): Development and Application to the Synthesis of a C16-C29 Fragment of Rhizopodin
}

Bruno Melillo, Ming Z. Chen, Roberto Forestieri and Amos B. Smith, III*

Department of Chemistry, Monell Chemical Senses Center and Laboratory for Research on the Structure of Matter, University of Pennsylvania, Philadelphia, PA 19104

*E-mail:smithab@sas.upenn.edu

\section{Supporting Information}

Experimental Procedures and Spectral Data 
General. All reactions were conducted in oven- or flame-dried glassware under a nitrogen atmosphere with anhydrous solvents, unless otherwise noted. Anhydrous diethyl ether, tetrahydrofuran and dichloromethane were obtained from a Pure Solve ${ }^{\mathrm{TM}}$ PS-400 solvent purification system. Hexamethylphosphoramide (HMPA) was purified prior to use by distillation over calcium hydride at 250 millitorr. $n$-Butyllithium was titrated using Love's method prior to use. ${ }^{1}$ All other commercially available reagents were used as received, unless otherwise noted. Reactions were monitored by thin-layer chromatography (TLC) with $250 \mu \mathrm{m}$ Silicycle pre-coated silica gel plates. Flash column chromatography was performed using ACS grade solvents and silica gel from Silicycle (P60, particle size 40-63 $\mu \mathrm{m}$ ). All compounds purified by chromatography were sufficiently pure for use in further experiments, unless otherwise indicated. Melting points were obtained on a Thomas-Hoover apparatus and are uncorrected. Infrared spectra were recorded on a Jasco Model FT/IR-480 Plus spectrometer. ${ }^{1} \mathrm{H}$ NMR and ${ }^{13} \mathrm{C}$ NMR spectra were recorded on a Bruker Avance III $500 \mathrm{MHz}$ spectrometer at 500 $\mathrm{MHz}$ and $125 \mathrm{MHz}$, respectively. ${ }^{1} \mathrm{H}$ NMR chemical shifts were reported relative to residual chloroform $(7.26 \mathrm{ppm})$ or benzene $(7.16 \mathrm{ppm})$ as follows: chemical shift $(\delta$, in ppm), splitting multiplicity ( $\mathrm{s}=$ singlet, $\mathrm{d}=$ doublet, $\mathrm{t}=$ triplet, $\mathrm{q}=$ =quartet, $\mathrm{qn}=$ quintet, $\mathrm{sxt}=$ sextet, $\mathrm{dd}=$ doublet of doublets, $\mathrm{ddd}=$ doublet of doublet of doublets, $\mathrm{dt}=$ doublet of triplets, $\mathrm{td}=$ =triplet of doublets, $\mathrm{m}=$ multiplet, br=broad, app=apparent), coupling constant $(\mathrm{Hz})$, and integration. ${ }^{13} \mathrm{C} \mathrm{NMR}$ chemical shifts were reported relative to chloroform $(77.16 \mathrm{ppm})$. High-resolution mass spectrometry (HRMS) was performed on either a Waters LC-TOF mass spectrometer (model LCT-XE Premier) or a Waters GCT Premier spectrometer at the University of Pennsylvania. Single crystal X-ray structures were determined on a Rigaku Mercury CCD or a Bruker APEXII CCD area detector employing graphite-monochromated Mo-Ka radiation $(1=0.71073 \AA)$ at a temperature of 143(1) K at the University of Pennsylvania. Supercritical fluid chromatography (SFC) analyses were performed with a JASCO system equipped with a Chiralpak AD-H column $(10 \mathrm{~mm} \times 250 \mathrm{~mm})$, a PU-280- $\mathrm{CO}_{2}$ plus $\mathrm{CO}_{2}$ Delivery System, a CO-2060 plus Intelligent Column Thermostat, an HC-2068-01 Heater Controller, a BP-2080 plus Automatic Back Pressure Regulator, an MD-2018 plus Photodiode Array Detector (200-648 nm), and PU-2080 plus Intelligent HPLC Pumps. 


\section{Synthesis of racemic aldehyde bifunctional linchpin 9:}

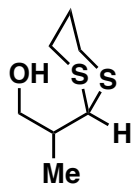

16

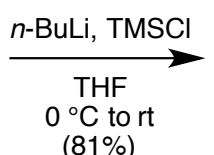

$(81 \%)$

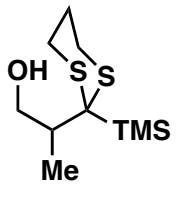

S-1

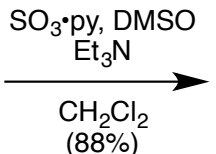

$(88 \%)$

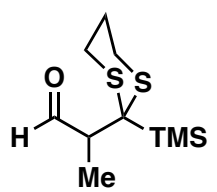

9

2-(1,3-dithian-2-yl)propan-1-ol (16) was prepared in 3 steps from 2-methyl-1,3-propanediol following literature procedures. ${ }^{2}$

2-(2-(trimethylsilyl)-1,3-dithian-2-yl)propan-1-ol (S-1). To a solution of 16 (2.0 g, $11.2 \mathrm{mmol})$ in THF $(22 \mathrm{~mL})$ at $0{ }^{\circ} \mathrm{C}$ was added dropwise $n$-BuLi (2.3 $\mathrm{M}$ in hexanes, $\left.10.7 \mathrm{~mL}, 24.7 \mathrm{mmol}\right)$. The resulting bright red solution was stirred for $30 \mathrm{~min}$, and then TMSCl $(3.13 \mathrm{~mL}, 2.68 \mathrm{~g}, 24.7$ mmol) was added slowly via a syringe. The reaction mixture was allowed to warm to $\mathrm{rt}$ as the red color faded rapidly to pale yellow. After stirring for an additional $1.5 \mathrm{~h}, 1.0 \mathrm{~N} \mathrm{HCl}(15 \mathrm{~mL})$ was added and the reaction was stirred for $30 \mathrm{~min}$. To work up, the reaction mixture was extracted with EtOAc $(3 \times 30 \mathrm{~mL})$. The combined organic extracts were washed with brine, dried over $\mathrm{Na}_{2} \mathrm{SO}_{4}$, filtered and concentrated in vacuo. The crude product was purified by flash column chromatography on silica gel (20\% EtOAc/hexanes) to afford TMS-dithiane alcohol S-1 $(2.28 \mathrm{~g}, 81 \%)$ as a white solid.

${ }^{1}$ H NMR $\left(500 \mathrm{MHz}, \mathrm{CDCl}_{3}\right) \delta 4.03(\mathrm{dd}, J=10.8,6.3 \mathrm{~Hz}, 1 \mathrm{H}), 3.62-3.55(\mathrm{~m}, 1 \mathrm{H}), 3.13(\mathrm{t}, J=$ $13.4 \mathrm{~Hz}, 1 \mathrm{H}), 2.98(\mathrm{t}, J=13.4 \mathrm{~Hz}, 1 \mathrm{H}), 2.91(\mathrm{~s}, 1 \mathrm{H}), 2.75(\mathrm{q}, J=6.7 \mathrm{~Hz}, 1 \mathrm{H}), 2.48-2.41(\mathrm{~m}, 2 \mathrm{H})$, 2.04 (app d, $J=13.4 \mathrm{~Hz}, 1 \mathrm{H}), 1.88(\operatorname{app} \mathrm{q}, J=12.9 \mathrm{~Hz}, 1 \mathrm{H}), 1.21$ (d, $J=7.5 \mathrm{~Hz}, 3 \mathrm{H}), 0.23$ (s, 9H);

${ }^{13}$ C NMR $\left(125 \mathrm{MHz}, \mathrm{CDCl}_{3}\right) \delta 67.4,42.6,42.1,24.5,24.2,23.8,17.1,-0.3$;

IR (thin film, NaCl) $v_{\max } 3304$ (br), 2949, 2904, 1456, 1420, 1247, 1021, 909, $843 \mathrm{~cm}^{-1}$; HRMS (EI, H) $m / z$ calc'd for $\mathrm{C}_{10} \mathrm{H}_{22} \mathrm{OS}_{2} \mathrm{Si}[\mathrm{M}+\mathrm{H}]^{+} 251.0960$, found 251.0963.

2-(2-(trimethylsilyl)-1,3-dithian-2-yl)propanal (9). To a solution of TMS-dithiane alcohol S-1 (4.86 g, $19.4 \mathrm{mmol})$ in $\mathrm{CH}_{2} \mathrm{Cl}_{2}(45 \mathrm{~mL})$ at $0{ }^{\circ} \mathrm{C}$ was added DMSO (7.72 mL, $8.49 \mathrm{~g}, 109 \mathrm{mmol}$ ) and $\mathrm{Et}_{3} \mathrm{~N}(14.3 \mathrm{~mL}, 10.4 \mathrm{~g}, 103 \mathrm{mmol})$. The resulting solution was cooled down to $0{ }^{\circ} \mathrm{C}$, and then $\mathrm{SO}_{3} \bullet$ py $(9.26 \mathrm{~g}, 58.2 \mathrm{mmol})$ was added portionwise. After stirring for $30 \mathrm{~min}$, the ice bath was removed, and the reaction was stirred for an additional $2.5 \mathrm{~h}$ at $\mathrm{rt}$. The reaction was then 
quenched with saturated aqueous $\mathrm{NH}_{4} \mathrm{Cl}$ solution $(70 \mathrm{~mL})$, and extracted with $\mathrm{CH}_{2} \mathrm{Cl}_{2}(3 \times 70$ $\mathrm{mL}$ ). The combined organic extracts were washed with brine, dried over $\mathrm{Na}_{2} \mathrm{SO}_{4}$, filtered and concentrated in vacuo. The crude product was purified by flash column chromatography on silica gel $(5 \rightarrow 10 \%$ EtOAc/hexanes) to afford aldehyde linchpin 9 (4.23 g, 88\%) as a pale yellow solid.

${ }^{1} \mathbf{H}$ NMR $\left(500 \mathrm{MHz}, \mathrm{CDCl}_{3}\right) \delta 9.88(\mathrm{~s}, 1 \mathrm{H}), 3.37-3.29(\mathrm{~m}, 1 \mathrm{H}), 2.96(\mathrm{t}, J=13.4 \mathrm{~Hz}, 2 \mathrm{H}), 2.44(\mathrm{t}$, $J=17.7 \mathrm{~Hz}, 2 \mathrm{H}), 2.07$ (app d, $J=14.4 \mathrm{~Hz}, 1 \mathrm{H}), 1.90($ app q, $J=13.0 \mathrm{~Hz}, 1 . \mathrm{H}), 1.28$ (d, $J=7.0$ $\mathrm{Hz}, 3 \mathrm{H}), 0.23$ (s, 9H);

${ }^{13}$ C NMR $\left(125 \mathrm{MHz}, \mathrm{CDCl}_{3}\right) \delta 202.7,52.1,38.9,24.4,23.6,23.1,14.2,-0.8$;

IR (thin film, NaCl) $v_{\max } 2952,2901,2831,2730,1714,1455,1423,1386,1250,1101 \mathrm{~cm}^{-1}$; HRMS (EI, H) $m / z$ calc'd for $\mathrm{C}_{10} \mathrm{H}_{20} \mathrm{OS}_{2} \mathrm{Si}[\mathrm{M}+\mathrm{H}]^{+} 249.0803$, found 249.0808.

\section{Tricomponent ARC fragment unions employing aldehyde bifunctional linchpin 9:}
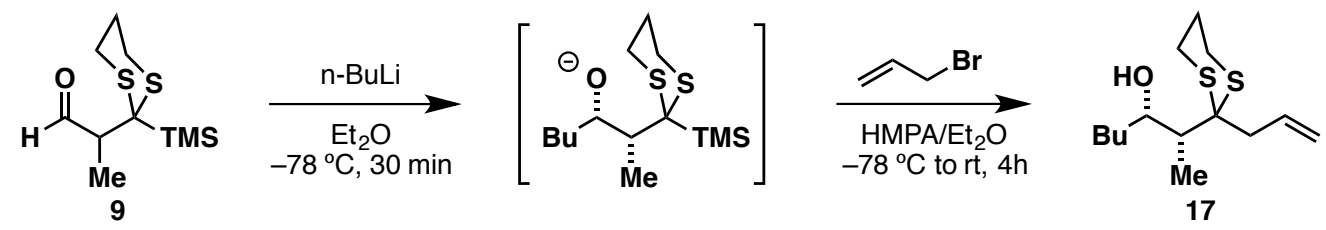

2-(2-allyl-1,3-dithian-2-yl)heptan-3-ol (17). To a solution of aldehyde linchpin 9 (124 mg, 0.5 $\mathrm{mmol})$ in $\mathrm{Et}_{2} \mathrm{O}(2.5 \mathrm{~mL})$ at $-78^{\circ} \mathrm{C}$ was added dropwise $n$-BuLi $(2.3 \mathrm{M}$ in hexanes, $261 \mu \mathrm{L}, 0.6$ mmol) via a syringe. After stirring for $30 \mathrm{~min}$, a solution of allyl bromide (173 $\mu \mathrm{L}, 242 \mathrm{mg}, 2.0$ $\mathrm{mmol})$ in $\mathrm{Et}_{2} \mathrm{O} / \mathrm{HMPA}(1.0 \mathrm{~mL}, 10 / 1, \mathrm{v} / \mathrm{v})$ was added dropwise. The resulting mixture was allowed to warm slowly to rt over $1 \mathrm{~h}$ and stirred for an additional $3 \mathrm{~h}$. The reaction was then quenched with $1.0 \mathrm{~N} \mathrm{HCl}(5 \mathrm{~mL})$ and stirred overnight. The reaction mixture was extracted with $\mathrm{Et}_{2} \mathrm{O}(3 \times 10 \mathrm{~mL})$. The combined organic extracts were washed with brine, dried over $\mathrm{MgSO}_{4}$, filtered and concentrated in vacuo. The crude product was purified by flash column chromatography on silica gel (5\% EtOAc/hexanes) to afford three-component adduct 17 (syn:anti $\geq 20: 1,102 \mathrm{mg}, 74 \%$ ) as a pale yellow oil. The syn to anti ratio was determined by ${ }^{1} \mathrm{H}$ NMR analysis of the crude reaction mixture, and the relative stereochemistry was assigned by analogy to adduct $\mathbf{2 1}$. 
${ }^{1}$ H NMR $\left(500 \mathrm{MHz}, \mathrm{CDCl}_{3}\right) \delta$ 5.93-5.83 (m, 1H), $5.14(\mathrm{~d}, J=24.5 \mathrm{~Hz}, 1 \mathrm{H}), 5.13(\mathrm{~s}, 1 \mathrm{H}), 4.41$ $(\operatorname{app~q}, J=4.0 \mathrm{~Hz}, 1 \mathrm{H}), 3.06(\mathrm{dd}, J=15.3,6.2 \mathrm{~Hz}, 1 \mathrm{H}), 3.01-2.91(\mathrm{~m}, 2 \mathrm{H}), 2.76(\mathrm{dd}, J=8.1$, $14.9,1 \mathrm{H}), 2.73-2.63(\mathrm{~m}, 2 \mathrm{H}), 2.31(\mathrm{~s}, 1 \mathrm{H}), 2.08-2.01(\mathrm{~m}, 1 \mathrm{H}), 1.91-1.84(\mathrm{~m}, 1 \mathrm{H}), 1.81(\mathrm{q}, J=6.4$ $\mathrm{Hz}, 1.56-1.48(\mathrm{~m}, 1 \mathrm{H}), 1.44-1.21(\mathrm{~m}, 5 \mathrm{H}), 1.06(\mathrm{~d}, J=7.1 \mathrm{~Hz}, 3 \mathrm{H}), 0.88(\mathrm{t}, J=6.9 \mathrm{~Hz}, 3 \mathrm{H})$; ${ }^{13}$ C NMR (125 MHz, $\left.\mathrm{CDCl}_{3}\right) \delta 134.1,118.0,70.9$, 57.7, 44.1, 41.0, 35.7, 28.7, 26.0, 26.0, 25.3, 22.8, 14.2, 7.0;

IR (thin film, NaCl) $v_{\max } 3466$ (br), 3074, 2931, 1713, 1637, 1455, 1379, 1271, 1080, $996 \mathrm{~cm}^{-1}$; HRMS (EI, H) $m / z$ calc'd for $\mathrm{C}_{14} \mathrm{H}_{26} \mathrm{OS}_{2} \mathrm{Na}[\mathrm{M}+\mathrm{Na}]^{+}$297.1317, found 297.1315.
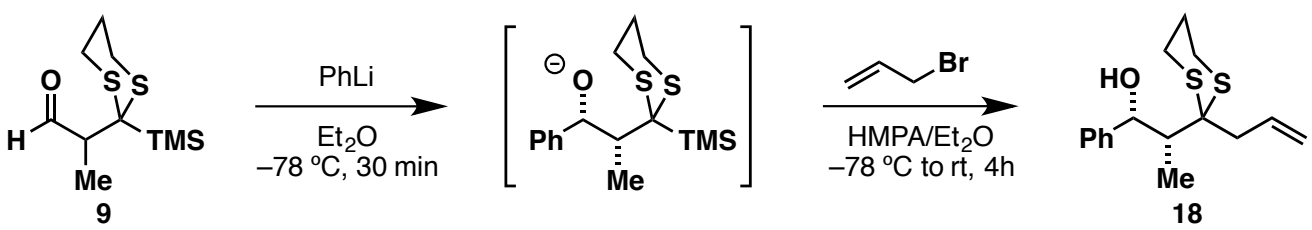

2-(2-allyl-1,3-dithian-2-yl)-1-phenylpropan-1-ol (18). To a solution of aldehyde linchpin 9 (124 mg, $0.5 \mathrm{mmol})$ in $\mathrm{Et}_{2} \mathrm{O}(2.5 \mathrm{~mL})$ at $-78^{\circ} \mathrm{C}$ was added dropwise $\mathrm{PhLi}(1.8 \mathrm{M}$ in dibutyl ether, $333 \mu \mathrm{L}, 0.6 \mathrm{mmol})$ via a syringe. After stirring for $30 \mathrm{~min}$, a solution of allyl bromide $(173 \mu \mathrm{L}$, $242 \mathrm{mg}, 2.0 \mathrm{mmol})$ in $\mathrm{Et}_{2} \mathrm{O} / \mathrm{HMPA}(1.0 \mathrm{~mL}, 10 / 1, \mathrm{v} / \mathrm{v})$ was added dropwise. The resulting mixture was allowed to warm slowly to $\mathrm{rt}$ over $1 \mathrm{~h}$ and stirred for an additional $3 \mathrm{~h}$. The reaction was then quenched with $1.0 \mathrm{~N} \mathrm{HCl}(5 \mathrm{~mL})$ and stirred overnight. The reaction mixture was extracted with $\mathrm{Et}_{2} \mathrm{O}(3 \times 10 \mathrm{~mL})$. The combined organic extracts were washed with brine, dried over $\mathrm{MgSO}_{4}$, filtered and concentrated in vacuo. The crude product was purified by flash column chromatography on silica gel (5\% EtOAc/hexanes) to afford three-component adduct 18 (syn:anti $\geq 20: 1,107 \mathrm{mg}, 73 \%$ ) as a white solid. The syn to anti ratio was determined by ${ }^{1} \mathrm{H}$ NMR analysis of the crude reaction mixture, and the relative stereochemistry was assigned by analogy to adduct $\mathbf{2 1}$.

${ }^{1}$ H NMR $\left(500 \mathrm{MHz}, \mathrm{CDCl}_{3}\right) \delta$ 7.39-7.30 (m, 4H), 7.25-7.20 (m, 1H), $5.97(\mathrm{td}, J=8.5,24.2 \mathrm{~Hz}$, 1H), $5.72(\mathrm{~s}, 1 \mathrm{H}), 5.18(\mathrm{~d}, J=24.2 \mathrm{~Hz}, 1 \mathrm{H}), 5.17(\mathrm{~s}, 1 \mathrm{H}), 3.15(\mathrm{dd}, J=15.3,6.2 \mathrm{~Hz}, 1 \mathrm{H}), 3.07-$ $2.95(\mathrm{~m}, 2 \mathrm{H}), 2.85-2.73(\mathrm{~m}, 4 \mathrm{H}), 2.13-2.05(\mathrm{~m}, 2 \mathrm{H}), 2.00-1.90(\mathrm{~m}, 1 \mathrm{H}), 0.98(\mathrm{~d}, J=7.1 \mathrm{~Hz}, 3 \mathrm{H})$;

${ }^{13}$ C NMR $\left(125 \mathrm{MHz}, \mathrm{CDCl}_{3}\right) \delta 144.3,134.0,128.1,126.7,125.7,118.2,72.3,57.5,47.4,41.2$, 26.1, 26.1, 25.2, 6.3;

IR (thin film, NaCl) $v_{\max } 3480$ (br.), 2904, 1635, 1558, 1540, 1418, 1065, 992, 915, $739 \mathrm{~cm}^{-1}$;

HRMS (EI, H) $m / z$ calc'd for $\mathrm{C}_{16} \mathrm{H}_{22} \mathrm{OS}_{2} \mathrm{Na}[\mathrm{M}+\mathrm{Na}]^{+} 317.1010$, found 317.1010. 

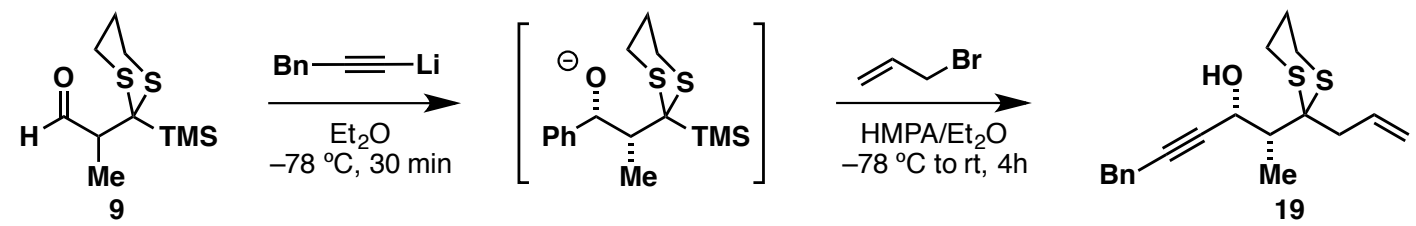

2-(2-allyl-1,3-dithian-2-yl)-6-phenylhex-4-yn-3-ol (19) Generation of nucleophile: to a precooled $\left(0{ }^{\circ} \mathrm{C}\right)$ solution of 3-phenyl-1-propyne $(0.093 \mathrm{~mL}, 0.750 \mathrm{mmol})$ in $\mathrm{Et}_{2} \mathrm{O}(2 \mathrm{~mL})$ was added n-butyllithium $(0.307 \mathrm{~mL}$ of a $2.4 \mathrm{M}$ solution in hexane, $0.750 \mathrm{mmol})$ dropwise. The cold bath was then removed and the reaction stirred at $\mathrm{rt}$ for $5 \mathrm{~min}$.

To a precooled $\left(-78^{\circ} \mathrm{C}\right)$ solution of $9(124 \mathrm{mg}, 0.5 \mathrm{mmol})$ in $\mathrm{Et}_{2} \mathrm{O}(2 \mathrm{~mL})$ was added the freshly prepared alkynyllithium dropwise via syringe (the original flask was rinsed with $\mathrm{Et}_{2} \mathrm{O}, 2 \times 0.25$ $\mathrm{mL})$. The mixture was stirred for $15 \mathrm{~min}$ at $-78{ }^{\circ} \mathrm{C}$, then a solution of allyl bromide $(173 \mu \mathrm{L}$, $2.00 \mathrm{mmol}) \mathrm{Et}_{2} \mathrm{O} / \mathrm{HMPA}(1.0 \mathrm{~mL}, 10 / 1, \mathrm{v} / \mathrm{v})$ was added dropwise. The resulting reaction mixture was stirred at $-78{ }^{\circ} \mathrm{C}$ for $1 \mathrm{~h}$, then the cold bath was removed and the reaction was stirred for an additional $1 \mathrm{~h}$. The reaction was then quenched with sat. aq. $\mathrm{NH}_{4} \mathrm{Cl}(1 \mathrm{~mL})$ and the biphasic mixture extracted with EtOAc $(3 \times 2 \mathrm{~mL})$. The organic extracts were combined, washed with brine, dried over $\mathrm{Na}_{2} \mathrm{SO}_{4}$, filtered and concentrated. The residue was taken up in THF (2 mL), 1 $N \mathrm{HCl}(2 \mathrm{~mL})$ was added, and the reaction was stirred at rt overnight. Sat. aq. $\mathrm{NaHCO}_{3}(2 \mathrm{~mL})$ was added, and the biphasic mixture extracted with EtOAc $(3 \times 2 \mathrm{~mL})$. The organic extracts were combined, washed with brine, dried over $\mathrm{Na}_{2} \mathrm{SO}_{4}$, filtered and concentrated. The residue was then purified by flash column chromatography (5\% EtOAc/hexanes) to furnish 19 as a clear colorless oil (110 mg, 66\%). The syn to anti ratio was determined by ${ }^{1} \mathrm{H}$ NMR analysis of the crude reaction mixture, and the relative stereochemistry was assigned by analogy to adduct 21.

${ }^{1}$ H NMR $\left(500 \mathrm{MHz}, \mathrm{CDCl}_{3}\right) \delta 7.38$ - $7.29(\mathrm{~m}, 4 \mathrm{H}), 7.27$ - 7.21 (m, $\left.1 \mathrm{H}\right), 5.95$ (dddd, J=6.2, 7.7, 10.4, 16.9 Hz, 1 H), 5.40 (dd, $J=1.8,3.8 \mathrm{~Hz}, 1 \mathrm{H}), 5.22-5.14$ (m, $2 \mathrm{H}), 3.66$ (d, J=1.8 Hz, 2 H), $3.11-3.00(\mathrm{~m}, 1 \mathrm{H}), 2.98-2.88(\mathrm{~m}, 2 \mathrm{H}), 2.85-2.76(\mathrm{~m}, 2 \mathrm{H}), 2.71(\mathrm{dd}, J=7.6,15.4 \mathrm{~Hz}, 1$ H), $2.57(\mathrm{~d}, J=4.2 \mathrm{~Hz}, 1 \mathrm{H}), 2.25(\mathrm{dq}, J=1.4,7.1 \mathrm{~Hz}, 1 \mathrm{H}), 2.02$ (qt, $J=3.4,10.3 \mathrm{~Hz}, 1 \mathrm{H})$, $1.98-1.88(\mathrm{~m}, 1 \mathrm{H}), 1.33(\mathrm{~d}, J=7.1 \mathrm{~Hz}, 3 \mathrm{H})$;

${ }^{13}$ C NMR $\left(125 \mathrm{MHz}, \mathrm{CDCl}_{3}\right) \delta 136.7,133.8,128.6,128.0,126.7,118.3,83.0,82.8,63.8,56.8$, 45.6, 41.0, 26.1, 26.1, 25.3, 25.0, 8.4;

IR (thin film, NaCl) $v_{\max } 3451$ (br), 2920, 1495, 1452, 1422, 1074, $988 \mathrm{~cm}^{-1}$;

HRMS (EI, H) $m / z$ calc'd for $\mathrm{C}_{19} \mathrm{H}_{24} \mathrm{OS}_{2} \mathrm{Na}[\mathrm{M}+\mathrm{Na}]^{+} 355.1166$, found 355.1170. 

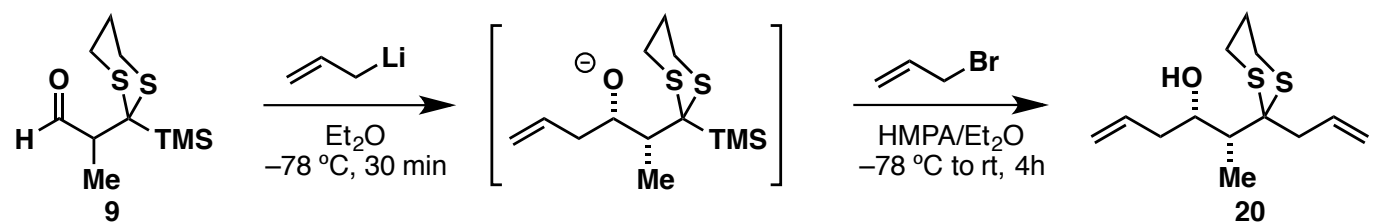

2-(2-allyl-1,3-dithian-2-yl)-1-phenylpropan-1-ol (20). Allyllithium was prepared freshly according to the literature procedure ${ }^{3}$ as follows: to a solution of allyltriphenylstannane (489 $\mathrm{mg}$, $1.25 \mathrm{mmol})$ in $\mathrm{Et}_{2} \mathrm{O}(3.0 \mathrm{~mL})$ at $\mathrm{rt}$ was added dropwise $\mathrm{PhLi}(1.30 \mathrm{M}$ in dibutyl ether, $577 \mu \mathrm{L}$, $0.75 \mathrm{mmol}$ ). The resulting mixture was stirred for $30 \mathrm{~min}$ as white solids precipitated out. The supernatant (containing allyllithium) was then cannulated dropwise to a solution of aldehyde linchpin $9(124 \mathrm{mg}, 0.5 \mathrm{mmol})$ in $\mathrm{Et}_{2} \mathrm{O}(2.0 \mathrm{~mL})$ at $-78{ }^{\circ} \mathrm{C}$. After stirring for $30 \mathrm{~min}$, a solution of allyl bromide $(173 \mu \mathrm{L}, 242 \mathrm{mg}, 2.0 \mathrm{mmol})$ in $\mathrm{Et}_{2} \mathrm{O} / \mathrm{HMPA}(1.0 \mathrm{~mL}, 10 / 1, \mathrm{v} / \mathrm{v})$ was added dropwise. The resulting mixture was allowed to warm slowly to $\mathrm{rt}$ over $1 \mathrm{~h}$ and stirred for an additional $3 \mathrm{~h}$. The reaction was then quenched with $1.0 \mathrm{~N} \mathrm{HCl}(5 \mathrm{~mL})$ and stirred overnight. The reaction mixture was extracted with $\mathrm{Et}_{2} \mathrm{O}(3 \times 10 \mathrm{~mL})$. The combined organic extracts were washed with brine, dried over $\mathrm{MgSO}_{4}$, filtered and concentrated in vacuo. The crude product was purified by flash column chromatography on silica gel (5\% EtOAc/hexanes) to afford threecomponent adduct $\mathbf{2 0}$ (syn:anti $\geq 20: 1,75 \mathrm{mg}, 58 \%$ ) as a pale yellow oil. The syn to anti ratio was determined by ${ }^{1} \mathrm{H}$ NMR analysis of the crude reaction mixture, and the relative stereochemistry was assigned by analogy to adduct 21 .

${ }^{1}$ H NMR (500 MHz, $\left.\mathrm{CDCl}_{3}\right) \delta 6.05$ - 5.75 (m, $\left.2 \mathrm{H}\right), 5.27$ - $4.97(\mathrm{~m}, 4 \mathrm{H}), 4.53(\mathrm{t}, J=6.7 \mathrm{~Hz}, 1$ H), $3.05(\mathrm{tdd}, J=1.3,6.3,15.3 \mathrm{~Hz}, 1 \mathrm{H}), 3.01$ - 2.91 (m, $2 \mathrm{H}), 2.82$ - 2.67 (m, $3 \mathrm{H}), 2.40$ - 2.29 (m, $2 \mathrm{H}), 2.19$ - 2.01 (m, $2 \mathrm{H}), 1.98$ - 1.83 (m, $2 \mathrm{H}), 1.11(\mathrm{~d}, J=6.9 \mathrm{~Hz}, 3 \mathrm{H})$;

${ }^{13}$ C NMR $\left(125 \mathrm{MHz}, \mathrm{CDCl}_{3}\right) \delta 135.5,134.1,118.0,117.4,70.2,57.7,43.3,41.0,40.7,26.1$, 26.0, 25.3, 7.1;

IR (thin film, $\mathrm{NaCl}$ ) $v_{\max } 3466$ (br), 2906, 1637, 1423, 1273, 915, $703 \mathrm{~cm}^{-1}$;

HRMS (EI, H) $m / z$ calc'd for $\mathrm{C}_{13} \mathrm{H}_{21} \mathrm{OS}_{2}[\mathrm{M}-\mathrm{H}]^{+} 257.1034$, found 257.1052. 

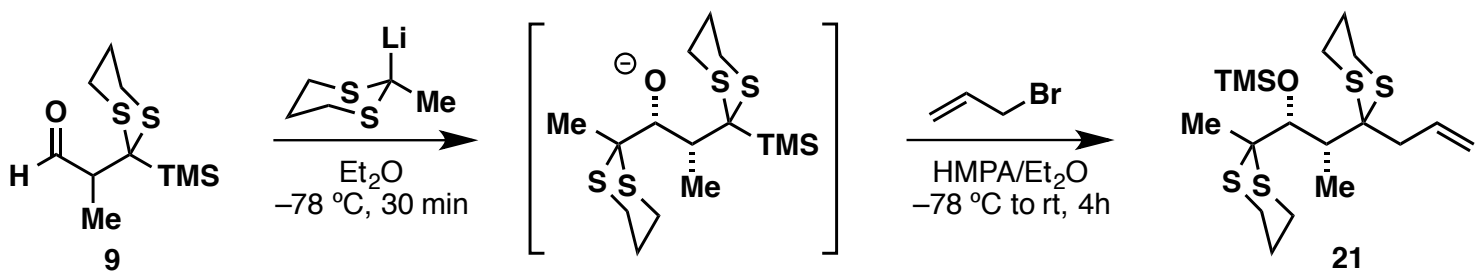

(2-(2-allyl-1,3-dithian-2-yl)-1-(2-methyl-1,3-dithian-2-yl)propoxy)tri-methylsilane (21). To a solution of aldehyde linchpin $9(124 \mathrm{mg}, 0.5 \mathrm{mmol})$ in $\mathrm{Et}_{2} \mathrm{O}(2.5 \mathrm{~mL})$ at $-78{ }^{\circ} \mathrm{C}$ was added dropwise lithiated 2-methyl-1,3-dithiane, generated in situ via deprotonation of 2-methyl-1,3dithiane $(90 \mu \mathrm{L}, 101 \mathrm{mg}, 0.75 \mathrm{mmol})$ with $n$-BuLi (2.08 $\mathrm{M}$ in hexanes, $360 \mu \mathrm{L}, 0.75 \mathrm{mmol})$. After stirring for $30 \mathrm{~min}$, a solution of allylbromide $(173 \mu \mathrm{L}, 242 \mathrm{mg}, 2.0 \mathrm{mmol})$ in $\mathrm{Et}_{2} \mathrm{O} / \mathrm{HMPA}$ $(1.0 \mathrm{~mL}, 10 / 1, \mathrm{v} / \mathrm{v})$ was added dropwise. The resulting mixture was allowed to warm slowly to $\mathrm{rt}$ over $1 \mathrm{~h}$ and stirred for an additional $3 \mathrm{~h}$. The reaction was then quenched with saturated aqueous $\mathrm{NH}_{4} \mathrm{Cl}$ solution $(5 \mathrm{~mL})$. The reaction mixture was extracted with $\mathrm{Et}_{2} \mathrm{O}(3 \times 10 \mathrm{~mL})$. The combined organic extracts were washed with brine, dried over $\mathrm{MgSO}_{4}$, filtered and concentrated in vacuo. The crude product was purified by flash column chromatography on silica gel (2\% EtOAc/hexanes) to afford three-component adduct 21 (syn:anti $\geq 20: 1,110 \mathrm{mg}$, $52 \%)$ as a white crystalline solid. mp $98-101^{\circ} \mathrm{C}$.

${ }^{1}$ H NMR $\left(500\right.$ MHz, $\left.\mathrm{CDCl}_{3}\right) \delta$ 6.07-5.97 (m, 1H), $5.16(\mathrm{~s}, 1 \mathrm{H}), 5.13(\mathrm{~s}, 1 \mathrm{H}), 4.67(\mathrm{~s}, 1 \mathrm{H}), 3.24-$ $3.16(\mathrm{~m}, 1 \mathrm{H}), 3.05-2.98(\mathrm{~m}, 1 \mathrm{H}), 2.92-2.84(\mathrm{~m}, 2 \mathrm{H}), 2.84-2.55(\mathrm{~m}, 7 \mathrm{H}), 2.04-1.85(\mathrm{~m}, 4 \mathrm{H}), 1.49$ (s, 3H), $1.05(\mathrm{~d}, J=6.9 \mathrm{~Hz}, 3 \mathrm{H}), 0.24(\mathrm{~s}, 9 \mathrm{H})$;

${ }^{13}$ C NMR $\left(125 \mathrm{MHz}, \mathrm{CDCl}_{3}\right) \delta 134.1,117.6,74.7$, 58.9, 55.6, 40.3, 40.1, 27.2, 27.0, 26.2, 26.0, 25.0, 24.7, 23.8, 11.0, 1.9;

IR (thin film, $\mathrm{NaCl}$ ) $v_{\max } 2900,1421,1244,1106,909,838 \mathrm{~cm}^{-1}$;

HRMS (EI, H) $m / z$ calc'd for $\mathrm{C}_{18} \mathrm{H}_{35} \mathrm{OS}_{4} \mathrm{Si}[\mathrm{M}+\mathrm{H}]^{+} 423.1335$, found 423.1338 .
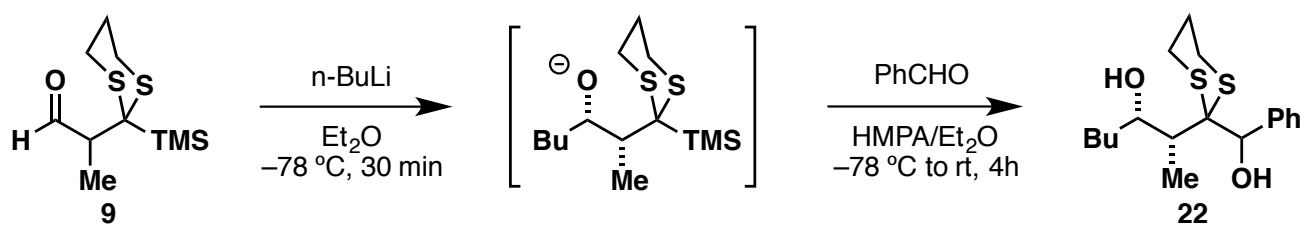

2-(2-(hydroxy(phenyl)methyl)-1,3-dithian-2-yl)heptan-3-ol (22) To a solution of aldehyde linchpin 9 (124 mg, $0.5 \mathrm{mmol})$ in $\mathrm{Et}_{2} \mathrm{O}(2.5 \mathrm{~mL})$ at $-78{ }^{\circ} \mathrm{C}$ was added dropwise $n$-BuLi $(2.08 \mathrm{M}$ in hexanes, $288 \mu \mathrm{L}, 0.6 \mathrm{mmol})$ via a syringe. After stirring for $30 \mathrm{~min}$, a solution of $(R)-1,2-$ 
epoxybutane ( $87 \mu \mathrm{L}, 72 \mathrm{mg}, 1.0 \mathrm{mmol})$ in $\mathrm{Et}_{2} \mathrm{O} / \mathrm{HMPA}(1.0 \mathrm{~mL}, 10 / 1, \mathrm{v} / \mathrm{v})$ was added dropwise. The resulting mixture was allowed to warm slowly to rt over $1 \mathrm{~h}$ and stirred for an additional $3 \mathrm{~h}$. The reaction was then quenched with sat. aq. $\mathrm{NH}_{4} \mathrm{Cl}(1 \mathrm{~mL})$ and the biphasic mixture extracted with EtOAc $(3 \times 2 \mathrm{~mL})$. The organic extracts were combined, washed with brine, dried over $\mathrm{Na}_{2} \mathrm{SO}_{4}$, filtered and concentrated. The residue was taken up in THF $(2 \mathrm{~mL}), 1 N \mathrm{HCl}(2 \mathrm{~mL})$ was added, and the reaction was stirred at rt overnight. Sat. aq. $\mathrm{NaHCO}_{3}(2 \mathrm{~mL})$ was added, and the biphasic mixture extracted with EtOAc $(3 \times 2 \mathrm{~mL})$. The organic extracts were combined, washed with brine, dried over $\mathrm{Na}_{2} \mathrm{SO}_{4}$, filtered and concentrated. The residue was then purified by flash column chromatography $\left(10 \% \rightarrow 15 \% \mathrm{Et}_{2} \mathrm{O} /\right.$ hexanes $)$ to furnish 22 as a clear colorless oil (syn:anti $\geq 20: 1,122 \mathrm{mg}, 72 \%$ ). The diastereomeric ratio and the syn to anti ratio were determined by ${ }^{1} \mathrm{H}$ NMR analysis of the crude reaction mixture, and the relative stereochemistry was assigned by analogy to adduct $\mathbf{2 1}$. The spectral data were obtained for the mixture of two sets of diastereomers.

${ }^{1} \mathbf{H}$ NMR $\left(500 \mathrm{MHz}, \mathrm{CDCl}_{3}\right) \delta 7.54-7.71(\mathrm{~m}, 4 \mathrm{H}), 7.22-7.40(\mathrm{~m}, 6 \mathrm{H}), 5.09(\mathrm{~s}, 1 \mathrm{H}), 4.94(\mathrm{~s}$, 1H), 4.51 (dd, $J=4.4,7.5 \mathrm{~Hz}, 1 \mathrm{H}), 4.46$ (dd, $J=5.3,7.4 \mathrm{~Hz}, 1 \mathrm{H}$ ), 3.62 (br. s, 4H), 2.37 - 2.67 (m, 7H), 2.19 - $2.34(\mathrm{~m}, 2 \mathrm{H}), 2.00-2.11(\mathrm{~m}, 1 \mathrm{H}), 1.94$ (q, $J=6.9 \mathrm{~Hz}, 1 \mathrm{H}), 1.80-1.90(\mathrm{~m}, 1 \mathrm{H}), 1.64$ - $1.79(\mathrm{~m}, 3 \mathrm{H}), 1.53-1.63(\mathrm{~m}, 1 \mathrm{H}), 1.45-1.53(\mathrm{~m}, 1 \mathrm{H}), 1.20-1.44(\mathrm{~m}, 10 \mathrm{H}), 1.17$ (d, $J=4.6$ $\mathrm{Hz}, 3 \mathrm{H}), 1.16(\mathrm{~d}, J=4.8 \mathrm{~Hz}, 3 \mathrm{H}), 0.91(\mathrm{t}, J=6.9 \mathrm{~Hz}, 3 \mathrm{H}), 0.87(\mathrm{t}, J=7.1 \mathrm{~Hz}, 3 \mathrm{H})$;

${ }^{13}$ C NMR (125 MHz, $\left.\mathrm{CDCl}_{3}\right) \delta 140.0,139.4,129.3,129.0,128.6,128.1,128.0,127.7,127.3$, $76.3,75.1,71.3,70.4,63.2,61.8,45.8,44.6,36.2,35.5,28.9,28.6,27.1,26.1,25.9,25.7,24.2$, $24.2,22.9,14.2,8.8,8.1$;

IR (thin film, NaCl) $v_{\max } 3366$ (br.), 3030, 2930, 1716, 1493, 1454, 1379, 1275, 1044, $910 \mathrm{~cm}^{-1}$; HRMS (EI, H) $m / z$ calc'd for $\mathrm{C}_{18} \mathrm{H}_{28} \mathrm{O}_{2} \mathrm{~S}_{2} \mathrm{Na}[\mathrm{M}+\mathrm{Na}]^{+} 363.1428$, found 363.1418 .
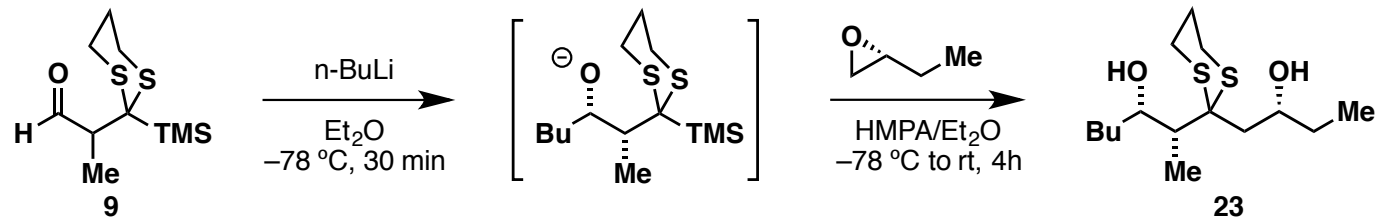

2-(2-((R)-2-hydroxybutyl)-1,3-dithian-2-yl)heptan-3-ol (23). To a solution of aldehyde linchpin 9 (124 mg, $0.5 \mathrm{mmol})$ in $\mathrm{Et}_{2} \mathrm{O}(2.5 \mathrm{~mL})$ at $-78^{\circ} \mathrm{C}$ was added dropwise $n$-BuLi $(2.08 \mathrm{M}$ in hexanes, $288 \mu \mathrm{L}, 0.6 \mathrm{mmol})$ via a syringe. After stirring for $30 \mathrm{~min}$, a solution of $(R)-1,2-$ 
epoxybutane ( $87 \mu \mathrm{L}, 72 \mathrm{mg}, 1.0 \mathrm{mmol})$ in $\mathrm{Et}_{2} \mathrm{O} / \mathrm{HMPA}(1.0 \mathrm{~mL}, 10 / 1, \mathrm{v} / \mathrm{v})$ was added dropwise. The resulting mixture was allowed to warm slowly to rt over $1 \mathrm{~h}$ and stirred for an additional $3 \mathrm{~h}$. The reaction was then quenched with $1.0 \mathrm{~N} \mathrm{HCl}(5 \mathrm{~mL})$ and stirred overnight. The reaction mixture was extracted with $\mathrm{Et}_{2} \mathrm{O}(3 \times 10 \mathrm{~mL})$. The combined organic extracts were washed with brine, dried over $\mathrm{MgSO}_{4}$, filtered and concentrated in vacuo. The crude product was purified by flash column chromatography on silica gel (15\% EtOAc/hexanes) to afford three-component adduct 23 as an inseparable mixture of two diastereomers in 1:1 ratio (syn:anti $\geq 20: 1,113 \mathrm{mg}$, $74 \%$ ) as a pale yellow oil. The diastereomeric ratio and the syn to anti ratio were determined by ${ }^{1} \mathrm{H}$ NMR analysis of the crude reaction mixture, and the relative stereochemistry was assigned by analogy to adduct 21. The spectral data were obtained for the mixture of two diastereomers.

${ }^{1} \mathbf{H}$ NMR $\left(500 \mathrm{MHz}, \mathrm{CDCl}_{3}\right) \delta 4.52(\mathrm{dd}, J=4.7,8.5 \mathrm{~Hz}, 1 \mathrm{H}), 4.22(\mathrm{dd}, J=4.5,7.3 \mathrm{~Hz}, 1 \mathrm{H})$, 3.92-3.87 (m, 1H), $3.80(\operatorname{app~q}, J=7.1 \mathrm{~Hz}, 1 \mathrm{H}), 3.11$ (app t, $J=12.9 \mathrm{~Hz}, 1 \mathrm{H}), 3.02-2.82(\mathrm{~m}, 6 \mathrm{H})$, 2.78-2.69 (m, 4H), $2.46(\mathrm{dd}, J=9.4,15.5 \mathrm{~Hz}, 1 \mathrm{H}), 2.30-2.20(\mathrm{~m}, 2 \mathrm{H}), 2.17$ (q, $J=6.9 \mathrm{~Hz}, 1 \mathrm{H})$, 2.13-2.04 (m, 2H), 2.03-1.95 (m, 2H), 1.95-1.81 (m, 2H), 1.61-1.49 (m, 4H), 1.49-1.38 (m, 3H), 1.38-1.21 (m, 10H), 1.19 (d, $J=7.3 \mathrm{~Hz}, 3 \mathrm{H}), 1.01$ (d, $J=7.1 \mathrm{~Hz}, 3 \mathrm{H}), 0.98-0.93$ (m, 6H), 0.92$0.86(\mathrm{~m}, 6 \mathrm{H})$;

${ }^{13}$ C NMR $\left(125 \mathrm{MHz}, \mathrm{CDCl}_{3}\right) \delta 70.9,70.8,69.8,69.7,57.5,57.3,44.4,43.9,42.8,42.4,36.3$, $36.1,31.3,30.8,28.7,28.6,26.6,26.5,26.0,25.7,25.0,24.9,22.8,22.8,14.2,14.2,10.1,10.0$, 8.3, 7.3;

IR (thin film, NaCl) $v_{\max } 3407$ (b), 2931, 2237, 1462, 1422, 1378, 1272, 1131, 986, $909 \mathrm{~cm}^{-1}$;

HRMS (EI, H) $m / z$ calc'd for $\mathrm{C}_{15} \mathrm{H}_{30} \mathrm{O}_{2} \mathrm{~S}_{2} \mathrm{Na}[\mathrm{M}+\mathrm{Na}]^{+} 329.1585$, found 329.1597.
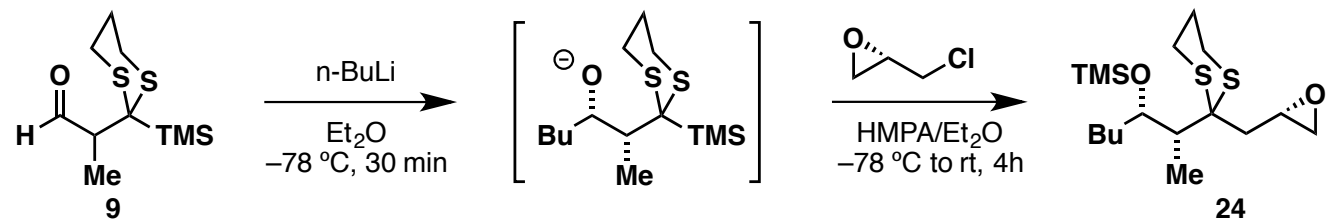

trimethyl((2-(2-(((S)-oxiran-2-yl)methyl)-1,3-dithian-2-yl)heptan-3-yl)oxy)silane (24). To a solution of aldehyde linchpin 9 (124 mg, $0.5 \mathrm{mmol})$ in $\mathrm{Et}_{2} \mathrm{O}(2.5 \mathrm{~mL})$ at $-78{ }^{\circ} \mathrm{C}$ was added dropwise $n$ - $\mathrm{BuLi}(2.08 \mathrm{M}$ in hexanes, $288 \mu \mathrm{L}, 0.6 \mathrm{mmol})$ via a syringe. After stirring for $30 \mathrm{~min}$, a solution of $(S)$-epichlorohydrin $(78.4 \mu \mathrm{L}, 92.5 \mathrm{mg}, 1.0 \mathrm{mmol})$ in $\mathrm{Et}_{2} \mathrm{O} / \mathrm{HMPA}(1.0 \mathrm{~mL}, 10 / 1$, $\mathrm{v} / \mathrm{v}$ ) was added dropwise. The resulting mixture was allowed to warm slowly to rt over $1 \mathrm{~h}$ and 
stirred for an additional $3 \mathrm{~h}$. The reaction was then quenched with saturated aqueous $\mathrm{NH}_{4} \mathrm{Cl}$ solution $(5 \mathrm{~mL})$. The reaction mixture was extracted with $\mathrm{Et}_{2} \mathrm{O}(3 \times 10 \mathrm{~mL})$. The combined organic extracts were washed with brine, dried over $\mathrm{MgSO}_{4}$, filtered and concentrated in vacuo. The crude product was purified by flash column chromatography on silica gel (2\% EtOAc/hexanes with $1 \% \mathrm{Et}_{3} \mathrm{~N}$ ) to afford three-component adduct 24 as an inseparable mixture of two diastereomers in 1:1 ratio (syn:anti $\geq 20: 1,135 \mathrm{mg}, 74 \%$ ) as a colorless oil. The diastereomeric ratio and the syn to anti ratio were determined by ${ }^{1} \mathrm{H}$ NMR analysis of the crude reaction mixture, and the relative stereochemistry was assigned by analogy to adduct $\mathbf{2 1}$. The spectral data were obtained for the mixture of two diastereomers.

${ }^{1} \mathbf{H}$ NMR $\left(500 \mathrm{MHz}, \mathrm{CDCl}_{3}\right) \delta$ 4.48-4.41 (m, 2H), 3.36-3.29 (m, 2H), 2.88-2.67 (m, 10H), 2.51 $(\mathrm{dd}, J=2.8,4.9 \mathrm{~Hz}, 1 \mathrm{H}), 2.48(\mathrm{dd}, J=2.7,4.9 \mathrm{~Hz}, 1 \mathrm{H}), 2.28-2.13(\mathrm{~m}, 4 \mathrm{H}), 2.09-2.03(\mathrm{~m}, 1 \mathrm{H})$, 2.00-1.86 (m, 4H), 1.52-1.42 (m, 4H), 1.39-1.29 (m, 4H), 1.28-1.21 (m, 4H), $1.08(\mathrm{dd}, J=3.9$, $6.7 \mathrm{~Hz}, 6 \mathrm{H}), 0.91(\mathrm{t}, J=7.4 \mathrm{~Hz}, 6 \mathrm{H}), 0.11(\mathrm{~s}, 18 \mathrm{H})$;

${ }^{13} \mathbf{C}$ NMR $\left(125 \mathrm{MHz}, \mathrm{CDCl}_{3}\right) \delta$ 72.1, 72.1, 57.8, 57.7, 49.8, 49.7, 47.7, 47.7, 41.5, 41.3, 39.3, $39.1,37.3,37.3,28.6,28.5,26.3,26.3,26.1,26.1,24.9,24.8,23.1,23.0,14.2,14.2,8.3,8.3,1.2$, 1.2 ;

IR (thin film, NaCl) $v_{\max } 3445$ (br), 2954, 2870, 1714, 1456, 1423, 1379, 1249, 1094, $1041 \mathrm{~cm}^{-1}$; HRMS (EI, H) $m / z$ calc'd for $\mathrm{C}_{17} \mathrm{H}_{34} \mathrm{O}_{2} \mathrm{~S}_{2} \mathrm{SiNa}[\mathrm{M}+\mathrm{Na}]^{+}$385.1662, found 385.1670.
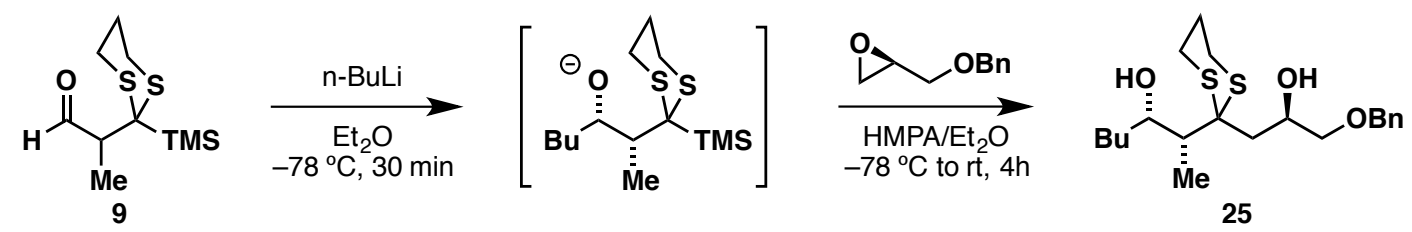

2-(2-((R)-3-(benzyloxy)-2-hydroxypropyl)-1,3-dithian-2-yl)heptan-3-ol (25). To a solution of aldehyde linchpin $9(124 \mathrm{mg}, 0.5 \mathrm{mmol})$ in $\mathrm{Et}_{2} \mathrm{O}(2.5 \mathrm{~mL})$ at $-78^{\circ} \mathrm{C}$ was added dropwise $n$-BuLi $(2.08 \mathrm{M}$ in hexanes, $288 \mu \mathrm{L}, 0.6 \mathrm{mmol})$ via a syringe. After stirring for $30 \mathrm{~min}$, a solution of $(R)$-glycidyl benzyl ether $(152 \mu \mathrm{L}, 164 \mathrm{mg}, 1.0 \mathrm{mmol})$ in $\mathrm{Et}_{2} \mathrm{O} / \mathrm{HMPA}(1.0 \mathrm{~mL}, 10 / 1$, v/v) was added dropwise. The resulting mixture was allowed to warm slowly to $\mathrm{rt}$ over $1 \mathrm{~h}$ and stirred for an additional $3 \mathrm{~h}$. The reaction was then quenched with $1.0 \mathrm{~N} \mathrm{HCl}(5 \mathrm{~mL})$ and stirred overnight. The reaction mixture was extracted with $\mathrm{Et}_{2} \mathrm{O}(3 \times 10 \mathrm{~mL})$. The combined organic extracts were washed with brine, dried over $\mathrm{MgSO}_{4}$, filtered and concentrated in vacuo. The crude product 
was purified by flash column chromatography on silica gel ( $20 \rightarrow 25 \%$ EtOAc/hexanes) to afford three-component adduct $\mathbf{2 5}$ as an inseparable mixture of two diastereomers in 1:1 ratio (syn:anti $\geq 20: 1,135 \mathrm{mg}, 74 \%$ ) as a colorless oil. The diastereomeric ratio and the syn to anti ratio were determined by ${ }^{1} \mathrm{H}$ NMR analysis of the crude reaction mixture, and the relative stereochemistry was assigned by analogy to adduct $\mathbf{2 1}$. The spectral data were obtained for the mixture of two diastereomers.

${ }^{1} \mathbf{H}$ NMR (500 MHz, CDCl $\left.)_{3}\right) \delta .34($ app s, 8H), 7.29 (app s, 2H), 4.57 (d, $J=3.4 \mathrm{~Hz}, 4 \mathrm{H}), 4.50$ (app t, $J=6.2 \mathrm{~Hz}, 1 \mathrm{H}), 4.34$ (app t, $J=6.2 \mathrm{~Hz}, 1 \mathrm{H}$ ), 4.18 (app q, $J=5.9 \mathrm{~Hz}, 1 \mathrm{H}$ ), 4.13 (app q, $J$ $=6.8 \mathrm{~Hz}, 1 \mathrm{H}), 3.50-3.39(\mathrm{~m}, 4 \mathrm{H}), 3.08($ app t, $J=12.2 \mathrm{~Hz}, 3 \mathrm{H}), 2.99-2.90(\mathrm{~m}, 3 \mathrm{H}), 2.81($ app t, $J$ $=12.5 \mathrm{~Hz}, 1 \mathrm{H}), 2.77-2.62(\mathrm{~m}, 4 \mathrm{H}), 2.50(\mathrm{dd}, J=8.8,15.6 \mathrm{~Hz}, 1 \mathrm{H}), 2.36($ app d, $J=15.9 \mathrm{~Hz}, 1 \mathrm{H})$, 2.27-2.14 (m, 3H), 2.10-1.96 (m, 3H), 1.92-1.82 (m, $2 \mathrm{H}), 1.61-1.51(\mathrm{~m}, 2 \mathrm{H}), 1.47-1.23(\mathrm{~m}, 11 \mathrm{H})$, $1.18(\mathrm{~d}, J=7.4 \mathrm{~Hz}, 3 \mathrm{H}), 1.06$ (d, $J=7.4 \mathrm{~Hz}, 3 \mathrm{H}), 0.90$ (app q, $J=6.2 \mathrm{~Hz}, 6 \mathrm{H})$;

${ }^{13} \mathbf{C}$ NMR $\left(125 \mathrm{MHz}, \mathrm{CDCl}_{3}\right) \delta 138.1,138.1,128.6,128.5,127.9,127.9,127.8,127.8,74.6,74.3$, 73.5, 73.4, 70.8, 70.7, 67.8, 67.7, 57.4, 57.3, 44.6, 44.4, 39.8, 39.7, 36.1, 36.1, 28.7, 28.6, 26.5, $26.4,25.9,25.8,25.1,25.0,22.8,22.8,14.2,14.2,8.0,7.5$;

IR (thin film, NaCl) $v_{\max } 3408$ (b), 3029, 2930, 2857, 1495, 1454, 1422, 1273, 1206, $1095 \mathrm{~cm}^{-1}$;

HRMS (EI, H) $m / z$ calc'd for $\mathrm{C}_{21} \mathrm{H}_{34} \mathrm{O}_{3} \mathrm{~S}_{2} \mathrm{Na}[\mathrm{M}+\mathrm{Na}]^{+} 421.1847$, found 421.1846 . 


\section{Synthesis of enantiomerically enriched aldehyde bifunctional linchpin $(+)-9$ :}

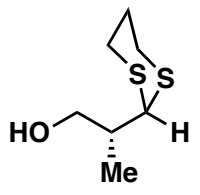

(-)-16

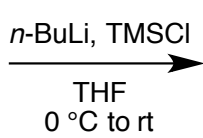

(87\%)

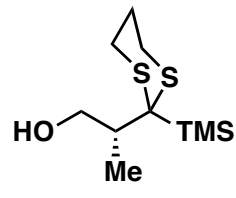

(-)-S-1

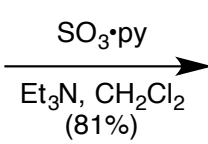

Alcohol (-)-16 was prepared from commercially available $(-)-(R)$-Roche ester according to literature procedures. ${ }^{2}$

(-)-(R)-2-(2-(trimethylsilyl)-1,3-dithian-2-yl)propan-1-ol [(-)-S-1]. To a precooled $\left(0{ }^{\circ} \mathrm{C}\right)$ solution of (-)-14 (2.79 g, $15.65 \mathrm{mmol})$ in THF $(31 \mathrm{~mL})$ was added dropwise $n$-BuLi $(2.3 \mathrm{M}$ solution in hexane, $14.97 \mathrm{~mL}, 34.4 \mathrm{mmol})$. The resulting red solution was stirred at $0{ }^{\circ} \mathrm{C}$ for 30 min, then TMSCl ( $4.40 \mathrm{ml}, 34.4 \mathrm{mmol}$ ) was added dropwise. The reaction mixture rapidly turned pale yellow. The ice bath was then removed and the reaction was allowed to warm to $\mathrm{rt}$ and stirred for $1.5 \mathrm{~h}$, before quenching with $1 \mathrm{~N}$ aq. $\mathrm{HCl}(20 \mathrm{~mL})$ and stirred for an additional $30 \mathrm{~min}$. the reaction mixture was extracted with EtOAc $(3 \times 40 \mathrm{~mL})$. The combined organic extracts were washed with brine, dried over $\mathrm{Na}_{2} \mathrm{SO}_{4}$, filtered and concentrated in vacuo. The crude product was purified by flash column chromatography on silica gel $(20 \%$ EtOAc/hexanes) to afford (-)-S-1 (3.20 g, 82\%) as a white solid. Data consistent with racemic S-1 (vide supra). $[\boldsymbol{\alpha}]_{\mathbf{D}}{ }^{20}=-10.7\left(\mathrm{c} 1.12, \mathrm{CHCl}_{3}\right)$.

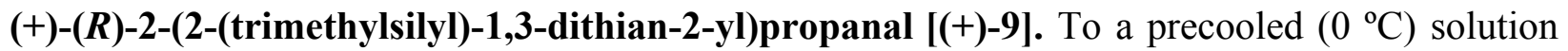
of (-)-S-1 (1.668 g, $6.66 \mathrm{mmol})$ in $\mathrm{CH}_{2} \mathrm{Cl}_{2}(33 \mathrm{~mL})$ and DMSO $(2.36 \mathrm{~mL}, 33.3 \mathrm{mmol})$, were added $\mathrm{Et}_{3} \mathrm{~N}$ (4.64 mL, $33.3 \mathrm{mmol}$ ) and $\mathrm{SO}_{3} \bullet$ pyridine $(3.18 \mathrm{~g}, 20.0 \mathrm{mmol})$. The ice/water bath was then removed and the reaction stirred at $\mathrm{rt}$ for $2.5 \mathrm{~h}$, before quenching with aqueous $\mathrm{pH} 7$ buffer. $\mathrm{CH}_{2} \mathrm{Cl}_{2}(50 \mathrm{~mL})$ was added, the layers separated, and the organic layer washed with sat. aq. $\mathrm{CuSO}_{4}(3 \times 15 \mathrm{~mL})$ and brine $(15 \mathrm{~mL})$, dried over $\mathrm{Na}_{2} \mathrm{SO}_{4}$, filtered, and concentrated in vacuo. The residue was purified by flash column chromatography (10\% EtOAc/hexanes) to afford (+)-9 as a white solid (1.345 g, 81\%). Data consistent with racemic 9 (vide supra). $[\alpha]_{\mathbf{D}}^{22}=+52.7\left(\mathrm{c} 0.47, \mathrm{CHCl}_{3}\right)$.

(-)-9: m.p. $35-37^{\circ} \mathrm{C} ;[\alpha]_{\mathbf{D}}{ }^{22}=-58.5\left(\mathrm{c} 1.05, \mathrm{CH}_{2} \mathrm{Cl}_{2}\right)$. 
Determination of enantiomeric excess by chromatography on chiral stationary phase:

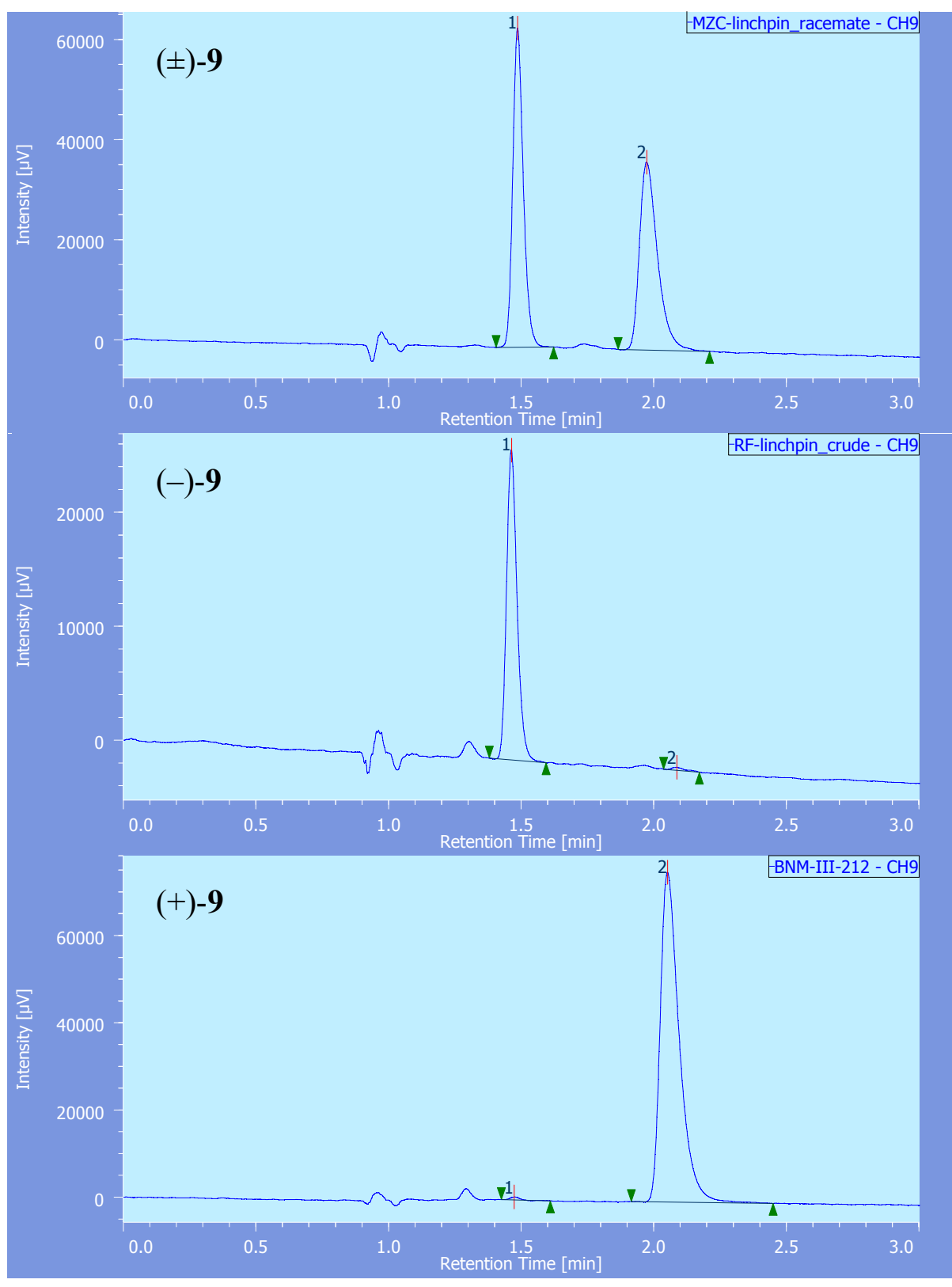

\begin{tabular}{ccccc} 
Sample & $\mathbf{t}_{\mathrm{R}} \mathbf{1}(\mathbf{m i n})$ & Area $\mathbf{1}(\mathbf{\%})$ & $\mathbf{t}_{\mathrm{R}} \mathbf{2}(\mathbf{m i n})$ & Area $\mathbf{2}(\%)$ \\
\hline $\mathbf{( \pm ) - 9}$ & 1.487 & 49.79 & 1.973 & 50.21 \\
$(-)-9$ & 1.463 & 98.83 & 2.087 & 1.17 \\
$(+)-9$ & 1.473 & 0.48 & 2.052 & 99.52
\end{tabular}

Conditions: column: CHIRALPAK AD-H; eluent: 8:2 supercritical $\mathrm{CO}_{2} / \mathrm{MeOH}$; flow rate: $4 \mathrm{~mL} / \mathrm{min}$; detection wavelength: $220 \mathrm{~nm}$. 


\section{Determination of diastereoselectivity of addition to linchpin 9:}

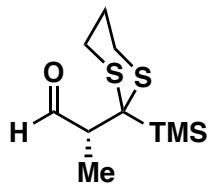

$(+)-9$

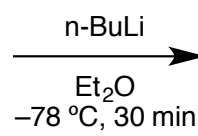

$-78^{\circ} \mathrm{C}, 30 \mathrm{~min}$

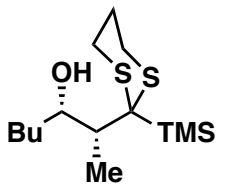

$(+)-S-2$

(+)-(2R,3S)-2-(2-(trimethylsilyl)-1,3-dithian-2-yl)heptan-3-ol [(+)-S-2]

To a precooled $\left(-78{ }^{\circ} \mathrm{C}\right)$ solution of $(+)-9(50 \mathrm{mg}, 0.201 \mathrm{mmol})$ in $\mathrm{Et}_{2} \mathrm{O}(1.0 \mathrm{~mL})$ was added n$\operatorname{BuLi}(97 \mu 1,2.5 \mathrm{M}$ solution in hexanes, $0.241 \mathrm{mmol})$. The solution was stirred at $-78{ }^{\circ} \mathrm{C}$ for 30 min. Sat. aq. $\mathrm{NH}_{4} \mathrm{Cl}(1 \mathrm{~mL})$ was then added and the resulting biphasic mixture was extracted with $\mathrm{Et}_{2} \mathrm{O}$. The combined organic layers were dried over $\mathrm{Na}_{2} \mathrm{SO}_{4}$, filtered and concentrated in vacuo. ${ }^{1} \mathrm{H}$ NMR analysis of the crude reaction mixture shows the presence of only one diastereomer of the desired product $(d r>20: 1)$. The crude residue was then purified by column chromatography (5\% EtOAc/hexanes) to furnish S-2 (43 mg, 70\%) as a clear colorless oil. $[\alpha]_{\mathbf{D}}{ }^{22}=+20.6\left(\mathrm{c} 0.27, \mathrm{CH}_{2} \mathrm{Cl}_{2}\right)$;

${ }^{1}$ H NMR (500 MHz, $\left.\mathrm{CDCl}_{3}\right) \delta 4.32$ - 4.17 (m, $\left.1 \mathrm{H}\right), 3.04$ - 2.86 (m, $\left.2 \mathrm{H}\right), 2.54$ (qd, J = 4.2, 13.3 Hz, 2 H), 2.37 - 2.20 (m, 2 H), 2.14 - 2.01 (m, 1 H), 1.95 - 1.82 (m, 1 H), 1.69 - 1.50 (m, 1 H), 1.49 - 1.29 (m, $5 \mathrm{H}), 1.22$ (d, $J=7.1 \mathrm{~Hz}, 3 \mathrm{H}), 0.93$ (t, $J=7.0 \mathrm{~Hz}, 3 \mathrm{H}), 0.27$ (s, $9 \mathrm{H})$; ${ }^{13}$ C NMR (125 MHz, $\left.\mathrm{CDCl}_{3}\right) \delta 71.9,46.3,42.3,36.7,28.7,25.5,25.1,24.2,22.9,14.2,11.4$, 0.5

IR (thin film, KBr) $v_{\max } 3414,2932,1681,1441,1138 \mathrm{~cm}^{-1}$;

HRMS (EI, H) $m / z$ calc'd for $\mathrm{C}_{14} \mathrm{H}_{30} \mathrm{ONaSiS}_{2}[\mathrm{M}+\mathrm{Na}]^{+}$329.1402, found 329.1405.

The Trost-Mosher esters of S-2 were prepared and analyzed according to literature procedures: ${ }^{4}$

To a solution of $\mathbf{S - 2}(12.3 \mathrm{mg}, 0.040 \mathrm{mmol})$ in $\mathrm{CH}_{2} \mathrm{Cl}_{2}(0.7 \mathrm{~mL})$ was added either $(R)-\mathrm{MTPA}-\mathrm{OH}$ or $(S)$-MTPA-OH (29 mg, $0.124 \mathrm{mmol})$, followed by DCC (26 mg, $0.124 \mathrm{mmol})$ and DMAP (15 $\mathrm{mg}, 0.124 \mathrm{mmol})$. A white precipitate formed within one minute. The resulting reaction mixture was stirred overnight at $\mathrm{rt}$, then filtered through cotton and concentrated in vacuo. The crude residue was chromatographed (5\% EtOAc/hexanes) to furnish samples of $\mathbf{S - 3}$ and $\mathbf{S - 4}$, analyzed by ${ }^{1} \mathrm{H}$ NMR. 


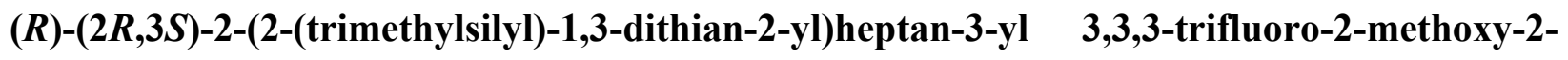
phenylpropanoate (S-3)

${ }^{1}$ H NMR (500 MHz, $\left.\mathrm{CDCl}_{3}\right) \delta 7.70-7.63(\mathrm{~m}, 2 \mathrm{H}), 7.40-7.35$ (m, $\left.3 \mathrm{H}\right), 5.60$ (dd, $J=4.8,7.1$ Hz, 1 H), 3.65 - 3.62 (m, 3 H), 2.78 - 2.63 (m, 2 H), 2.49 - 2.38 (m, 2 H), 2.34 (ddd, $J=3.5,5.9$, $14.0 \mathrm{~Hz}, 1 \mathrm{H}), 1.99$ - 1.89 (m, $1 \mathrm{H}), 1.82$ (s, $2 \mathrm{H}), 1.71$ - 1.61 (m, 1 H), 1.44 - 1.32 (m, 4 H), 1.28 $(\mathrm{d}, J=7.1 \mathrm{~Hz}, 3 \mathrm{H}), 0.91(\mathrm{t}, J=7.1 \mathrm{~Hz}, 3 \mathrm{H}), 0.15(\mathrm{~s}, 9 \mathrm{H})$.

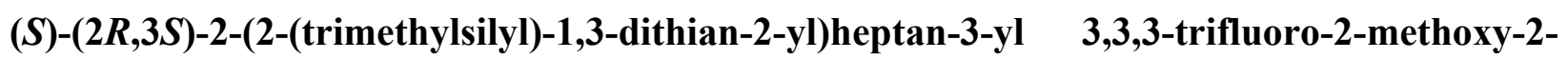
phenylpropanoate (S-4)

${ }^{1} \mathbf{H}$ NMR $\left(500 \mathrm{MHz}, \mathrm{CDCl}_{3}\right) \delta 7.81-7.52(\mathrm{~m}, 2 \mathrm{H}), 7.49-7.29(\mathrm{~m}, 3 \mathrm{H}), 5.71(\mathrm{t}, J=6.0 \mathrm{~Hz}, 1$ H), 3.54 (s, $3 \mathrm{H}), 2.76$ (dtd, $J=3.3,10.3,13.8 \mathrm{~Hz}, 2 \mathrm{H}), 2.57$ (ddd, $J=3.6,6.5,13.9 \mathrm{~Hz}, 1 \mathrm{H}$ ), 2.50 - 2.39 (m, $1 \mathrm{H}), 2.34$ (q, $J=7.3 \mathrm{~Hz}, 1 \mathrm{H}), 2.01$ (tdd, J = 3.1, 6.3, $13.2 \mathrm{~Hz}, 1 \mathrm{H}), 1.90-1.79$ (m, $1 \mathrm{H}), 1.70-1.59(\mathrm{~m}, 2 \mathrm{H}), 1.41-1.23(\mathrm{~m}, 4 \mathrm{H}), 1.21(\mathrm{~d}, \mathrm{~J}=7.1 \mathrm{~Hz}, 3 \mathrm{H}), 0.89(\mathrm{t}, J=7.1 \mathrm{~Hz}$, $3 \mathrm{H}), 0.23$ (s, $9 \mathrm{H})$.

\begin{tabular}{|c|c|c|c|}
\hline $\begin{array}{l}\text { Nucleus or } \\
\text { Nuclei }\end{array}$ & $\begin{array}{c}\text { (R)-Ester (S-3) } \\
\delta_{\mathrm{s}-3}(\mathrm{ppm})\end{array}$ & $\begin{array}{c}\text { (S)-Ester (S-4) } \\
\delta_{\mathrm{S}-4}(\mathrm{ppm})\end{array}$ & $\delta_{\mathrm{S}-3}-\delta_{\mathrm{S}-4}(p p m)$ \\
\hline $\mathrm{m}$ & 3.63 & 3.54 & +0.09 \\
\hline e & 2.42 & 2.34 & +0.08 \\
\hline $\mathrm{f}$ & 1.28 & 1.21 & +0.07 \\
\hline$b, b^{\prime}$ & 1.36 & 1.3 & +0.06 \\
\hline $\mathrm{c}$ & 1.66 & 1.63 & +0.03 \\
\hline a & 0.91 & 0.89 & +0.02 \\
\hline $\mathrm{n}$ & 7.64 & 7.64 & 0 \\
\hline $\mathrm{i}$ & 2.42 & 2.44 & -0.02 \\
\hline$n^{\prime}$ & 7.38 & 7.41 & -0.03 \\
\hline $\mathrm{h}$ & 2.7 & 2.76 & -0.06 \\
\hline 1 & 1.79 & 1.85 & -0.06 \\
\hline $\mathrm{k}$ & 1.94 & 2.01 & -0.07 \\
\hline$g$ & 0.15 & 0.23 & -0.08 \\
\hline$d$ & 5.6 & 5.71 & -0.11 \\
\hline j & 2.34 & 2.57 & -0.23 \\
\hline
\end{tabular}
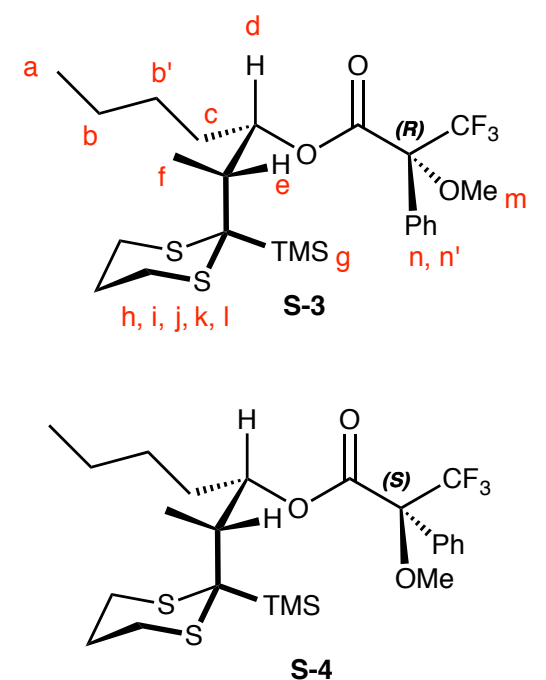


\section{Application to the synthesis of C16-C29 fragment of rhizopodin}

Synthesis of vinyliodide 25:

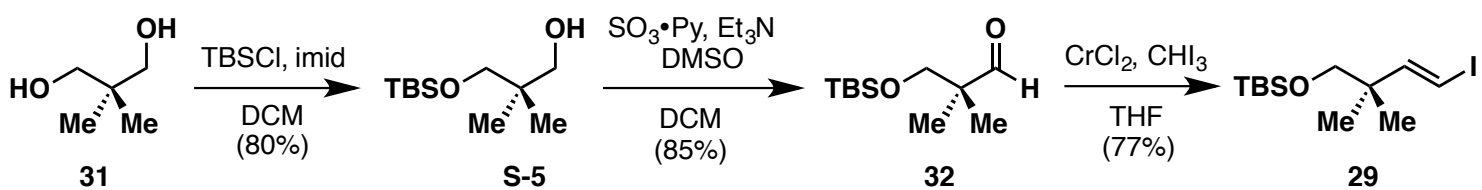

3-((tert-butyldimethylsilyl)oxy)-2,2-dimethylpropanal (32) was prepared from 2,2-dimethyl1,3-propanediol in two steps according to literature procedures. ${ }^{5}$

(E)-tert-butyl((4-iodo-2,2-dimethylbut-3-en-1-yl)oxy)dimethylsilane (29) via Takai-Utimoto olefination: ${ }^{6}$ To a suspension of $\mathrm{CrCl}_{2}(2.77 \mathrm{~g}, 22.5 \mathrm{mmol})$ in THF $(35 \mathrm{~mL})$ at $0{ }^{\circ} \mathrm{C}$ was added a solution of aldehyde $27(810 \mathrm{mg}, 3.75 \mathrm{mmol})$ and $\mathrm{CHI}_{3}(3.0 \mathrm{~g}, 7.5 \mathrm{mmol})$ in THF $(10 \mathrm{~mL})$. The resulting mixture was stirred in the dark at $0{ }^{\circ} \mathrm{C}$ for $1 \mathrm{~h}$, and then warmed to $\mathrm{rt}$ overnight. The reaction mixture was quenched with saturated aqueous $\mathrm{Na}_{2} \mathrm{~S}_{2} \mathrm{O}_{3}$ solution $(30 \mathrm{~mL})$ and extracted with $\mathrm{Et}_{2} \mathrm{O}(3 \times 30 \mathrm{~mL})$. The combined organic extracts were washed with additional $\mathrm{Na}_{2} \mathrm{~S}_{2} \mathrm{O}_{3}$ solution $(3 \times 30 \mathrm{~mL})$ and brine, dried over $\mathrm{MgSO}_{4}$, filtered and concentrated in vacuo. The crude reaction mixture was taken up in hexanes to precipitate out excess $\mathrm{CHI}_{3}$, filtered and concentrated in vacuo. This process was repeated three times. The crude product was purified by flash column chromatography on silica gel (100\% hexanes) to afford vinyliodide 25 (980 mg, $77 \%)$ as a colorless oil.

${ }^{1} \mathbf{H}$ NMR $\left(500 \mathrm{MHz}, \mathrm{CDCl}_{3}\right) \delta 6.56(\mathrm{~d}, J=15.2 \mathrm{~Hz}, 1 \mathrm{H}), 6.01(\mathrm{~d}, J=15.2 \mathrm{~Hz}, 1 \mathrm{H}), 3.29(\mathrm{~s}, 2 \mathrm{H})$, $0.98(\mathrm{~s}, 6 \mathrm{H}), 0.89(\mathrm{~s}, 9 \mathrm{H}), 0.03(\mathrm{~s}, 6 \mathrm{H})$;

${ }^{13}$ C NMR $\left(125 \mathrm{MHz}, \mathrm{CDCl}_{3}\right) \delta 153.6,73.9,71.2,43.2,26.0,23.3,18.4,-5.3$;

IR (thin film, $\mathrm{NaCl}$ ) $v_{\max } 2956,2929,2856,1731,1600,1471,1390,1361,1256,1102 \mathrm{~cm}^{-1}$;

HRMS (EI, H) $m / z$ calc'd for $\mathrm{C}_{11} \mathrm{H}_{22} \mathrm{IOSi}\left[\mathrm{M}-\mathrm{CH}_{3}\right]^{+} 325.0479$, found 325.0488. 


\section{Synthesis of epoxide 30:}

a. Mixture of diastereomers $\left(30+30^{\prime}\right)$ :

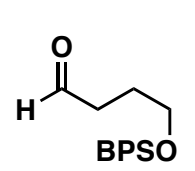

33

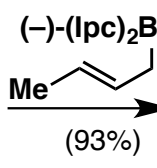

$93 \%)$

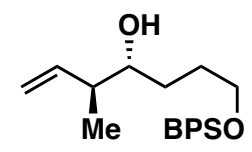

$(-)-34$

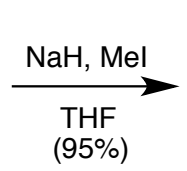

$(95 \%)$

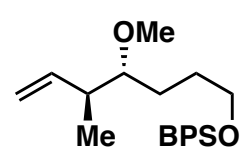

$(-)-S-6$

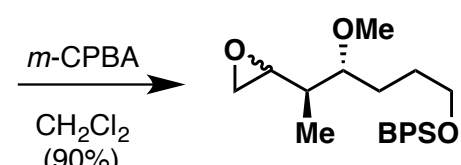

$30+30^{\prime}$

Enantiomerically enriched homoallylic ether (-)-S-6 was prepared from TBDPS-protected aldehyde $\mathbf{3 3}$ in two steps following the literature procedures. ${ }^{7}$

Epoxide 30. To a solution of ether (-)-S-6 $(900 \mathrm{mg}, 2.27 \mathrm{mmol})$ in $\mathrm{CH}_{2} \mathrm{Cl}_{2}(9 \mathrm{~mL})$ at $0{ }^{\circ} \mathrm{C}$ was added portionwise $m$-CPBA $(\leq 77 \%, 764 \mathrm{mg}, 3.40 \mathrm{mmol})$. The resulting clear solution was warmed to $\mathrm{rt}$ and stirred overnight. The reaction mixture was quenched slowly with saturated aqueous $\mathrm{Na}_{2} \mathrm{~S}_{2} \mathrm{O}_{3}$ solution $(10 \mathrm{~mL})$, and extracted with $\mathrm{CH}_{2} \mathrm{Cl}_{2}(3 \times 30 \mathrm{~mL})$. The combined organic extracts were washed with saturated aqueous $\mathrm{NaHCO}_{3}$ solution and brine, dried over $\mathrm{MgSO}_{4}$, filtered and concentrated in vacuo. The crude product was purified by flash column chromatography on silica gel (5\% EtOAc/hexanes) to afford epoxide 30 (1:1 mixture of diastereomers, 30+30', $824 \mathrm{mg}, 90 \%$ ) as a colorless oil. Analytical samples of each of the epoxide diastereomers were obtained by chromatographic purification and stereochemistry was assigned by comparison with sample from diastereoselective synthesis (vide infra).

tert-butyl(((4R,5R)-4-methoxy-5-((R)-oxiran-2-yl)hexyl)oxy)diphenylsilane $[(-)-30]$

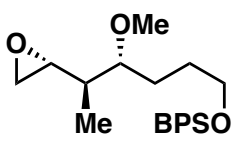

$[\alpha]_{\mathbf{D}}{ }^{22}=-5.3\left(\mathrm{c} 0.44, \mathrm{CH}_{2} \mathrm{Cl}_{2}\right)$; 3.34 (s, 3H), 3.24-3.19 (m, 1H), 2.90-2.85 (m, 1H), 2.72 (app t, $J=4.2 \mathrm{~Hz}, 1 \mathrm{H}), 2.48-2.45$ (m, $1 \mathrm{H}), 1.76-1.54(\mathrm{~m}, 5 \mathrm{H}), 1.06(\mathrm{~s}, 9 \mathrm{H}), 0.90(\mathrm{~d}, J=7.2 \mathrm{~Hz}, 3 \mathrm{H})$;

${ }^{13} \mathbf{C}$ NMR $\left(125 \mathrm{MHz}, \mathrm{CDCl}_{3}\right) \delta 135.7,134.2,129.7,127.7,83.3,64.1,57.7,53.7,45.4,38.6$, 28.8, 27.0, 26.9, 19.3, 11.4;

IR (thin film, $\mathrm{NaCl}$ ) $v_{\max } 3071,3047,2931,2857,2822,1472,1428,1388,1360,1110 \mathrm{~cm}^{-1}$; HRMS (EI, H) $m / z$ calc'd for $\mathrm{C}_{25} \mathrm{H}_{36} \mathrm{O}_{3} \mathrm{SiNa}[\mathrm{M}+\mathrm{Na}]^{+} 435.2326$, found 435.2324. 
tert-butyl(((4R,5R)-4-methoxy-5-((S)-oxiran-2-yl)hexyl)oxy)diphenylsilane

(-)-30'

$[\alpha]_{\mathbf{D}}{ }^{22}=-7.6\left(\mathrm{c} 1.0, \mathrm{CH}_{2} \mathrm{Cl}_{2}\right)$;

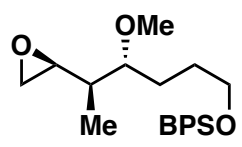

$(-)-30$

${ }^{1} \mathbf{H}$ NMR $\left(500 \mathrm{MHz}, \mathrm{CDCl}_{3}\right) \delta 7.67(\mathrm{~d}, J=7.1 \mathrm{~Hz}, 4 \mathrm{H}), 7.45-7.35(\mathrm{~m}, 6 \mathrm{H}), 3.72-3.64(\mathrm{~m}, 2 \mathrm{H})$, $3.33(\mathrm{~s}, 3 \mathrm{H}), 3.15-3.10(\mathrm{~m}, 1 \mathrm{H}), 2.86-2.82(\mathrm{~m}, 1 \mathrm{H}), 2.78(\mathrm{t}, J=4.4 \mathrm{~Hz}, 1 \mathrm{H}), 2.59-2.57(\mathrm{~m}, 1 \mathrm{H})$, 1.69-1.44 (m, 5H), 1.05 (s, 9H), $1.00(\mathrm{~d}, J=7.1 \mathrm{~Hz}, 3 \mathrm{H})$;

${ }^{13} \mathbf{C}$ NMR $\left(125 \mathrm{MHz}, \mathrm{CDCl}_{3}\right) \delta 135.7,134.1,129.7,127.8,83.5,64.0,58.0,54.7,47.5,39.6$, 28.3, 27.4, 27.0, 19.4, 12.9;

IR (thin film, NaCl) $v_{\max } 3070,3047,2930,2857,1472,1428,1388,1360,1109,998 \mathrm{~cm}^{-1}$; HRMS (EI, H) $m / z$ calc'd for $\mathrm{C}_{25} \mathrm{H}_{36} \mathrm{O}_{3} \mathrm{SiNa}[\mathrm{M}+\mathrm{Na}]^{+} 435.2326$, found 435.2324.

\section{b. Diastereoselective synthesis:}

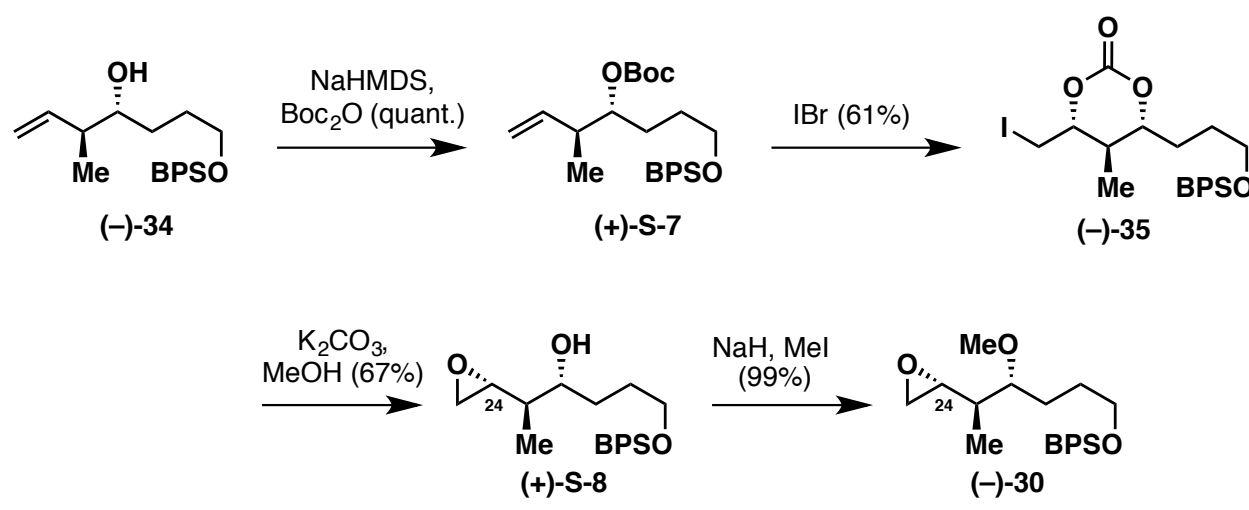

tert-butyl ((3S,4R)-7-((tert-butyldiphenylsilyl)oxy)-3-methylhept-1-en-4-yl) carbonate [(+)S-7] To a precooled $\left({ }^{\circ} \mathrm{C}\right)$ solution of $(-)-34(1.26 \mathrm{~g}, 3.19 \mathrm{mmol})$ in THF $(16 \mathrm{ml})$ was added dropwise NaHMDS (1.0 M solution in THF, $4.78 \mathrm{ml}, 4.78 \mathrm{mmol})$. The reaction was stirred for $30 \mathrm{~min}$ at $0{ }^{\circ} \mathrm{C}$, then $\mathrm{Boc}_{2} \mathrm{O}(1.04 \mathrm{~g}, 4.78 \mathrm{mmol})$ was added and the reaction was allowed to warm to $\mathrm{rt}$ and stirred for $15 \mathrm{~min}$. Brine $(5 \mathrm{~mL})$ was added and the resulting mixture was extracted with $\mathrm{Et}_{2} \mathrm{O}(3 \times 20 \mathrm{~mL})$. The organic layers were combined and dried over $\mathrm{Na}_{2} \mathrm{SO}_{4}$, filtered and concentrated in vacuo. The residue purified by flash column chromatography $\left(20 \% \mathrm{Et}_{2} \mathrm{O} / \mathrm{h} \mathrm{xane}\right)$ to afford S-7 as a clear colorless oil (1.70 g, quant.).

$[\alpha]_{\mathbf{D}}{ }^{22}=+2.9\left(\mathrm{c} 0.92, \mathrm{CH}_{2} \mathrm{Cl}_{2}\right)$;

${ }^{1}$ H NMR $\left(500 \mathrm{MHz}, \mathrm{CDCl}_{3}\right) \delta 7.67(\mathrm{~d}, J=7.3 \mathrm{~Hz}, 4 \mathrm{H}), 7.46$ - 7.36 (m, $\left.6 \mathrm{H}\right), 5.77$ (ddd, $J=8.1$, 10.3, 18.4 Hz, $1 \mathrm{H}), 5.11$ - $5.02(\mathrm{~m}, 2 \mathrm{H}), 4.66$ - 4.60 (m, $1 \mathrm{H}), 3.73$ - 3.62 (m, $2 \mathrm{H}), 2.43$ (sxt, $J=$ $6.8 \mathrm{~Hz}, 1 \mathrm{H}), 1.74$ - 1.51 (m, $4 \mathrm{H}), 1.48(\mathrm{~s}, 9 \mathrm{H}), 1.09$ - 1.01 (m, $12 \mathrm{H})$; 
${ }^{13}$ C NMR (125 MHz, $\left.\mathrm{CDCl}_{3}\right) \delta 153.9,139.6,135.7,134.1,129.7,127.7,115.7,81.7,80.1,63.6$, 41.9, 28.7, 28.0, 27.9, 27.6, 27.0, 19.4, 16.0;

IR (thin film, KBr) $v_{\max } 3454,2931,1737,1368,1278,1166,1111 \mathrm{~cm}^{-1}$;

HRMS (EI, H) $m / z$ calc'd for $\mathrm{C}_{29} \mathrm{H}_{42} \mathrm{O}_{4} \mathrm{NaSi}[\mathrm{M}+\mathrm{Na}]^{+}$505.2750, found 505.2755.

\section{(4R,5R,6R)-4-(3-((tert-butyldiphenylsilyl)oxy)propyl)-6-(iodomethyl)-5-methyl-1,3-dioxan-}

\section{2-one [(-)-35]}

To a precooled $\left(-85^{\circ} \mathrm{C}, \mathrm{Et}_{2} \mathrm{O} /\right.$ dry ice bath) solution of $(+)-\mathrm{S}-7(199 \mathrm{mg}, 0.400 \mathrm{mmol})$ in $\mathrm{CH}_{2} \mathrm{Cl}_{2}$ $(4.0 \mathrm{~mL})$ was added $\mathrm{IBr}(124 \mathrm{mg}, 0.600 \mathrm{mmol})$. The resulting reaction mixture was stirred at -85 ${ }^{\circ} \mathrm{C}$ for $30 \mathrm{~min}$, then warmed to $0{ }^{\circ} \mathrm{C}$. Were then added sat. aq. $\mathrm{Na}_{2} \mathrm{~S}_{2} \mathrm{O}_{3}(1 \mathrm{~mL})$, sat. aq. $\mathrm{NaHCO}_{3}$ $(1 \mathrm{~mL})$. Layers were partitioned and the aqueous layer was extracted with $\mathrm{Et}_{2} \mathrm{O}(3 \times 5 \mathrm{~mL})$, dried over $\mathrm{Na}_{2} \mathrm{SO}_{4}$, filtered and concentrated in vacuo. ${ }^{1} \mathrm{H} \mathrm{NMR}$ analysis of the crude reaction mixture revealed a diastereomeric ratio of 11:1. Flash column chromatography $(15 \% \rightarrow 50 \%$ EtOAc/hexane) afforded 35 (single diastereomer) as a clear pale yellow oil (135 mg, 61\%). $[\alpha]_{\mathbf{D}}{ }^{22}=-0.1\left(\mathrm{c} 2.0, \mathrm{CH}_{2} \mathrm{Cl}_{2}\right)$;

${ }^{1}$ H NMR $\left(500 \mathrm{MHz}, \mathrm{CDCl}_{3}\right) \delta 7.72$ - $7.62(\mathrm{~m}, 4 \mathrm{H}), 7.48$ - $7.37(\mathrm{~m}, 6 \mathrm{H}), 4.23$ - $4.16(\mathrm{~m}, 1 \mathrm{H})$, 3.81 - 3.69 (m, 3 H), 3.57 (dd, J = 2.9, $11.6 \mathrm{~Hz}, 1 \mathrm{H}), 3.37$ (dd, J = 4.0, $11.7 \mathrm{~Hz}, 1 \mathrm{H}), 2.04$ - 1.91 (m, $2 \mathrm{H}), 1.90$ - $1.79(\mathrm{~m}, 1 \mathrm{H}), 1.75$ - 1.61 (m, $2 \mathrm{H}), 1.07$ (s, $9 \mathrm{H}), 0.97$ (d, J = 6.7 Hz, $3 \mathrm{H})$;

${ }^{13}$ C NMR $\left(125 \mathrm{MHz}, \mathrm{CDCl}_{3}\right) \delta 148.7,135.6,133.8,133.8,129.8,127.8,82.4,80.4,63.1,37.0$, 28.9, 27.0, 19.3, 12.1, 6.6;

IR (thin film, KBr) $v_{\max } 2929,2854,1755,1427,1387,1223,1187,1111 \mathrm{~cm}^{-1}$;

HRMS (EI, H) $m / z$ calc'd for $\mathrm{C}_{25} \mathrm{H}_{33} \mathrm{O}_{4} \mathrm{NaSiI}[\mathrm{M}+\mathrm{Na}]^{+}$575.1091, found 575.1096.

Cis-stereochemistry assigned by comparison of NMR spectra with minor diastereomer ${ }^{8}$ (analytical sample obtained):

(4R,5R,6S)-4-(3-((tert-butyldiphenylsilyl)oxy)propyl)-6-(iodomethyl)-5methyl-1,3-dioxan-2-one [(+)-35']

$[\boldsymbol{\alpha}]_{\mathbf{D}}{ }^{22}=+31.7\left(\mathrm{c} 1.0, \mathrm{CH}_{2} \mathrm{Cl}_{2}\right)$;

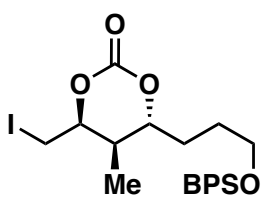

$(+)-35$

${ }^{1}$ H NMR $\left(500 \mathrm{MHz}, \mathrm{CDCl}_{3}\right) \delta 7.66(\mathrm{~d}, J=6.7 \mathrm{~Hz}, 4 \mathrm{H}), 7.48$ - $7.38(\mathrm{~m}, 6 \mathrm{H}), 4.63$ (ddd, $J=3.4$, 6.3, $9.5 \mathrm{~Hz}, 1 \mathrm{H}), 4.31$ - $4.25(\mathrm{~m}, 1 \mathrm{H}), 3.79$ - $3.69(\mathrm{~m}, 2 \mathrm{H}), 3.35(\mathrm{dd}, J=6.3,10.3 \mathrm{~Hz}, 1 \mathrm{H})$, 
$3.17(\mathrm{dd}, J=8.6,10.2 \mathrm{~Hz}, 1 \mathrm{H}), 2.35-2.27(\mathrm{~m}, 1 \mathrm{H}), 1.90-1.72(\mathrm{~m}, 3 \mathrm{H}), 1.72-1.61(\mathrm{~m}, 1 \mathrm{H})$, 1.09 - $1.04(\mathrm{~m}, 12 \mathrm{H})$;

${ }^{13}$ C NMR $\left(125 \mathrm{MHz}, \mathrm{CDCl}_{3}\right) \delta 148.0,135.7,133.7,129.9,127.9,84.1,77.9,63.0,32.0,30.9$, 28.0, 27.0, 19.3, 11.0, 0.7;

IR (thin film, KBr) $v_{\max } 2929,2854,1751,1427,1387,1174,1111 \mathrm{~cm}^{-1}$;

HRMS (EI, H) $m / z$ calc'd for $\mathrm{C}_{25} \mathrm{H}_{33} \mathrm{O}_{4} \mathrm{NaSiI}[\mathrm{M}+\mathrm{Na}]^{+}$575.1091, found 575.1091.

(2R,3R)-6-((tert-butyldiphenylsilyl)oxy)-2-((R)-oxiran-2-yl)hexan-3-ol $\quad[(+)-S-8] . \quad$ To a solution of $(-)-36(1.26 \mathrm{~g}, 2.030 \mathrm{mmol})$ in $\mathrm{MeOH}(20 \mathrm{~mL})$ was added $\mathrm{K}_{2} \mathrm{CO}_{3}(0.842 \mathrm{~g}, 6.09$ mmol) and the resulting mixture was stirred at rt overnight. Sat. aq. $\mathrm{NaHCO}_{3}(3 \mathrm{~mL})$ was then added, followed by water $(3 \mathrm{~mL})$ and sat. aq. $\mathrm{Na}_{2} \mathrm{~S}_{2} \mathrm{O}_{3}(3 \mathrm{~mL})$. The mixture was extracted with EtOAc $(3 \times 20 \mathrm{~mL})$. The organic layers were combined, dried over $\mathrm{Na}_{2} \mathrm{SO}_{4}$, filtered and concentrated. The residue was purified by flash column chromatography (15\% EtOAc/hexane) to afford S-8 as a clear colorless oil (563 mg, 67\%).

$[\alpha]_{\mathbf{D}}{ }^{22}=+5.66\left(\mathrm{c} 1.0, \mathrm{CH}_{2} \mathrm{Cl}_{2}\right)$;

${ }^{1} \mathbf{H}$ NMR $\left(500 \mathrm{MHz}, \mathrm{CDCl}_{3}\right) \delta 7.72$ - $7.66(\mathrm{~m}, 4 \mathrm{H}), 7.48$ - $7.37(\mathrm{~m}, 6 \mathrm{H}), 3.76$ - $3.68(\mathrm{~m}, 3 \mathrm{H})$, 2.96 (s, $1 \mathrm{H}), 2.77$ (t, $J=4.5 \mathrm{~Hz}, 1 \mathrm{H}), 2.73$ (br. s., $1 \mathrm{H}$ ), 2.50 (dd, $J=2.8,5.0 \mathrm{~Hz}, 1 \mathrm{H}$ ), 1.80 $1.66(\mathrm{~m}, 3 \mathrm{H}), 1.60-1.51$ (m, $1 \mathrm{H}), 1.37$ (sxt, $J=7.1 \mathrm{~Hz}, 1 \mathrm{H}), 1.07$ (s, $9 \mathrm{H}), 0.98$ (d, $J=6.9 \mathrm{~Hz}$, $3 \mathrm{H})$;

${ }^{13} \mathbf{C}$ NMR $\left(125 \mathrm{MHz}, \mathrm{CDCl}_{3}\right) \delta 135.7,133.8,129.8,127.8,77.4,64.3,54.7,45.3,42.2,31.3$, 28.7, 27.0, 19.3, 12.8;

IR (thin film, KBr) $v_{\max } 3427,2930,2856,1471,1427,1111 \mathrm{~cm}^{-1}$;

HRMS (EI, H) $m / z$ calc'd for $\mathrm{C}_{24} \mathrm{H}_{34} \mathrm{O}_{3} \mathrm{NaSi}[\mathrm{M}+\mathrm{Na}]^{+} 421.2175$, found 421.2161.

tert-butyl $(((4 R, 5 R)-4-m e t h o x y-5-((R)-0 x i r a n-2-y l) h e x y l) o x y) d i p h e n y l s i l a n e \quad[(-)-30]$. To a precooled $\left(0{ }^{\circ} \mathrm{C}\right)$ solution of $(+)-\mathrm{S}-8(0.535 \mathrm{~g}, 1.342 \mathrm{mmol})$ in THF $(13.5 \mathrm{~mL})$ was added $\mathrm{NaH}$ $(60 \% \mathrm{w} / \mathrm{w}$ in mineral oil, $0.161 \mathrm{~g}, 4.03 \mathrm{mmol})$. The resulting mixture was stirred at $0{ }^{\circ} \mathrm{C}$ for 10 $\min$, then MeI $(0.839 \mathrm{ml}, 13.42 \mathrm{mmol})$ was added. The reaction was then allowed to warm to $\mathrm{rt}$ and stirred for $36 \mathrm{~h}$, and finally quenched with sat. aq. $\mathrm{NH}_{4} \mathrm{Cl}(3 \mathrm{~mL})$ and $1 \mathrm{M}$ aq. $\mathrm{Na}_{2} \mathrm{~S}_{2} \mathrm{O}_{3}(3$ $\mathrm{mL})$. $\mathrm{Et}_{2} \mathrm{O}(25 \mathrm{~mL})$ was added and the layers were separated. The organic layer was dried over 
$\mathrm{Na}_{2} \mathrm{SO}_{4}$, filtered and concentrated in vacuo. Flash column chromatography (10\% EtOAc/hexanes) afforded as a clear colorless oil (551 mg, 99\%). Spectroscopic data: vide supra.

\section{Tricomponent ARC fragment union:}

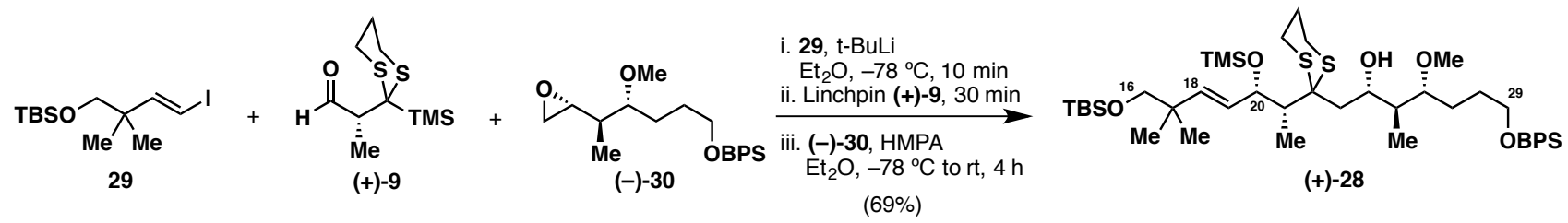

(+)-28: To a precooled $\left(-78{ }^{\circ} \mathrm{C}\right)$ solution of $29(253 \mathrm{mg}, 0.743 \mathrm{mmol})$ in $\mathrm{Et}_{2} \mathrm{O}(3 \mathrm{~mL})$ was added tert-butyllithium (1.55 M solution in pentane, $0.959 \mathrm{~mL}, 1.487 \mathrm{mmol})$ dropwise. The reaction was stirred at $-78^{\circ} \mathrm{C}$ for $10 \mathrm{~min}$. A solution of linchpin (+)-9 (180 mg, $\left.0.725 \mathrm{mmol}\right)$ in $\mathrm{Et}_{2} \mathrm{O}$ was then added dropwise via syringe $\left(1 \mathrm{~mL}+2 \times 0.5 \mathrm{~mL}\right.$ rinse). The reaction was stirred at $-78{ }^{\circ} \mathrm{C}$ for $30 \mathrm{~min}$. A solution of (-)-30 (150 mg, $0.363 \mathrm{mmol})$ in $\mathrm{Et}_{2} \mathrm{O} / \mathrm{HMPA}(1.0 \mathrm{~mL}, 10 / 1, \mathrm{v} / \mathrm{v})$, predried for 10 min over $\mathrm{CaH}_{2}$, was added dropwise via syringe, having allowed the solid to decant. The flask originally containing 30 was then carefully rinsed with $\mathrm{Et}_{2} \mathrm{O}(2 \times 0.5 \mathrm{~mL})$ to ensure complete transfer of the epoxide. The reaction was stirred at $-78{ }^{\circ} \mathrm{C}$ for $30 \mathrm{~min}$, then the cold bath was removed and the reaction was stirred for an additional $3 \mathrm{~h}$, gradually warming to rt. Sat. aq. $\mathrm{NH}_{4} \mathrm{Cl}(4 \mathrm{~mL})$ and EtOAc $(4 \mathrm{~mL})$ were added. The layers were separated and the organic layer was extracted with EtOAc $(2 \times 10 \mathrm{~mL})$. The combined organic layers were then washed with brine $(4 \mathrm{~mL})$ dried over $\mathrm{Na}_{2} \mathrm{SO}_{4}$, filtered, and concentrated in vacuo. The residue was purified by flash column chromatography (10\% EtOAc/hexanes) to furnish $\mathbf{2 8}$ as a clear colorless oil (219 mg, 69\% yield, single diastereomer, assigned by analogy with previous experiments).

$[\alpha]_{\mathbf{D}}{ }^{22}=+2.93\left(\mathrm{c} 1.0, \mathrm{CH}_{2} \mathrm{Cl}_{2}\right)$;

${ }^{1} \mathbf{H}$ NMR $\left(500 \mathrm{MHz}, \mathrm{CDCl}_{3}\right) \delta 7.72$ - $7.66(\mathrm{~m}, 4 \mathrm{H}), 7.45$ - $7.36(\mathrm{~m}, 6 \mathrm{H}), 5.66$ - $5.55(\mathrm{~m}, 1 \mathrm{H})$, $5.54-5.43(\mathrm{~m}, 1 \mathrm{H}), 5.00(\mathrm{~d}, J=6.9 \mathrm{~Hz}, 1 \mathrm{H}), 4.09(\mathrm{t}, J=8.2 \mathrm{~Hz}, 1 \mathrm{H}), 3.75-3.64(\mathrm{~m}, 2 \mathrm{H})$, 3.54 (s, 1 H), 3.41 - 3.35 (m, 1 H), 3.32 - 3.29 (m, 5 H), 2.91 - 2.80 (m, 2 H), 2.79 - 2.66 (m, 2 H), 2.18 (q, $J=6.8 \mathrm{~Hz}, 1 \mathrm{H}), 2.12-1.84(\mathrm{~m}, 5 \mathrm{H}), 1.80$ - $1.59(\mathrm{~m}, 3 \mathrm{H}), 1.47$ - $1.35(\mathrm{~m}, 1 \mathrm{H})$, $1.10(\mathrm{~d}, J=6.9 \mathrm{~Hz}, 3 \mathrm{H}), 1.06$ (s, $9 \mathrm{H}), 1.01-0.97$ (m, $6 \mathrm{H}), 0.91$ (s, $9 \mathrm{H}), 0.83$ (d, $J=6.9 \mathrm{~Hz}, 3$ H), $0.12(\mathrm{~s}, 9 \mathrm{H}), 0.04$ (s, $6 \mathrm{H})$; 
${ }^{13} \mathrm{C}$ NMR $\left(125 \mathrm{MHz}, \mathrm{CDCl}_{3}\right) \delta 137.9,135.7,134.3,131.0,129.6,127.7,81.6,73.8,72.0,70.0$, $64.3,58.1,56.8,44.8,40.9,39.7,38.1,28.6,27.0,26.1,26.0,25.6,24.8,24.2,24.0,19.4,18.4$, $10.8,8.4,1.2,-5.3,-5.4$;

IR (thin film, KBr) $v_{\max } 3429,2955,2856,1589,1471,1428,1389,1249,1105,839 \mathrm{~cm}^{-1}$; HRMS (EI, H) $m / z$ calc'd for $\mathrm{C}_{47} \mathrm{H}_{82} \mathrm{O}_{5} \mathrm{NaSi}_{3} \mathrm{~S}_{2}[\mathrm{M}+\mathrm{Na}]^{+}$897.4809, found 897.4864.

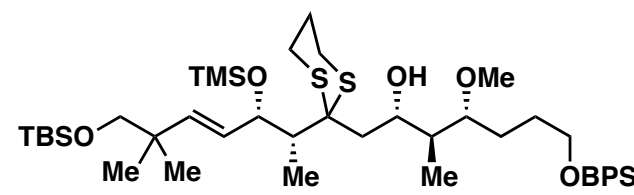

$(+)-28$

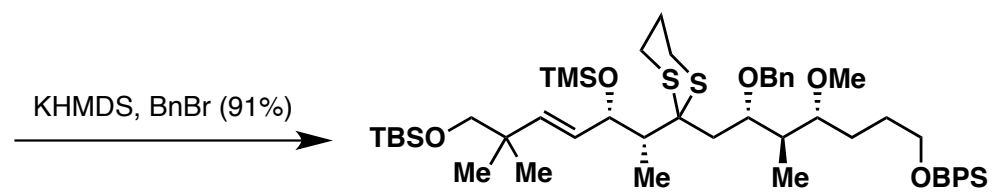

$(-)-S-9$

Benzyl ether (-)-S-9: To a precooled $\left(0^{\circ} \mathrm{C}\right)$ solution of $(+)-28(10 \mathrm{mg}, 0.011 \mathrm{mmol})$ in THF $(0.1$ $\mathrm{mL})$ were added KHMDS $(0.5 \mathrm{M}$ solution in toluene, $69 \mu \mathrm{L}, 0.034 \mathrm{mmol})$ and benzyl bromide (14 $\mu \mathrm{L}, 0.114 \mathrm{mmol}$ ). The reaction was stirred at $0{ }^{\circ} \mathrm{C}$ for $10 \mathrm{~min}$ and quenched at that temperature with sat. aq. $\mathrm{NH}_{4} \mathrm{Cl}(1 \mathrm{~mL})$. The mixture was extracted with EtOAc $(3 \times 2 \mathrm{~mL})$. The combined organic layers were washed with brine, dried over $\mathrm{Na}_{2} \mathrm{SO}_{4}$, filtered and concentrated in vacuo. The residue was purified by flash column chromatography $(1 \% \rightarrow 10 \%$ EtOAc/hexanes) to furnish (-)-S-9 as a clear colorless oil (10 mg, 91\%).

$[\alpha]_{\mathbf{D}}{ }^{22}=-14.7\left(\mathrm{c} 0.5, \mathrm{CH}_{2} \mathrm{Cl}_{2}\right)$;

${ }^{1}$ H NMR $\left(500 \mathrm{MHz}, \mathrm{CDCl}_{3}\right) \delta 7.71-7.67$ (m, $\left.4 \mathrm{H}\right), 7.45-7.36(\mathrm{~m}, 8 \mathrm{H}), 7.30(\mathrm{t}, J=7.4 \mathrm{~Hz}, 2$ H), 7.26 - $7.19(\mathrm{~m}, 1 \mathrm{H}), 5.58(\mathrm{~d}, J=15.9 \mathrm{~Hz}, 1 \mathrm{H}), 5.47(\mathrm{dd}, J=6.7,14.9 \mathrm{~Hz}, 1 \mathrm{H}), 5.11(\mathrm{~d}, \mathrm{~J}=$ $6.7 \mathrm{~Hz}, 1 \mathrm{H}), 4.78$ (d, J = 11.5 Hz, $1 \mathrm{H}), 4.50$ (d, J = 11.7 Hz, $1 \mathrm{H}), 4.19$ (dd, J=2.3, 8.2 Hz, 1 H), $3.70(\mathrm{t}, J=6.0 \mathrm{~Hz}, 2 \mathrm{H}), 3.37$ - 3.28 (m, $5 \mathrm{H}), 3.12$ - 3.04 (m, $1 \mathrm{H}), 2.96$ - 2.87 (m, $1 \mathrm{H}), 2.82$ - $2.73(\mathrm{~m}, 1 \mathrm{H}), 2.67$ - 2.52 (m, 2 H), 2.31 - 2.21 (m, 2 H), 2.13 - 2.04 (m, 1 H), 1.95 (br. s., 1 H), $1.91-1.80(\mathrm{~m}, 2 \mathrm{H}), 1.76(\mathrm{~d}, J=16.1 \mathrm{~Hz}, 1 \mathrm{H}), 1.70-1.47\left(\mathrm{~m}, 3 \mathrm{H}+\mathrm{H}_{2} \mathrm{O}\right), 1.18-1.11(\mathrm{~m}, 3 \mathrm{H})$, 1.07 (s, 9 H), 0.97 (br. s, 6 H), 0.91 (s, 9 H), 0.84 (d, J=6.7 Hz, 3 H), 0.10 (s, 9 H), 0.04 (s, 6 H); ${ }^{13}$ C NMR $\left(125 \mathrm{MHz}, \mathrm{CDCl}_{3}\right) \delta 140.3,137.4,135.7,134.2,131.5,129.6,128.0,127.7,127.4$, $126.8,81.5,76.8,73.7,72.1,69.9,64.4,59.1,56.6,43.5,38.2,38.0,36.1,29.8,27.0,26.8,26.6$, $26.1,25.8,25.6,25.0,24.3,24.1,19.4,18.5,10.5,8.4,1.1,-5.3$;

IR (thin film, KBr) $v_{\max } 3434,2928,2845,1653,1472,1249,1105,839,701 \mathrm{~cm}^{-1}$; HRMS (EI, H) $m / z$ calc'd for $\mathrm{C}_{54} \mathrm{H}_{88} \mathrm{O}_{5} \mathrm{NaSi}_{3} \mathrm{~S}_{2}[\mathrm{M}+\mathrm{Na}]^{+}$987.5279, found 987.5242. 
(-)-S-9': Minor position isomer (analytical sample)

${ }^{1}$ H NMR $\left(500 \mathrm{MHz}, \mathrm{CDCl}_{3}\right) \delta 7.71-7.66(\mathrm{~m}, 4 \mathrm{H}), 7.46$

- $7.35(\mathrm{~m}, 8 \mathrm{H}), 7.30$ (t, $J=7.5 \mathrm{~Hz}, 2 \mathrm{H}), 7.25-7.20$ (m,

$1 \mathrm{H}), 5.70(\mathrm{~d}, J=15.9 \mathrm{~Hz}, 1 \mathrm{H}), 5.47(\mathrm{dd}, J=7.1,15.9$

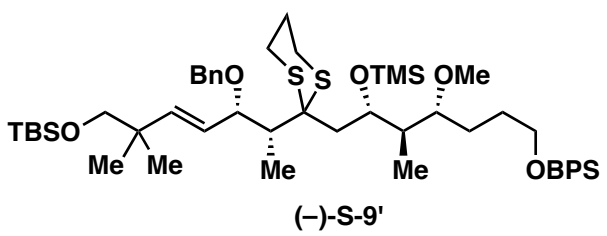

Hz, $1 \mathrm{H}), 4.63(\mathrm{~d}, J=6.9 \mathrm{~Hz}, 1 \mathrm{H}), 4.55-4.42(\mathrm{~m}, 2 \mathrm{H}), 4.38$ (d, $J=11.5 \mathrm{~Hz}, 1 \mathrm{H}), 3.68(\mathrm{t}, J=$ $6.2 \mathrm{~Hz}, 2 \mathrm{H}), 3.37$ - 3.31 (m, 2 H), 3.29 (s, $3 \mathrm{H}), 3.03$ (td, $J=4.4,8.3 \mathrm{~Hz}, 1 \mathrm{H}), 2.92$ - 2.60 (m, 4 H), 2.39 (q, $J=7.1 \mathrm{~Hz}, 1 \mathrm{H}), 2.11(\mathrm{dd}, \mathrm{J}=8.1,16.4 \mathrm{~Hz}, 1 \mathrm{H}), 2.04-1.69(\mathrm{~m}, 5 \mathrm{H}), 1.61(\mathrm{td}, J=$ 7.0, $14.4 \mathrm{~Hz}, 2 \mathrm{H}), 1.51-1.41(\mathrm{~m}, 1 \mathrm{H}), 1.20$ (d, $J=7.1 \mathrm{~Hz}, 3 \mathrm{H}), 1.07$ (s, $9 \mathrm{H}), 1.03-0.98$ (m, 6 H), 0.91 (s, 9 H), 0.78 - 0.72 (m, $3 \mathrm{H}), 0.17$ (s, $9 \mathrm{H}), 0.04$ (s, $6 \mathrm{H}$ );

${ }^{13}$ C NMR $\left(125 \mathrm{MHz}, \mathrm{CDCl}_{3}\right) \delta 139.9,139.4,135.7,134.2,129.6,128.2,127.9,127.7,127.6$, 127.1, 81.9, 80.1, 72.1, 70.4, 70.3, 64.4, 58.8, 56.4, 43.7, 43.2, 38.4, 29.9, 27.1, 27.0, 26.6, 26.2, 26.1, 26.0, 25.2, 24.4, 19.4, 18.4, 10.3, 9.1, 1.3, -5.3, -5.3;

IR (thin film, KBr) $v_{\max } 2954,2857,1471,1428,1388,1248,1105,838,775,701 \mathrm{~cm}^{-1}$;

HRMS (EI, H) $m / z$ calc'd for $\mathrm{C}_{54} \mathrm{H}_{89} \mathrm{O}_{5} \mathrm{Si}_{3} \mathrm{~S}_{2}[\mathrm{M}+\mathrm{H}]^{+}$965.5459, found 965.5470 .

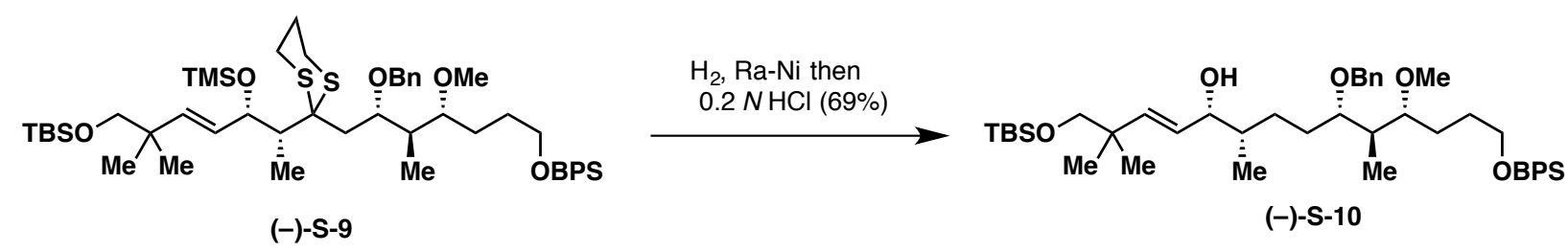

Alcohol (-)-S-10: THF was degassed by sparging with $\mathrm{N}_{2}$ during 15 min prior to the reaction. Raney nickel (W. R. Grace $2800,0.5 \mathrm{~mL}$ of a $89 \%$ slurry in $\mathrm{H}_{2} \mathrm{O}$, excess) was added via syringe to a $50-\mathrm{mL}$ round-bottom flask fitted with a septum and stir bar under $\mathrm{N}_{2}$ atmosphere. Water was removed via syringe, and the nickel was rinsed with water $(4 \times 4 \mathrm{~mL})$, then with degassed THF $(4 \times 4 \mathrm{~mL})$, stirring vigorously between each rinse. Degassed THF $(2 \mathrm{~mL})$ and a solution of (-)S-9 $(39 \mathrm{mg}, 0.040 \mathrm{mmol})$ in THF $(2 \mathrm{~mL})$ were then added. The flask was then evacuated and backfilled with $\mathrm{H}_{2}$ (x 5), fitted with a reflux condenser, and warmed to $75^{\circ} \mathrm{C}$. The suspension was stirred vigorously at $75^{\circ} \mathrm{C}$ under $\mathrm{H}_{2}$ atmosphere for $2 \mathrm{~h}$. The reaction was allowed to cool to rt and the headspace of the flask was flushed with $\mathrm{N}_{2}$. The supernatant was filtered through Celite, eluting with EtOAc, and rinsing the metal residue with EtOAc $(4 \times 4 \mathrm{~mL})$ taking special care never to leave any metal residue dry and exposed to air - risk of fire. The filtrate was concentrated in vacuo and the residue taken up in THF $(4 \mathrm{~mL})$. Aq. $\mathrm{HCl}(0.2 \mathrm{~N}, 4 \mathrm{~mL})$ was then 
added, and the biphasic mixture was stirred vigorously for $5 \mathrm{~min}$. Sat. aq. $\mathrm{NaHCO}_{3}(2 \mathrm{~mL})$ was then added, the layers partitioned, and the aqueous layer extracted with EtOAc $(3 \times 3 \mathrm{~mL})$. The organic layers were combined, washed with brine $(2 \mathrm{~mL})$, dried over $\mathrm{Na}_{2} \mathrm{SO}_{4}$, filtered and concentrated in vacuo. The residue was purified by flash column chromatography (5\% EtOAc/hexane) to furnish $\mathbf{S - 1 0}$ as a clear colorless oil (22 $\mathrm{mg}, 69 \%$ ).

$[\alpha]_{\mathbf{D}}^{22}=-12.1\left(\mathrm{c} 0.33, \mathrm{CH}_{2} \mathrm{Cl}_{2}\right)$;

${ }^{1}$ H NMR $\left(500 \mathrm{MHz}, \mathrm{CDCl}_{3}\right) \delta 7.68(\mathrm{~d}, \mathrm{~J}=7.1 \mathrm{~Hz}, 4 \mathrm{H}), 7.46-7.30(\mathrm{~m}, 11 \mathrm{H}), 5.65(\mathrm{~d}, J=15.9$

Hz, $1 \mathrm{H}), 5.44$ (dd, $J=6.8,15.8 \mathrm{~Hz}, 1 \mathrm{H}), 4.46$ (s, $2 \mathrm{H}), 3.98$ - 3.91 (m, $1 \mathrm{H}), 3.72$ - 3.63 (m, 2

H), $3.48(\mathrm{~d}, J=5.2 \mathrm{~Hz}, 1 \mathrm{H}), 3.32$ - 3.20 (m, $6 \mathrm{H}), 2.16$ - 2.08 (m, $1 \mathrm{H}), 1.80$ - 1.67 (m, $1 \mathrm{H})$, 1.65 - 1.47 (m, $8 \mathrm{H}), 1.46$ - 1.35 (m, $2 \mathrm{H}), 1.32$ - 1.22 (m, $2 \mathrm{H}), 1.06$ (s, $9 \mathrm{H}), 0.98$ (d, J = 5.5 Hz, $6 \mathrm{H}), 0.90(\mathrm{~s}, 9 \mathrm{H}), 0.81(\mathrm{~d}, J=7.1 \mathrm{~Hz}, 3 \mathrm{H}), 0.02(\mathrm{~s}, 6 \mathrm{H})$;

${ }^{13}$ C NMR $\left(125 \mathrm{MHz}, \mathrm{CDCl}_{3}\right) \delta 139.9,139.2,135.7,134.2,129.7,128.4,127.9,127.7,127.5$, $81.8,80.0,77.2,72.0,71.4,64.3,56.8,39.0,38.3,37.5,29.8,28.3,28.0,27.5,27.0,26.1,26.0$, 24.2, 24.1, 19.4, 18.4, 14.7, 10.3, -5.3;

IR (thin film, KBr) $v_{\max } 3441,2926,2855,1462,1384,1094,836,701 \mathrm{~cm}^{-1}$;

HRMS (EI, H) $m / z$ calc'd for $\mathrm{C}_{48} \mathrm{H}_{76} \mathrm{O}_{5} \mathrm{NaSi}_{2}[\mathrm{M}+\mathrm{Na}]^{+} 811.5129$, found 811.5109.

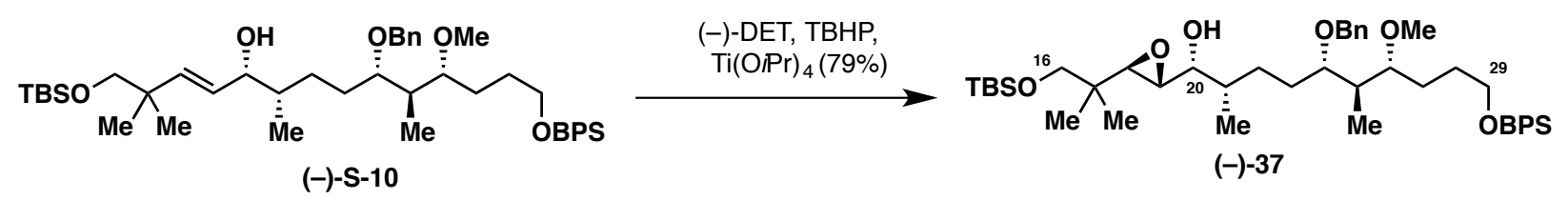

Epoxide (-)-37: To a precooled $\left(-20^{\circ} \mathrm{C}\right)$, stirred suspension of flame-dried molecular sieve (4 $\AA$, powder, $60 \mathrm{mg})$ in $\mathrm{CH}_{2} \mathrm{Cl}_{2}(0.2 \mathrm{~mL})$ were added a solution of (-)-diethyl-D-tartrate in $\mathrm{CH}_{2} \mathrm{Cl}_{2}$ $(90 \mu \mathrm{L}, 0.2 \mathrm{M}, 0.018 \mathrm{mmol})$ and a solution of titanium(IV) isopropoxide in $\mathrm{CH}_{2} \mathrm{Cl}_{2}(75 \mu \mathrm{L}, 0.2$ $\mathrm{M}, 0.015 \mathrm{mmol})$. The suspension was stirred for $30 \mathrm{~min}$ at $-20^{\circ} \mathrm{C}$. Was then added tert-butyl hydroperoxide $(5.5 \mathrm{M}$ solution in nonane over molecular sieve, $10 \mu \mathrm{L}, 0.055 \mathrm{mmol})$, and the mixture was stirred for an additional $30 \mathrm{~min}$ at $-20{ }^{\circ} \mathrm{C}$. A solution of $(-)-\mathbf{S}-\mathbf{1 0}(6 \mathrm{mg}, 7.60 \mu \mathrm{mol})$ in $\mathrm{CH}_{2} \mathrm{Cl}_{2}(0.1 \mathrm{~mL}+2 \times 0.1 \mathrm{~mL}$ rinse $)$ was finally added dropwise via syringe. The reaction was warmed to $0{ }^{\circ} \mathrm{C}$ and stirred overnight. $\mathrm{NaOH}(1 \mathrm{~mL}, 0.5 \mathrm{M}$ in brine) was then added. The cold bath was removed and the reaction stirred for $10 \mathrm{~min}$ at $\mathrm{rt}$. The mixture was extracted with 
$\mathrm{CH}_{2} \mathrm{Cl}_{2}(3 \times 3 \mathrm{~mL})$, dried over $\mathrm{Na}_{2} \mathrm{SO}_{4}$, filtered and concentrated. The residue was purified by flash column chromatography (10\% EtOAc/hexanes) to afford $\mathbf{3 7}$ as a clear colorless oil ( $4.8 \mathrm{mg}$, $79 \%)$. Stereochemical configuration assigned based on literature precedent. ${ }^{9}$

$[\alpha]_{\mathbf{D}}{ }^{22}=-7.63\left(\mathrm{c} 0.34, \mathrm{CH}_{2} \mathrm{Cl}_{2}\right)$;

${ }^{1} \mathbf{H}$ NMR $\left(500 \mathrm{MHz}, \mathrm{CDCl}_{3}\right) \delta 7.70$ - $7.65(\mathrm{~m}, 4 \mathrm{H}), 7.45$ - $7.35(\mathrm{~m}, 6 \mathrm{H}), 7.35$ - $7.29(\mathrm{~m}, 4 \mathrm{H})$, 7.26 - 7.23 (m, 1 H), 4.46 (s, 2 H), 3.75 - 3.62 (m, 3 H), 3.52 - 3.44 (m, 1 H), 3.43 - 3.32 (m, 2 H), 3.29 - 3.26 (m, 3 H), $3.25-3.20(\mathrm{~m}, 1 \mathrm{H}), 2.99$ - 2.94 (m, $2 \mathrm{H}), 2.13$ (q, J = 6.8 Hz, $1 \mathrm{H}$ ), $1.77-1.17(\mathrm{~m}, 10 \mathrm{H}), 1.06$ (s, $9 \mathrm{H}), 0.98(\mathrm{~d}, \mathrm{~J}=6.6 \mathrm{~Hz}, 3 \mathrm{H}), 0.91(\mathrm{~s}, 9 \mathrm{H}), 0.88(\mathrm{~s}, 3 \mathrm{H}), 0.86$ (s, $3 \mathrm{H}), 0.81(\mathrm{~d}, \mathrm{~J}=7.1 \mathrm{~Hz}, 3 \mathrm{H}), 0.06-0.04(\mathrm{~m}, 6 \mathrm{H})$;

${ }^{13} \mathbf{C}$ NMR $\left(125 \mathrm{MHz}, \mathrm{CDCl}_{3}\right) \delta$

139.2, 135.7, 134.3, 129.7, 128.4, 127.9, 127.7, 127.5, 81.8, 80.0, 72.2, 71.4, 69.6, 64.4, 60.1, $56.9,56.8,37.5,36.4,35.9,29.9,28.8,28.4,27.4,27.1,26.2,26.1,20.9,20.5,19.4,18.5,14.3$, $14.3,10.3,-5.4,-5.4$

IR (thin film, KBr) $v_{\max } 3416,2925,2853,1462,1383,1095,837,701 \mathrm{~cm}^{-1}$;

HRMS (EI, H) $m / z$ calc'd for $\mathrm{C}_{48} \mathrm{H}_{76} \mathrm{O}_{6} \mathrm{NaSi}_{2}[\mathrm{M}+\mathrm{Na}]^{+} 827.5078$, found 827.5098. 


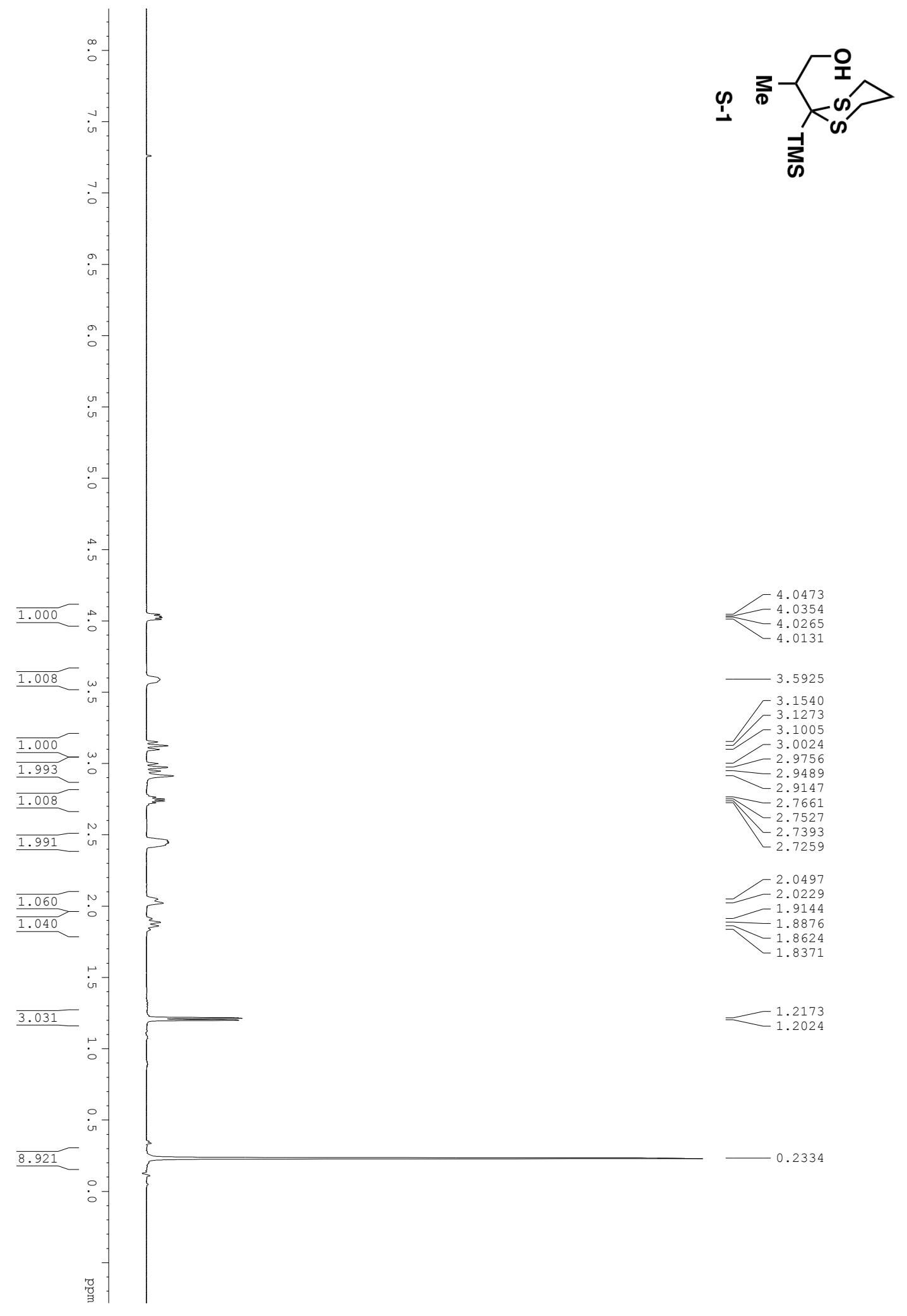




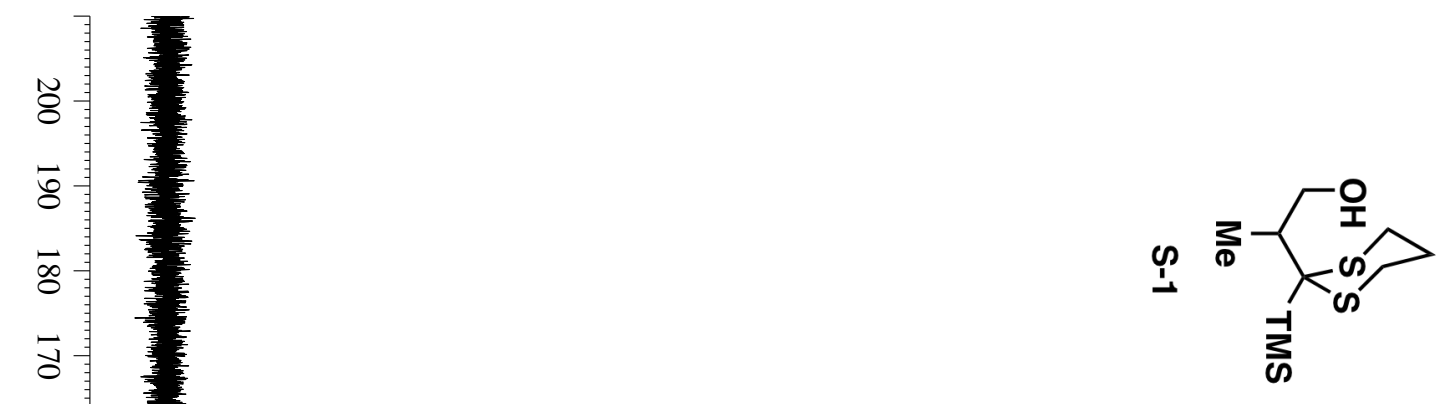

$\bar{a}$

प्a

E

产

స్

ప

$\overline{8}$

8

$\infty$

d

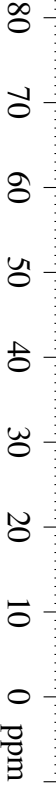

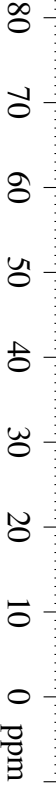

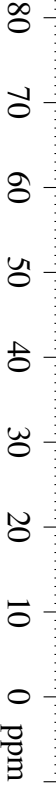

w

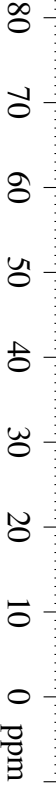

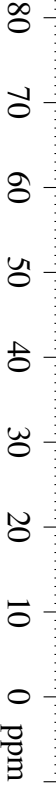

$\circ$

윸

章

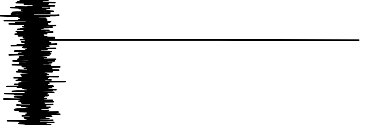

77.410

77.155

76.900

$-67.417$

42.529

42.069

24.477

24.173

23.729

17.050

$-0.283$ 


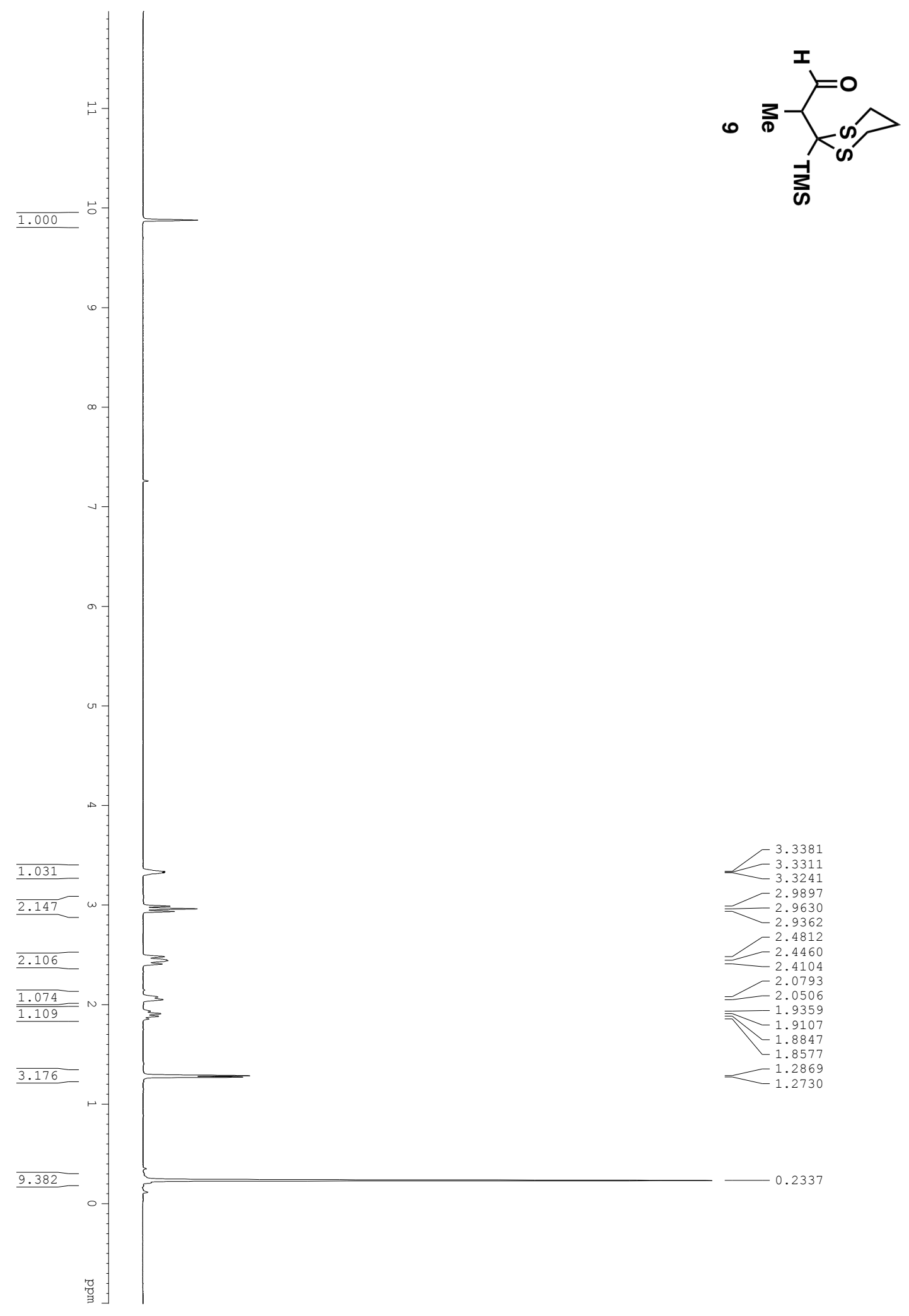



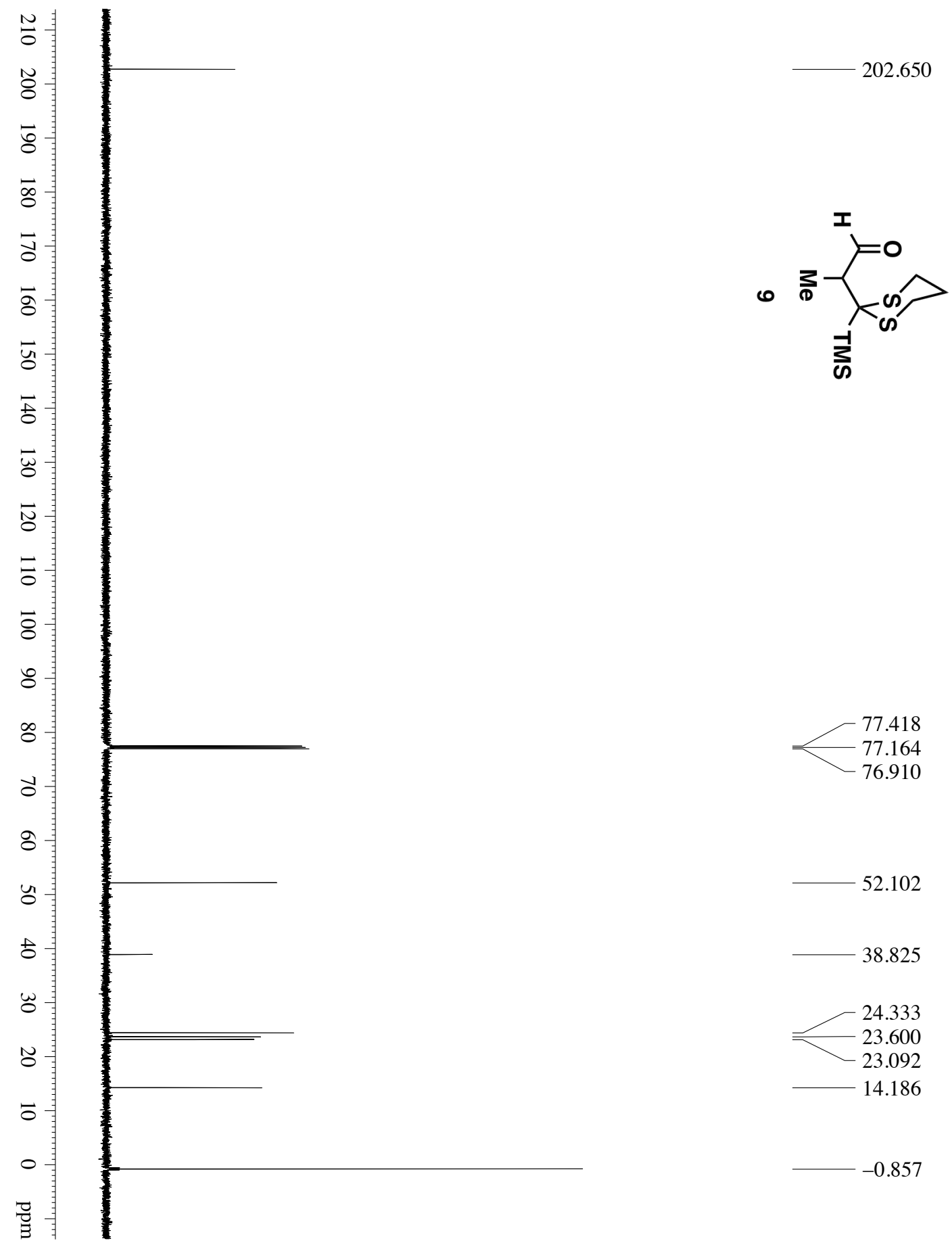

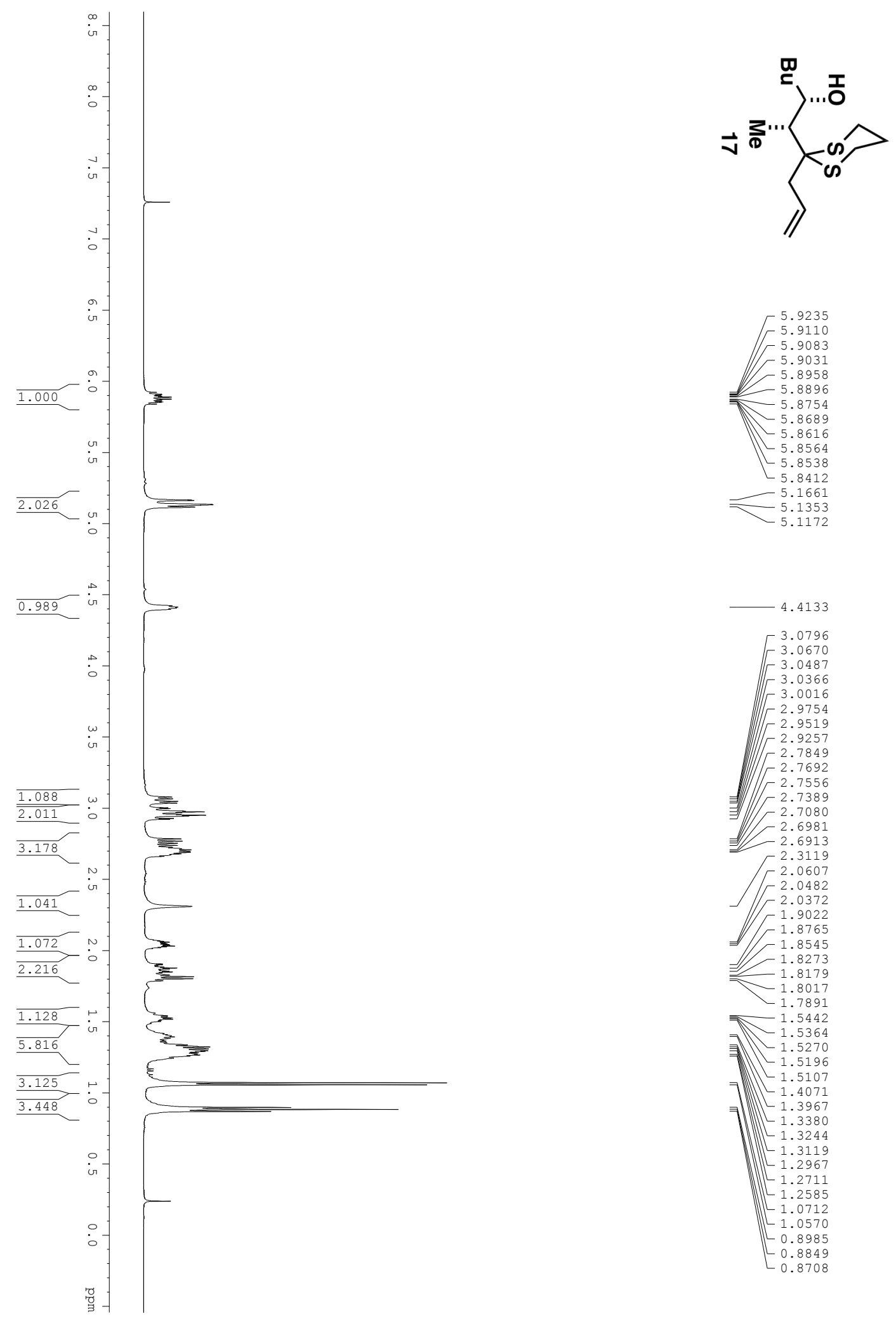

-3.0796 3.0670 3.0487
3.0366 3.0366
3.0016 F 3.0016 UIF 2.9754 广2.9257 -2.7849 $\Gamma^{2.7692}$ 2.7596
-2.7389
-2.7080 当/ $/ F^{2.7389} 2.7080$ $\begin{array}{r}2.6981 \\ -2.6913 \\ \hline\end{array}$ $\Gamma^{2} .3119$ $-2.0482$ $\sqrt{2.0372}$ $F^{1.9022}$ $-1.8545$ 1.8273
-1.8179 $\begin{array}{r}1.8017 \\ \times 1.7891 \\ \hline\end{array}$ 1.5442
-1.5364
-1.5270 灵 -1.5270 WL 1.5107 $L_{1.3967}$ $7-1.3380$ $\left[\begin{array}{l}1.324 \\ -1.319\end{array}\right.$ $-1.3119$ $[-1.2967$ $-1.2711$ $-1.2585$ $-1.0712$ $-1.0570$ $-0.8985$ $-0.8849$ 

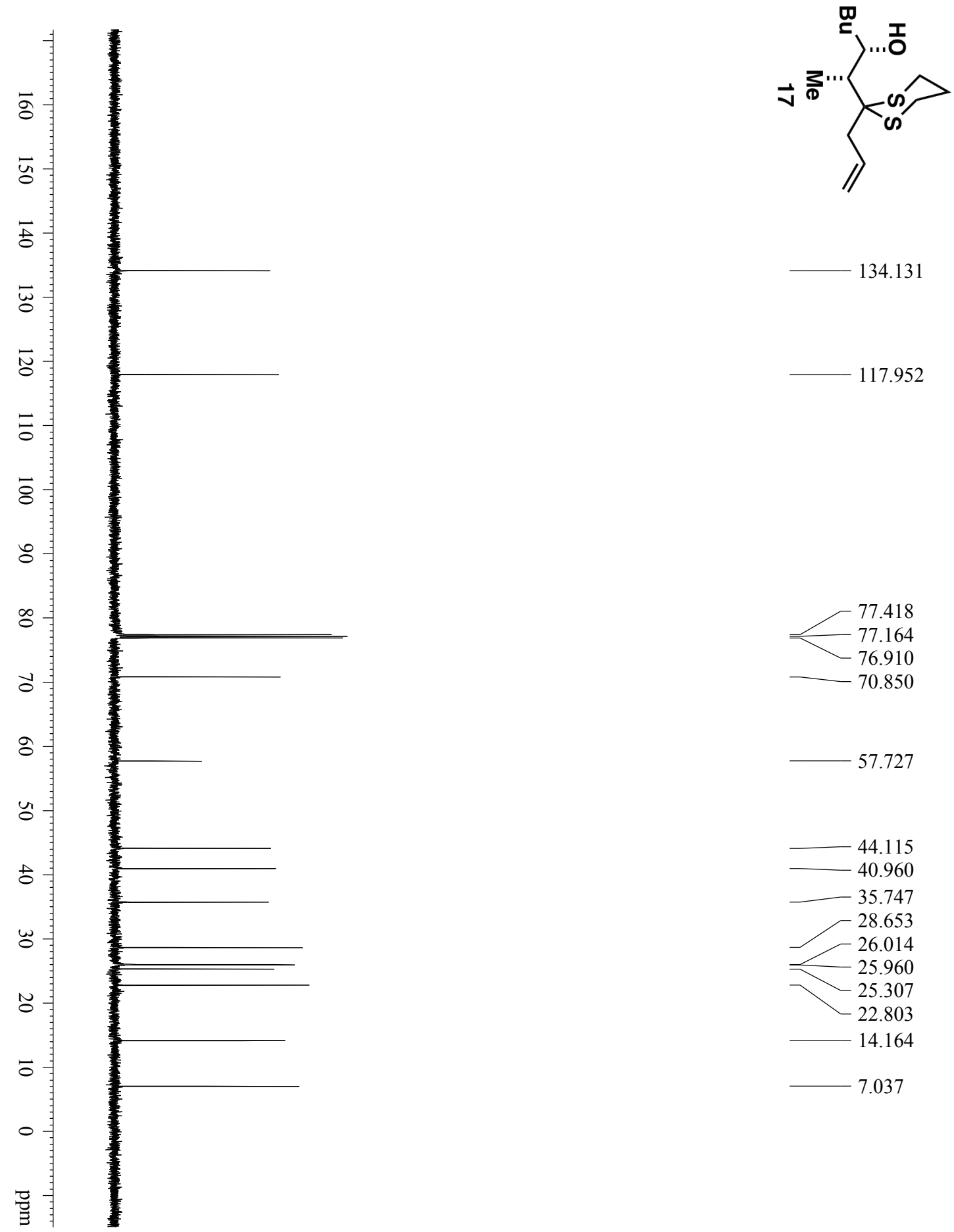

134.131

117.952

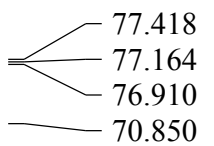

57.727

44.115

40.960

35.747

28.653

26.014

25.960

$-25.307$

22.803

14.164

7.037 

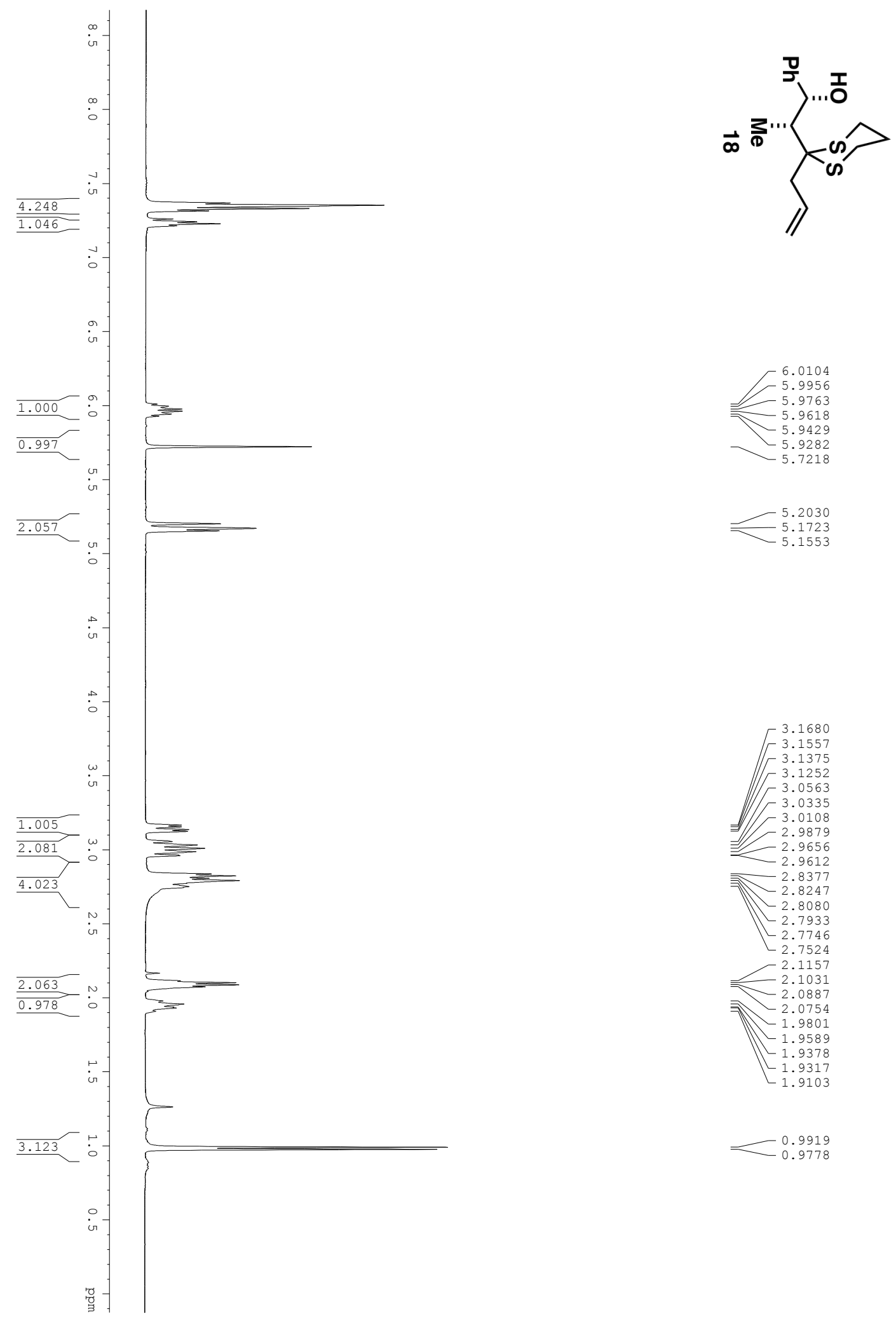

S-33 

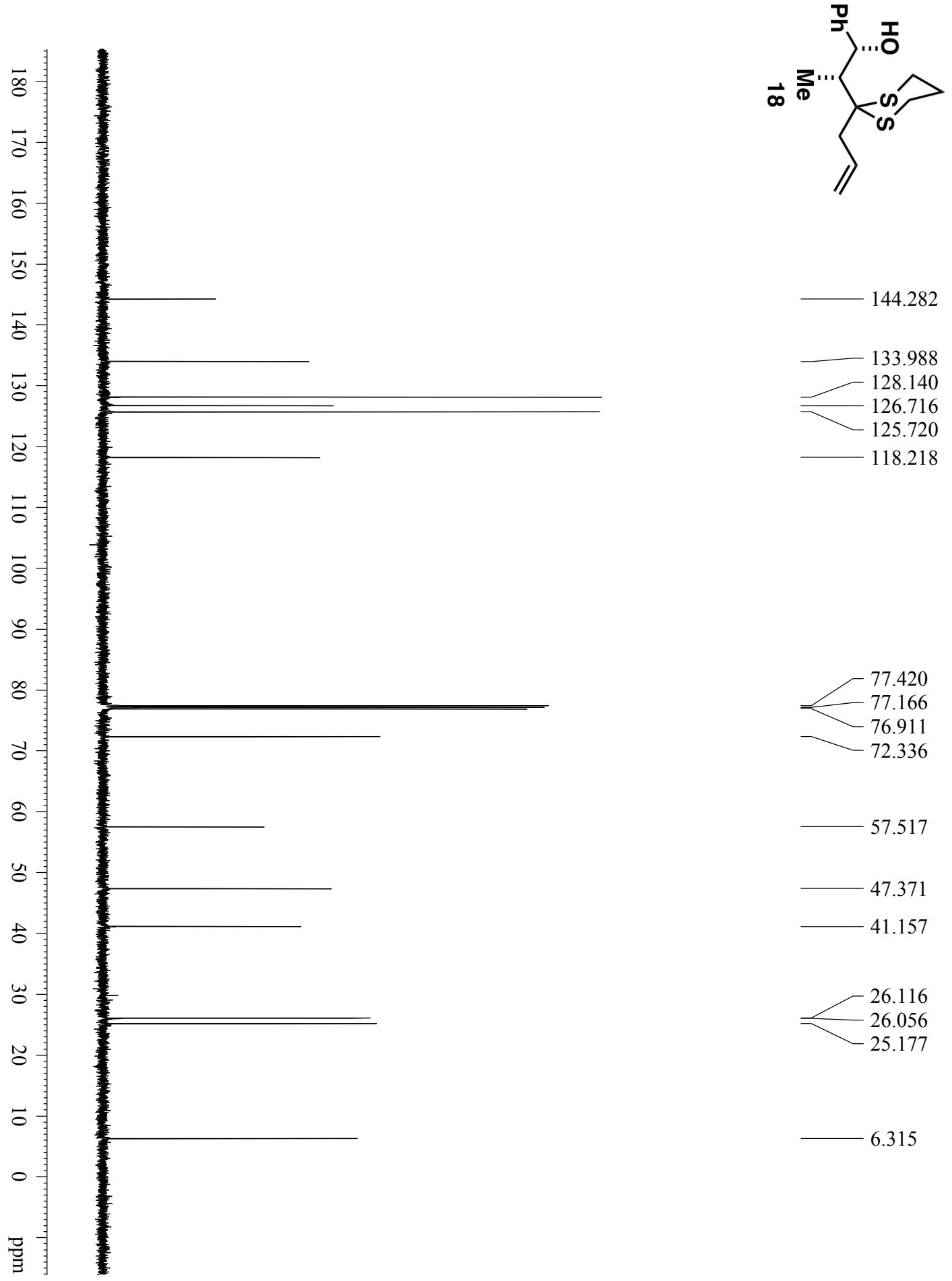

- 57.517

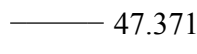

41.157

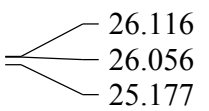

$-6.315$ 


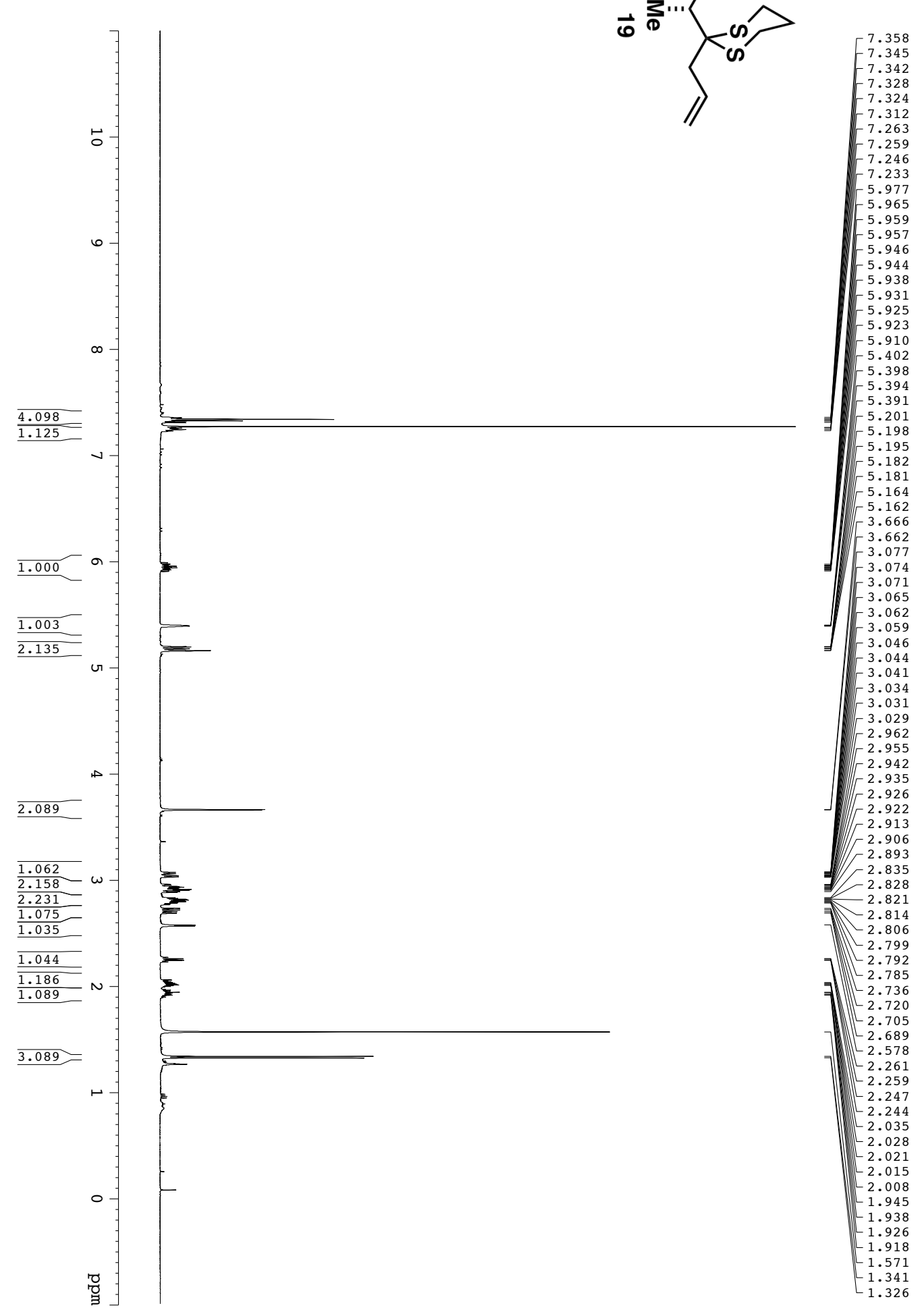



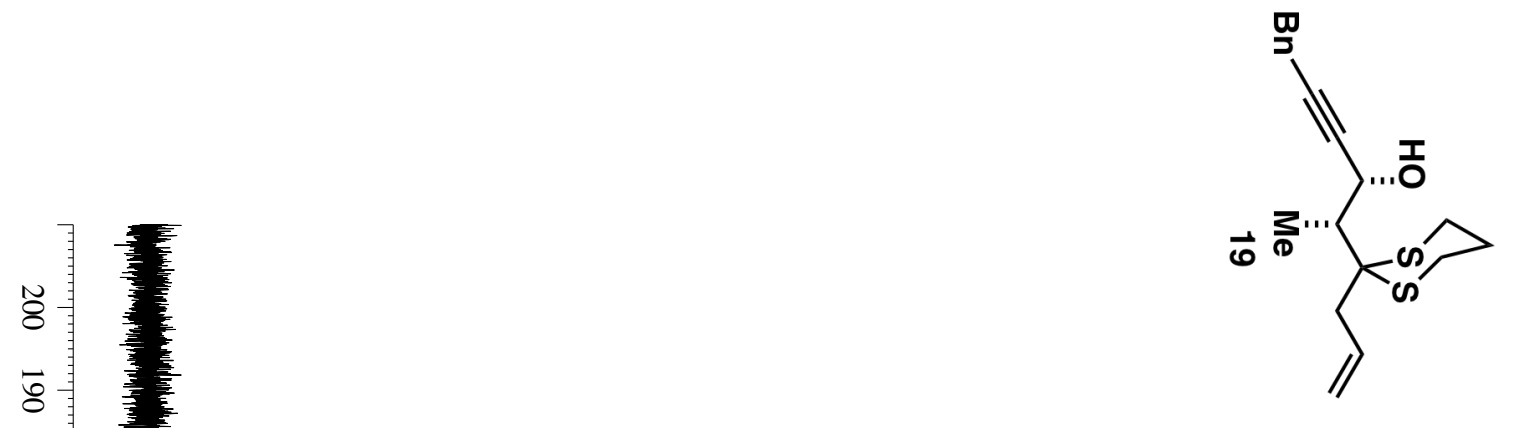

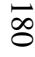

깅

8

$\bar{v}$

$\bar{\omega}$

్ㅡㅇ

๘

8

๖

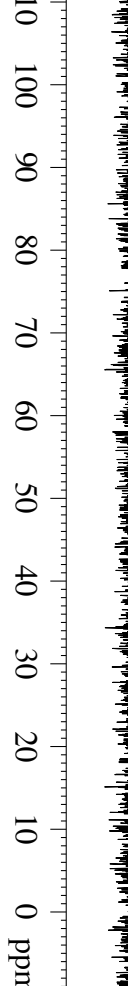

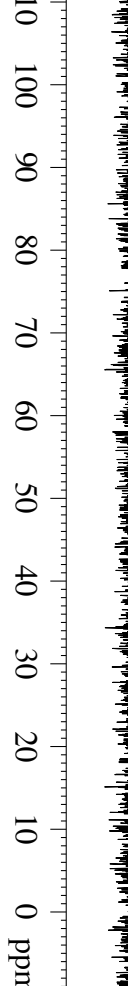

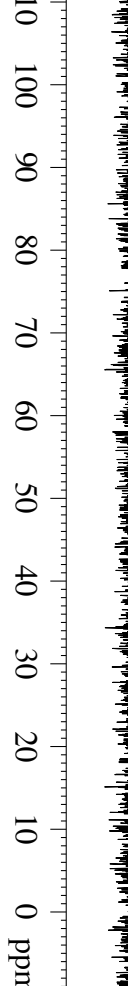

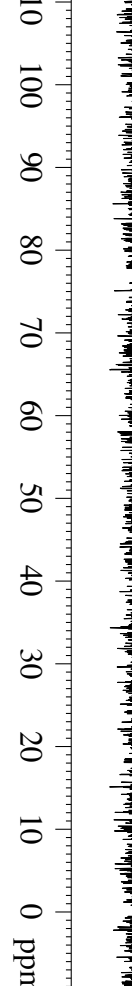

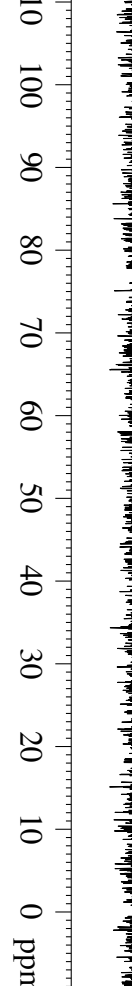

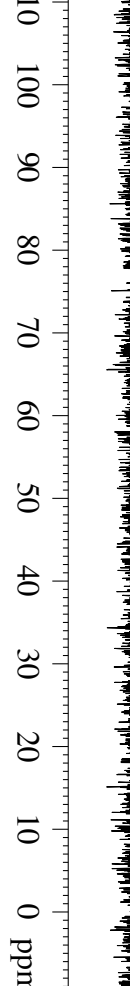

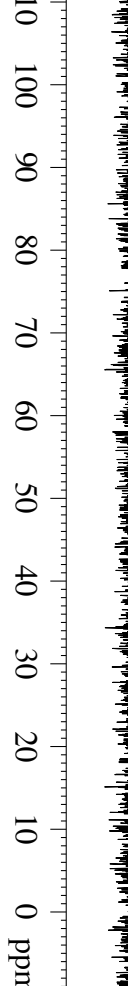

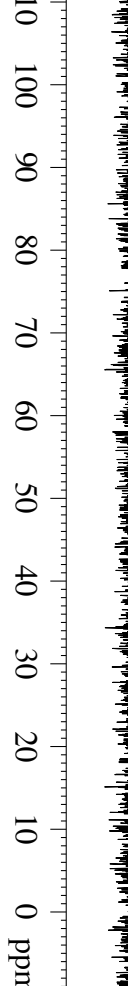

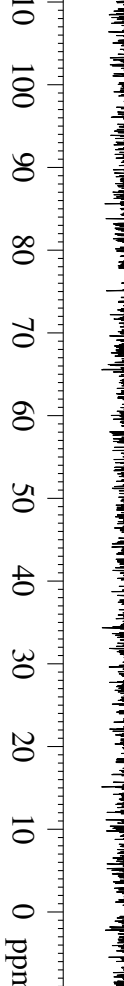

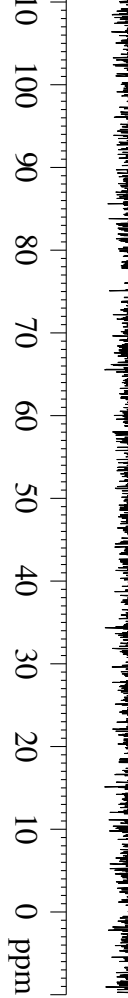

$\vec{t}$

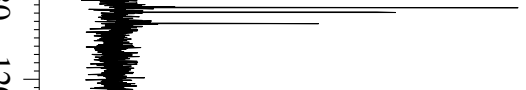

$-136.874$

134.534

128.687

128.590

126.684

$\exists+$ 

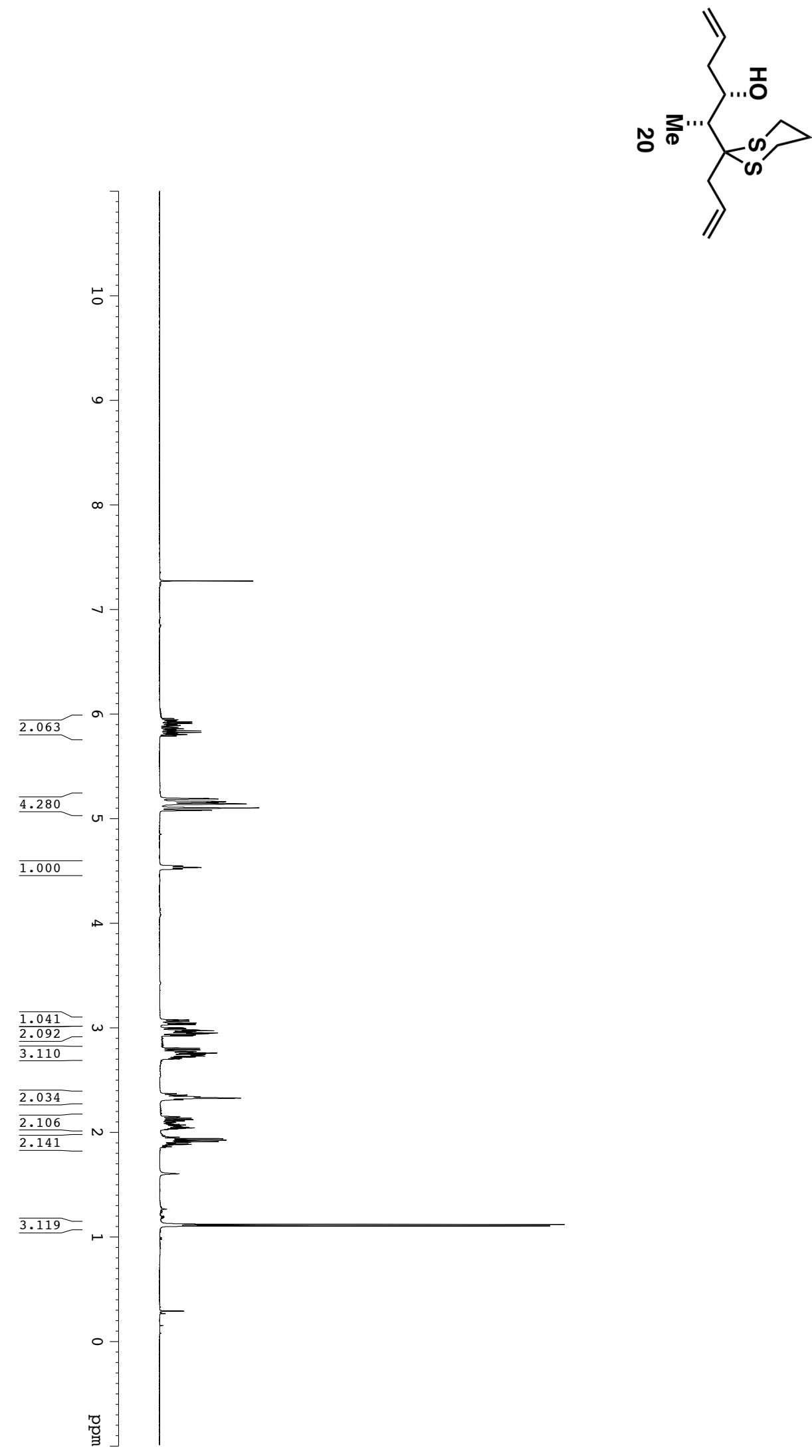

$\left[\begin{array}{r}5.94 \\ -5.92 \\ -5.911\end{array}\right.$

-5.911
-5.892

-5.892
-5.890

-5.890
-5.877

$-5.860$

$-5.840$

$-5.819$

$-5.805$

$-5.193$

$-5.190$

5.165

5.162

5.159

5.155

5.144

5.141

5.103

5.100

$-4.534$

3.062

$-3.047$

-3.047
-3.042

3.042

3.034
3.032

3.032 3.029
-2.999 - 2.993 -2.979
-2.971 $-2.971$ -2.959
-2.959 $-2.945$ 2.930
-2.924 2.924
-2.804 $-2.804$ $-2.789$ 2.77 $-2.771$ $-2.750$ 2.741 2.738
-2.736 $-2.730$ 2.721
-2.714 $-2.714$ 2.709
2.356 2.356 $\left[\begin{array}{r}2.353 \\ 2.341\end{array}\right.$ 2.315
-2.312 2.312
-2.150 $\digamma^{2} .150$ $-2.137$ -2.137
-2.135 2.135
-2.123 2.123
-2.109 $-2.076$ 2.070 2.063 2.054 2.052 2.048 2.042
2.036 1.954 1.940 1.933 $-1.926$ [ 1.919 $-1.912$ $-1.905$ 1.89 $\left[\begin{array}{l}1.884 \\ 1.119\end{array}\right.$ $\left[\begin{array}{l}1.119 \\ 1.105\end{array}\right.$ 

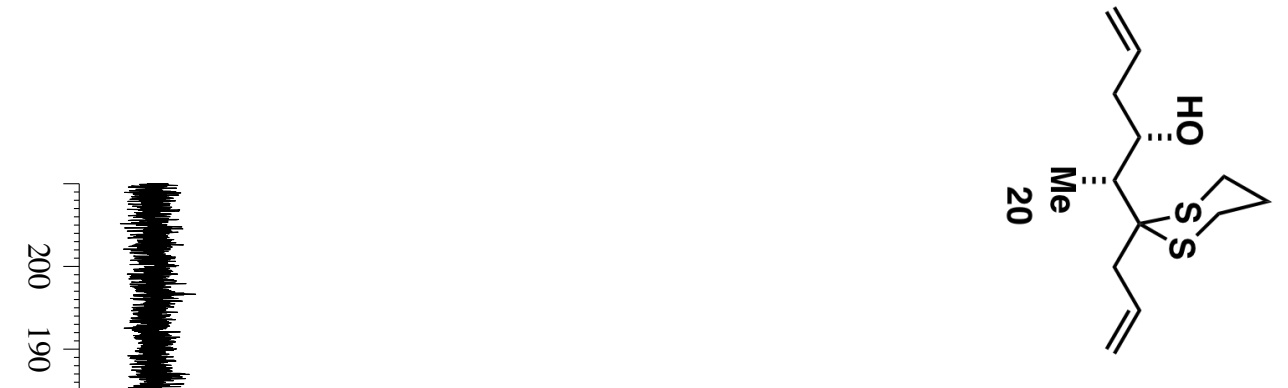

$\ddot{\circ}$

깅

$\overline{8}$

강

\&

फ़

స

$\bar{\sigma}$

$\overline{8}$

8

$\infty$

ठ઼

8

पू

b

w

ㅇ

o

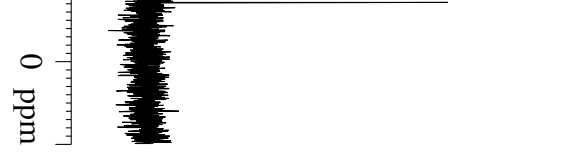

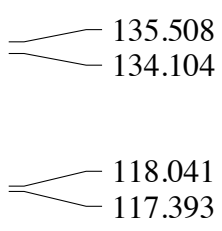

77.416

77.162

76.907

70.210

57.700

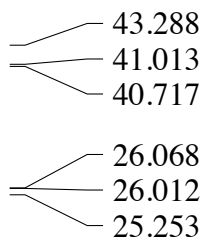

7.081 


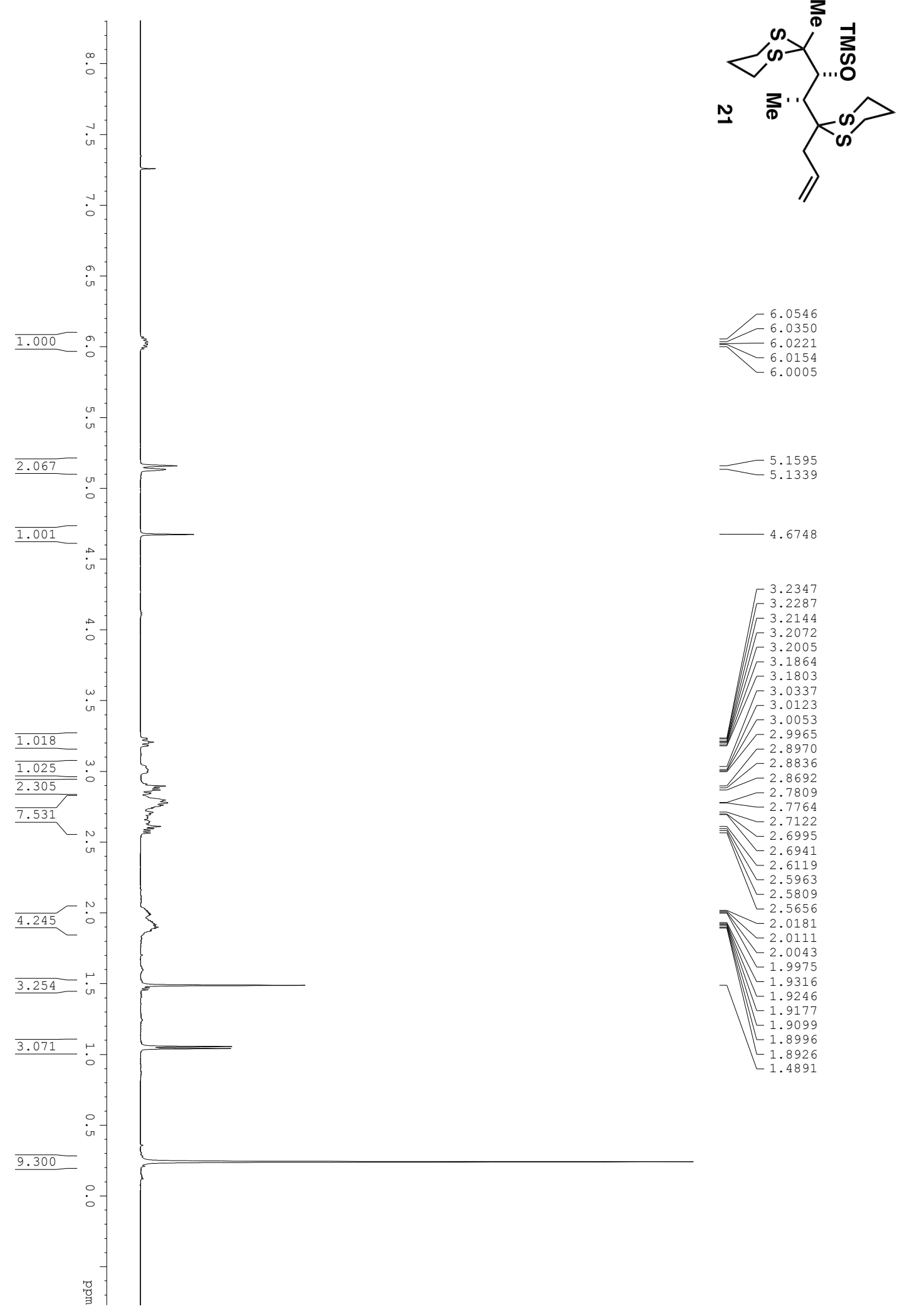



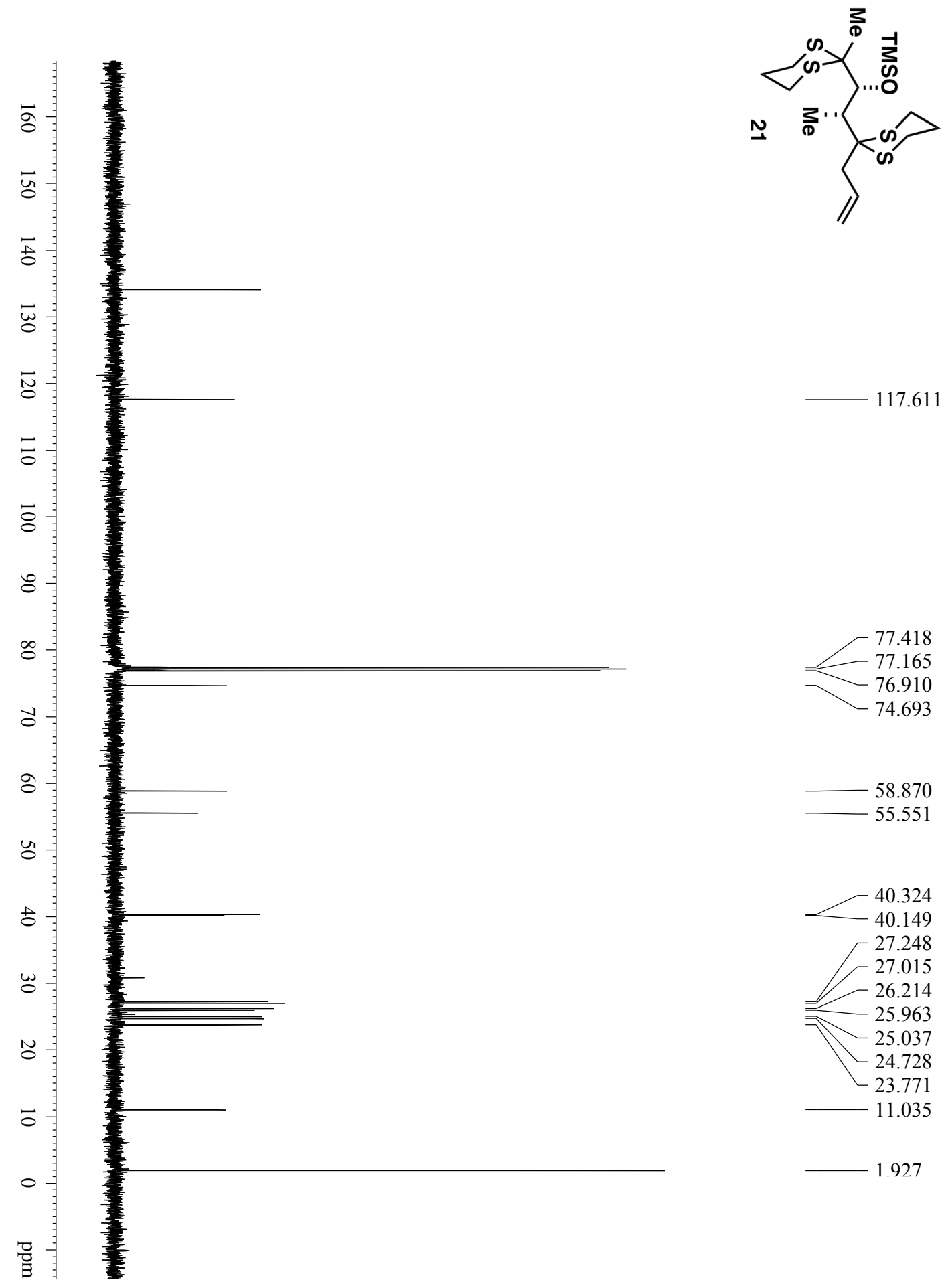

- 117.611
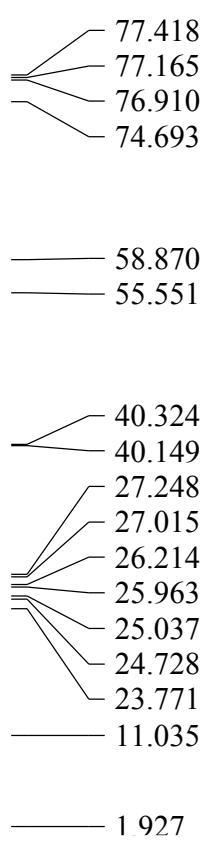

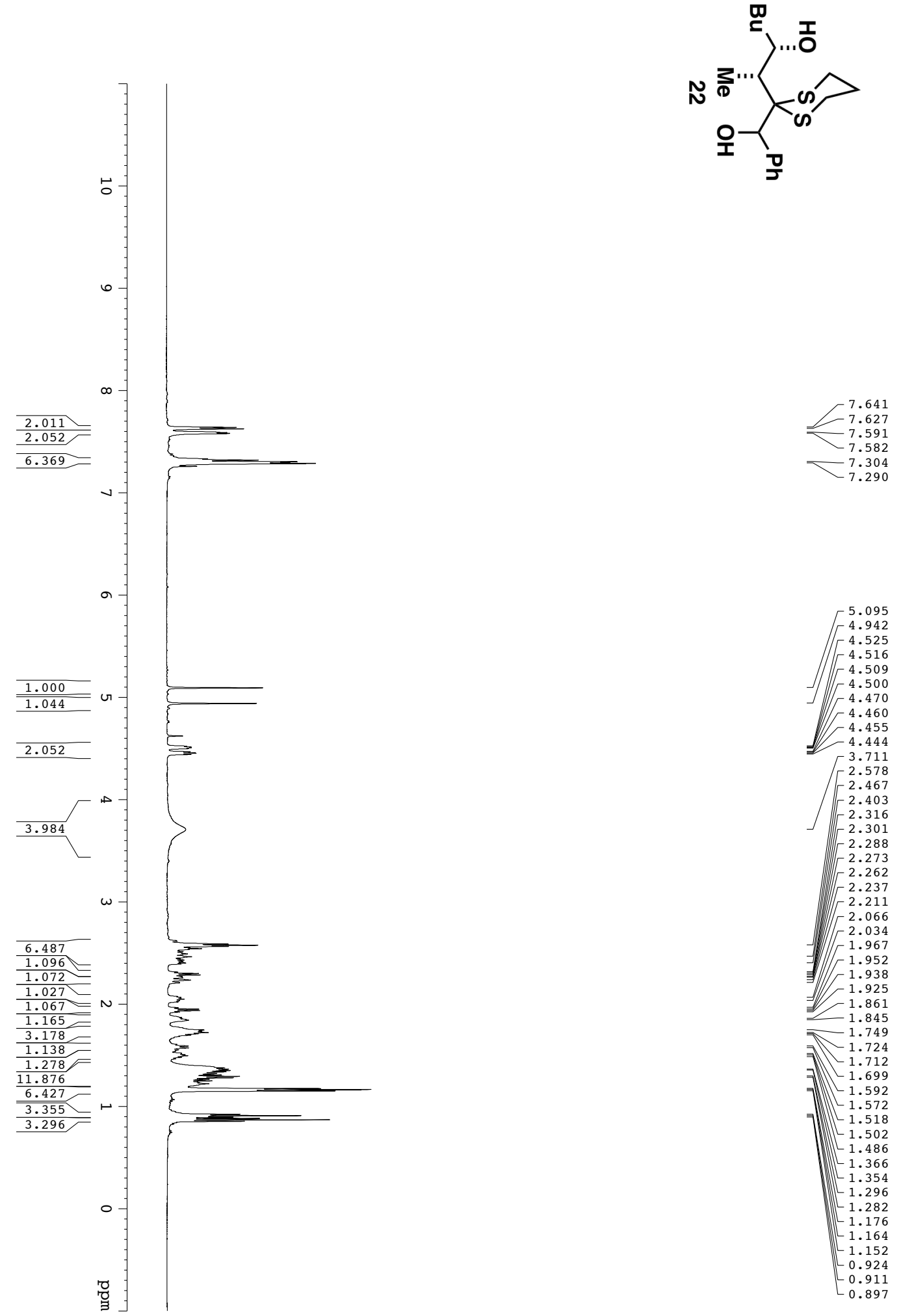

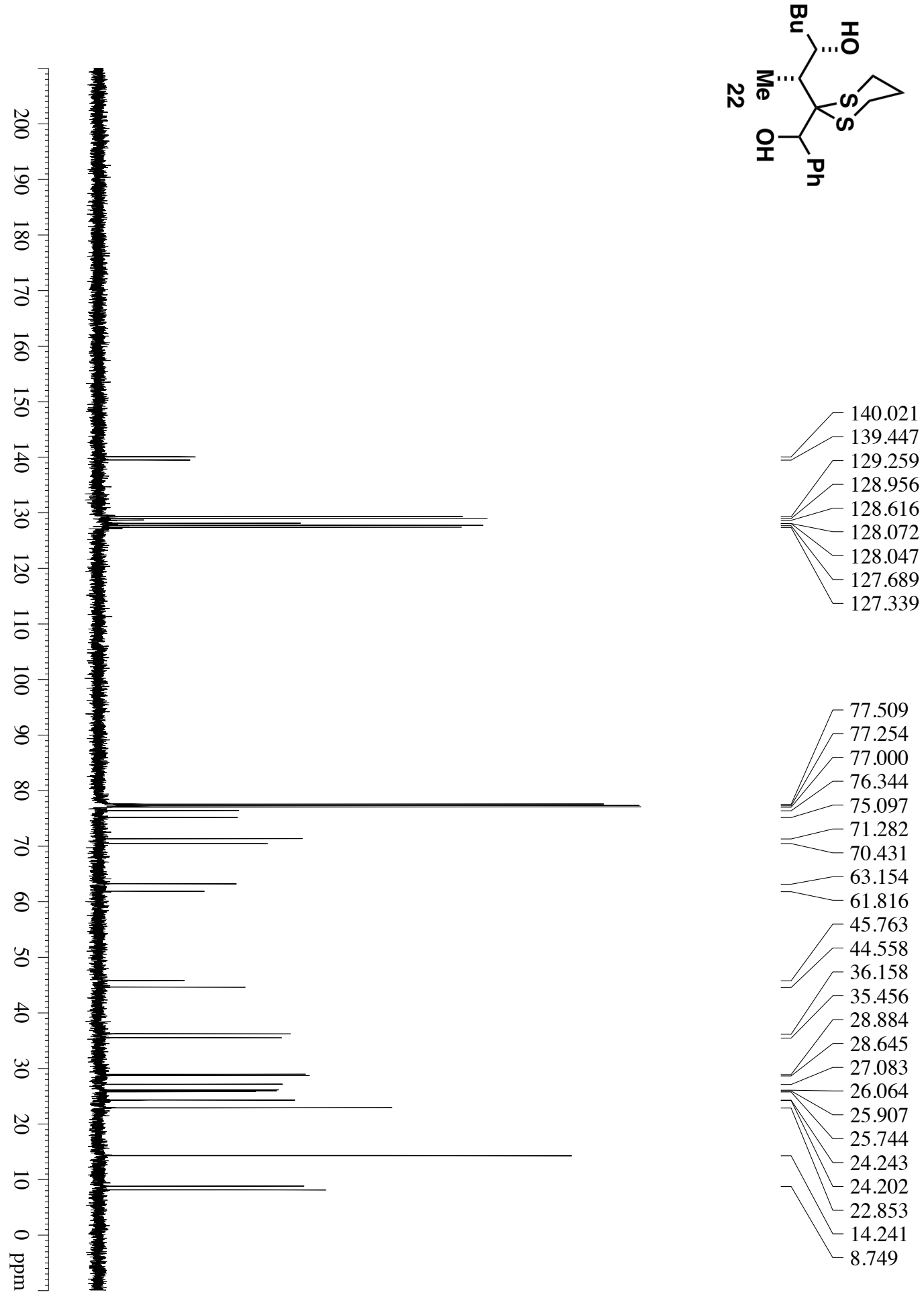

-
$-\quad 6.154$

$-45.763$

44.558
-36.158

$-36.158$

$-35.456$

$-28.884$

$-28.645$

27.083

26.064

$-25.907$

$-25.744$

$-24.243$

$-24.202$

$-22.853$

$-14.24$

$-8.749$ 


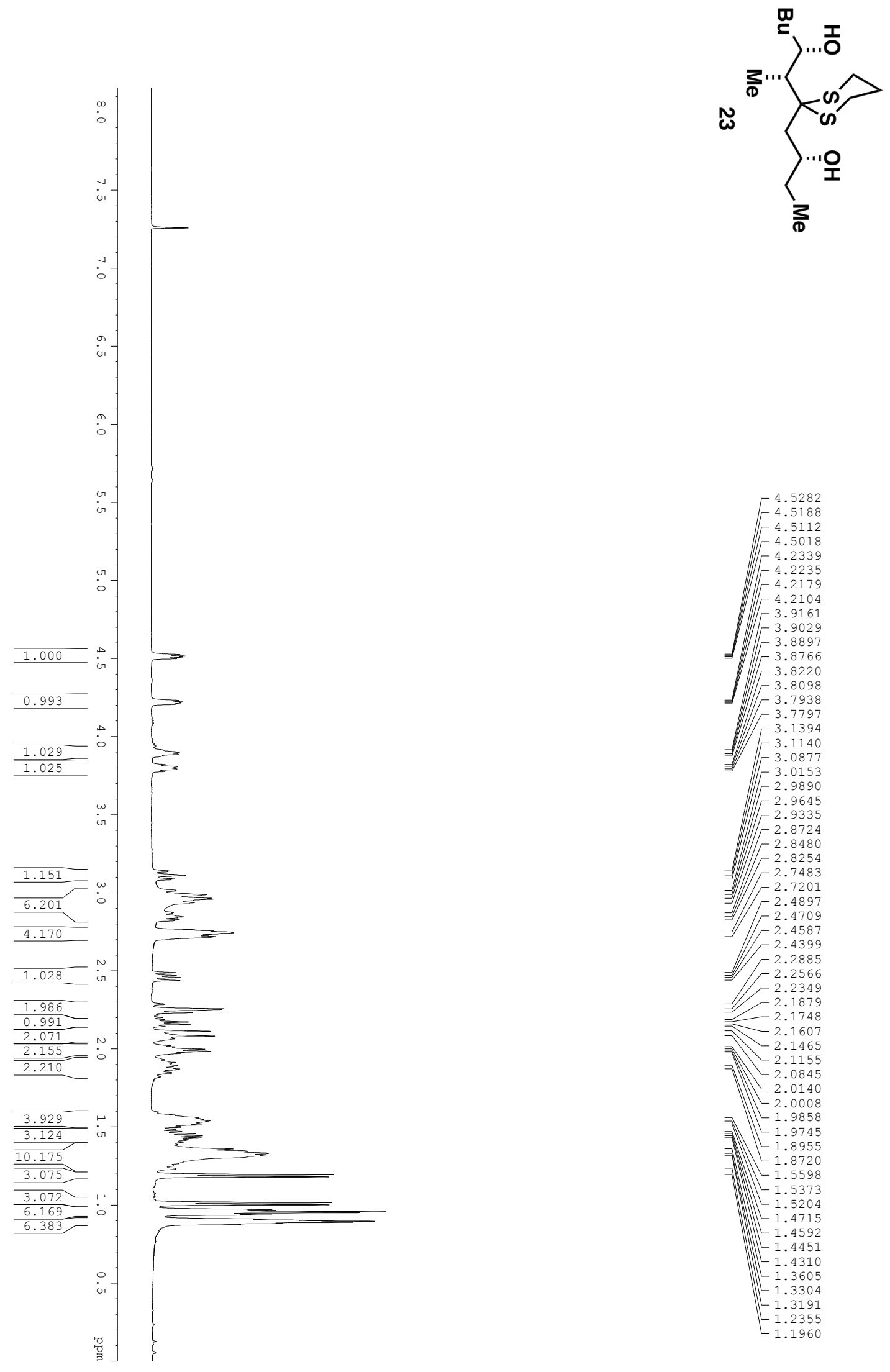



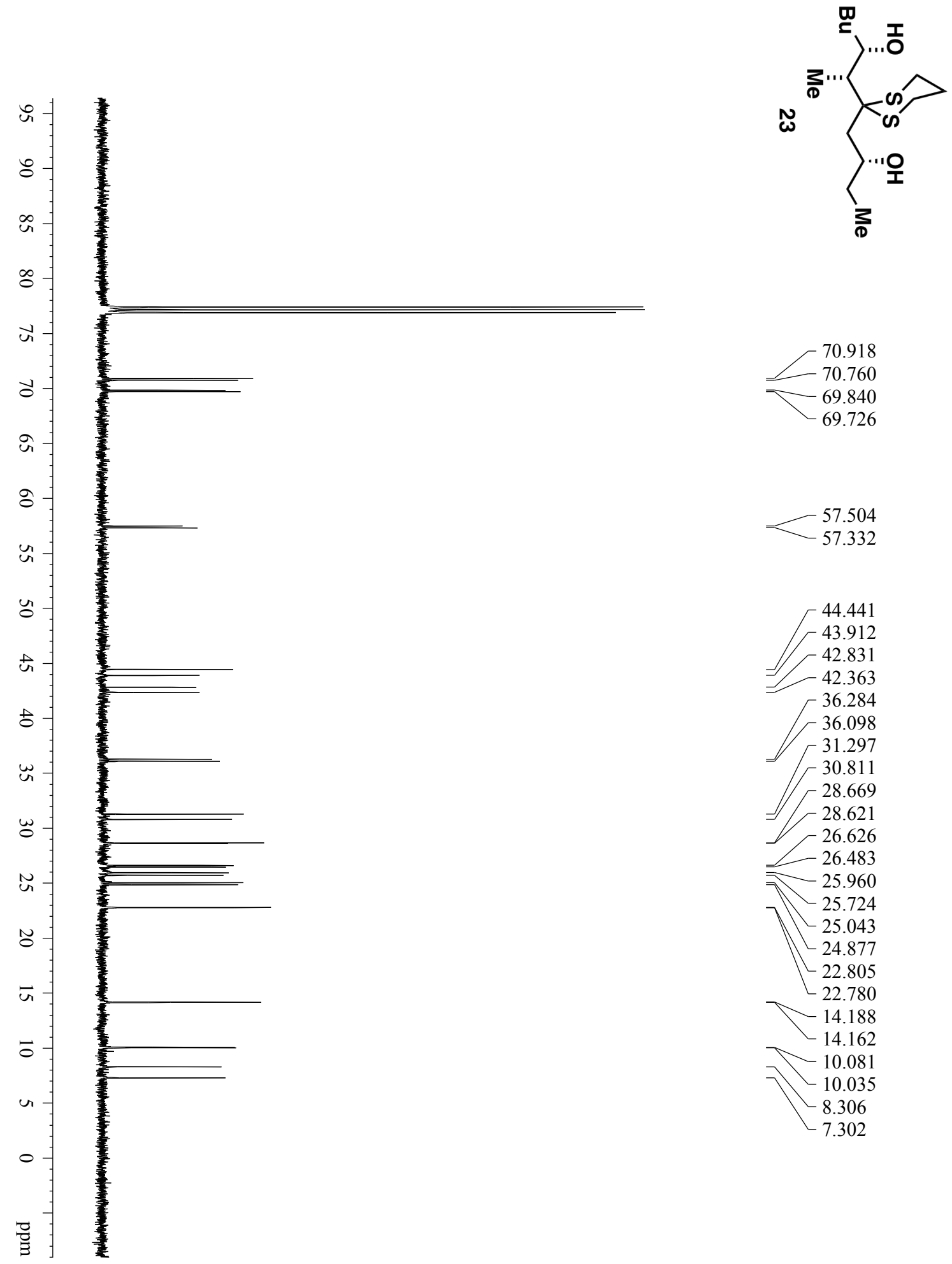

44.441
43.912
$-\begin{array}{r}42.831 \\ 42.363 \\ -36.284\end{array}$

$\Gamma^{36.284}$

31.297
-30.811

$-30.811$

$-28.669$

$-28.621$

$-26.626$

26.483

$-25.960$

$-25.724$

$\mathbb{} \backslash 25.043$

$-24.877$

$-22.805$

22.780

$-14.188$

$-14.162$

$-10.081$

10.035

$-8.306$

7.302 


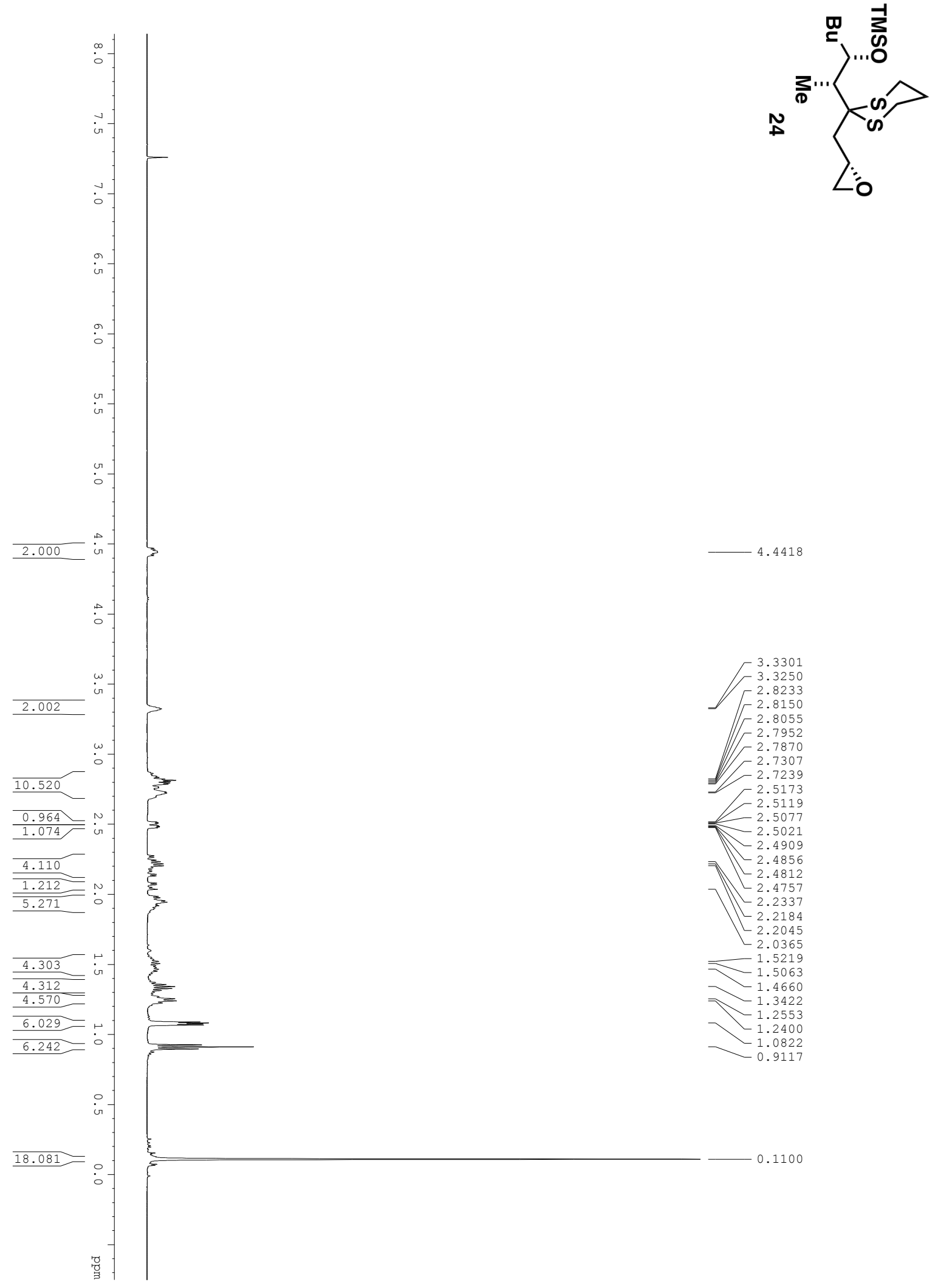



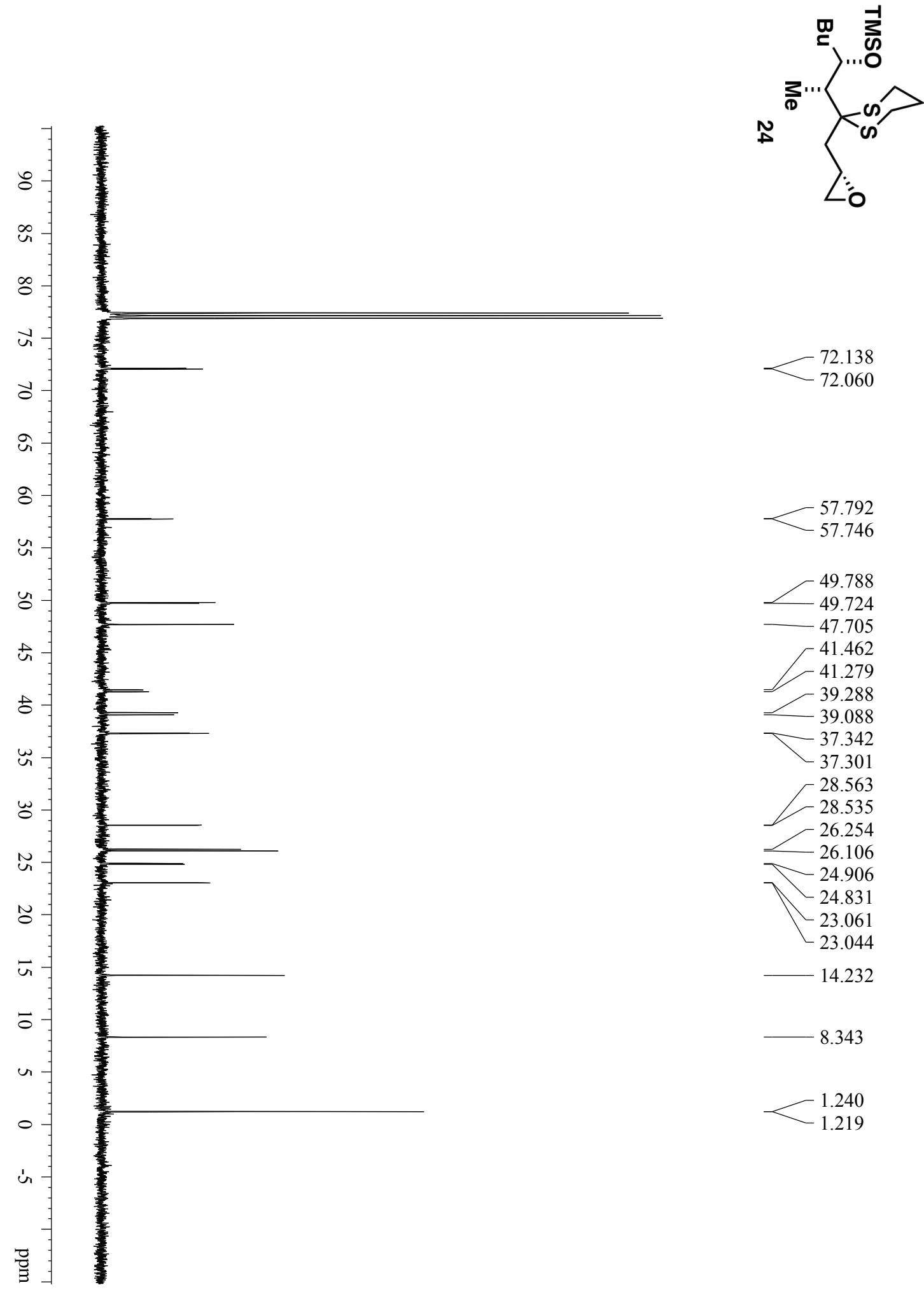

47.705

41.462

$-41.279$

$-39.288$

$\begin{array}{r}-39.088 \\ \hline\end{array}$

× 37.342

$-37.301$

28.563

$-28.535$

26.254

26.106

$-24.906$

24.831

$-23.061$

$-23.044$

$-14.232$

8.343

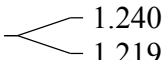



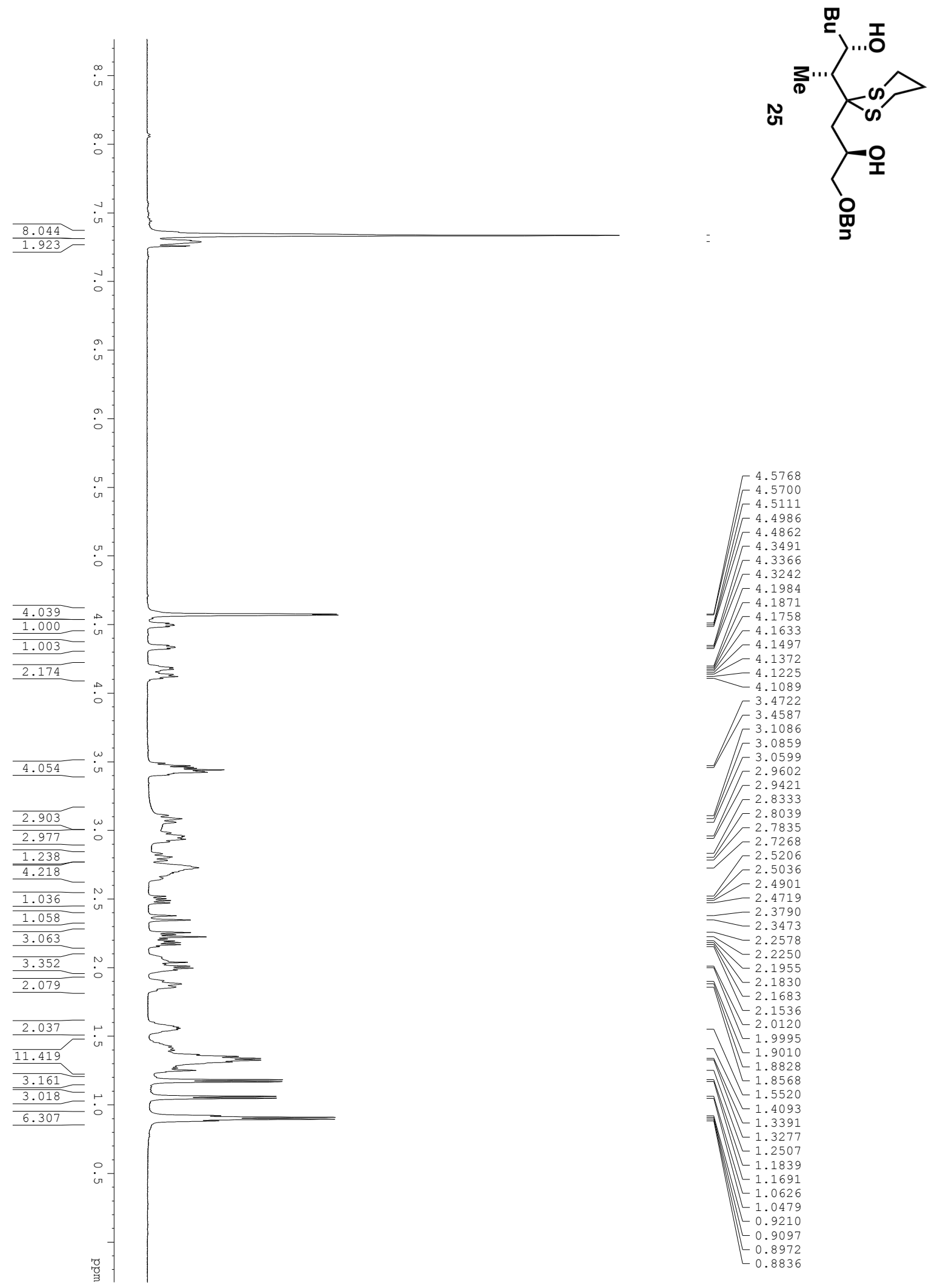

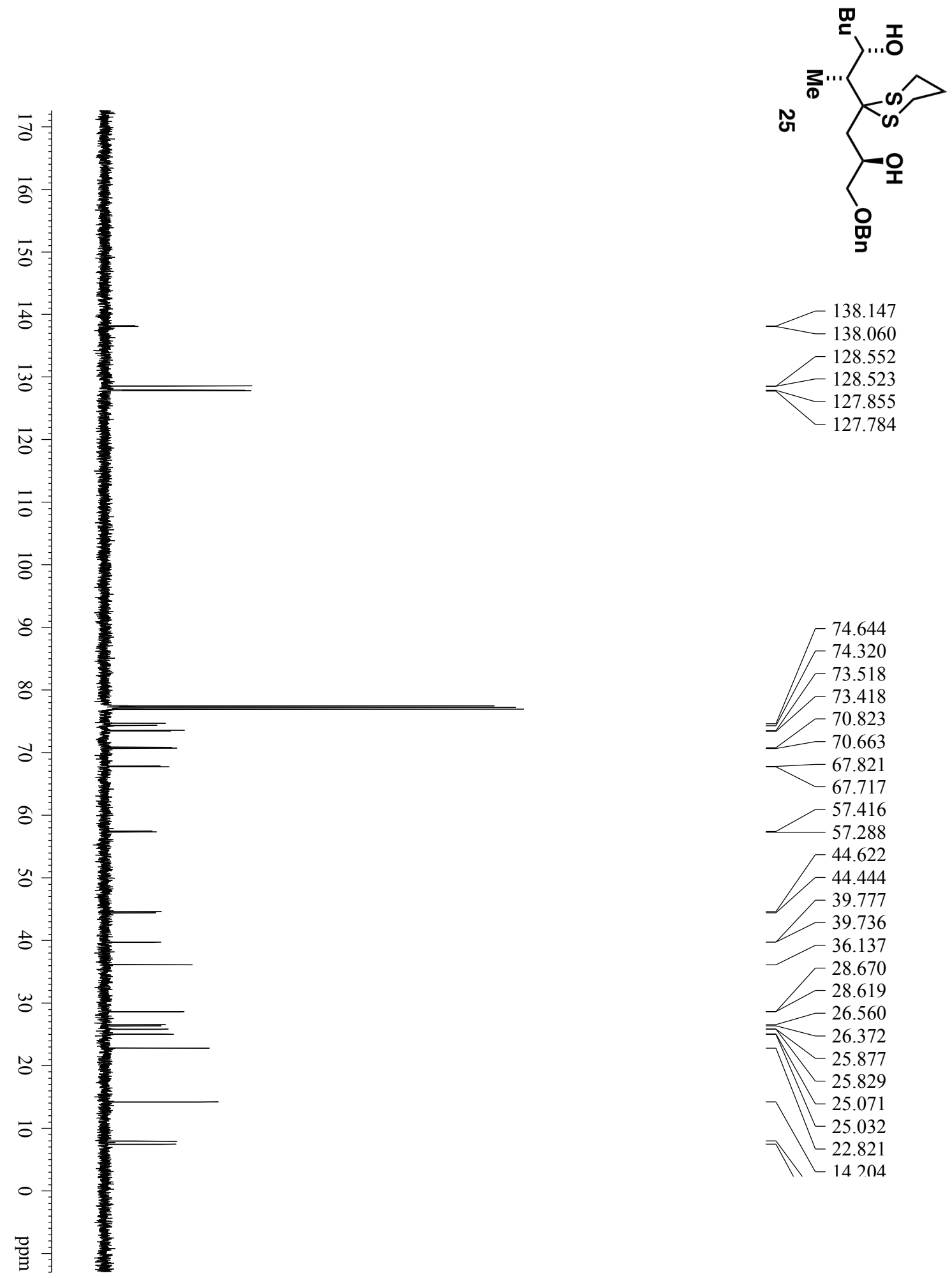
44.622

$-44.444$

$-39.777$

$-39.736$

$-36.137$ 28.670 28.619

$-26.560$

$-26.372$

$-25.877$

$-25.829$

$7-25.071$

$-25.032$

$\sqrt{-22.821}$

$-14.204$ 


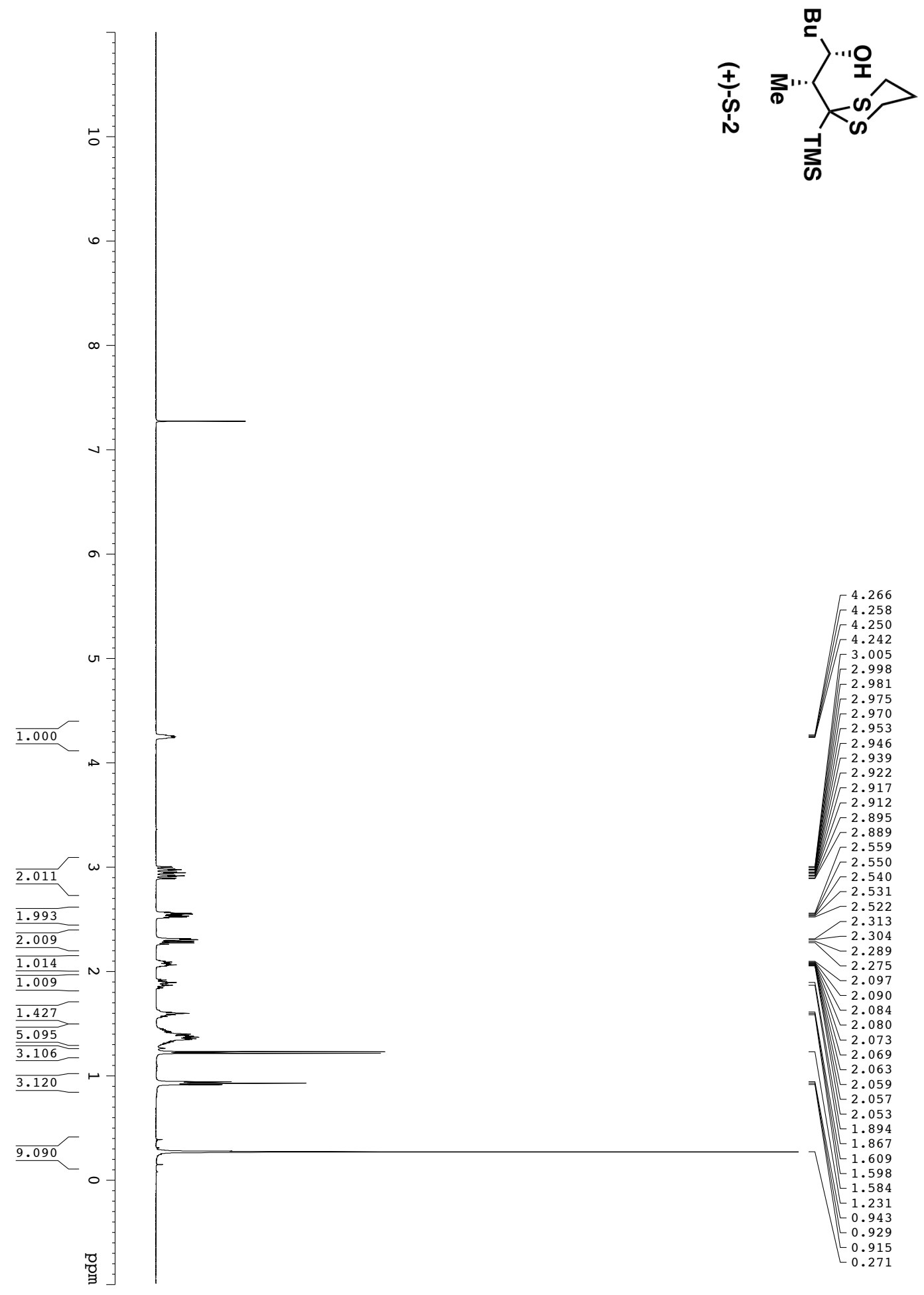



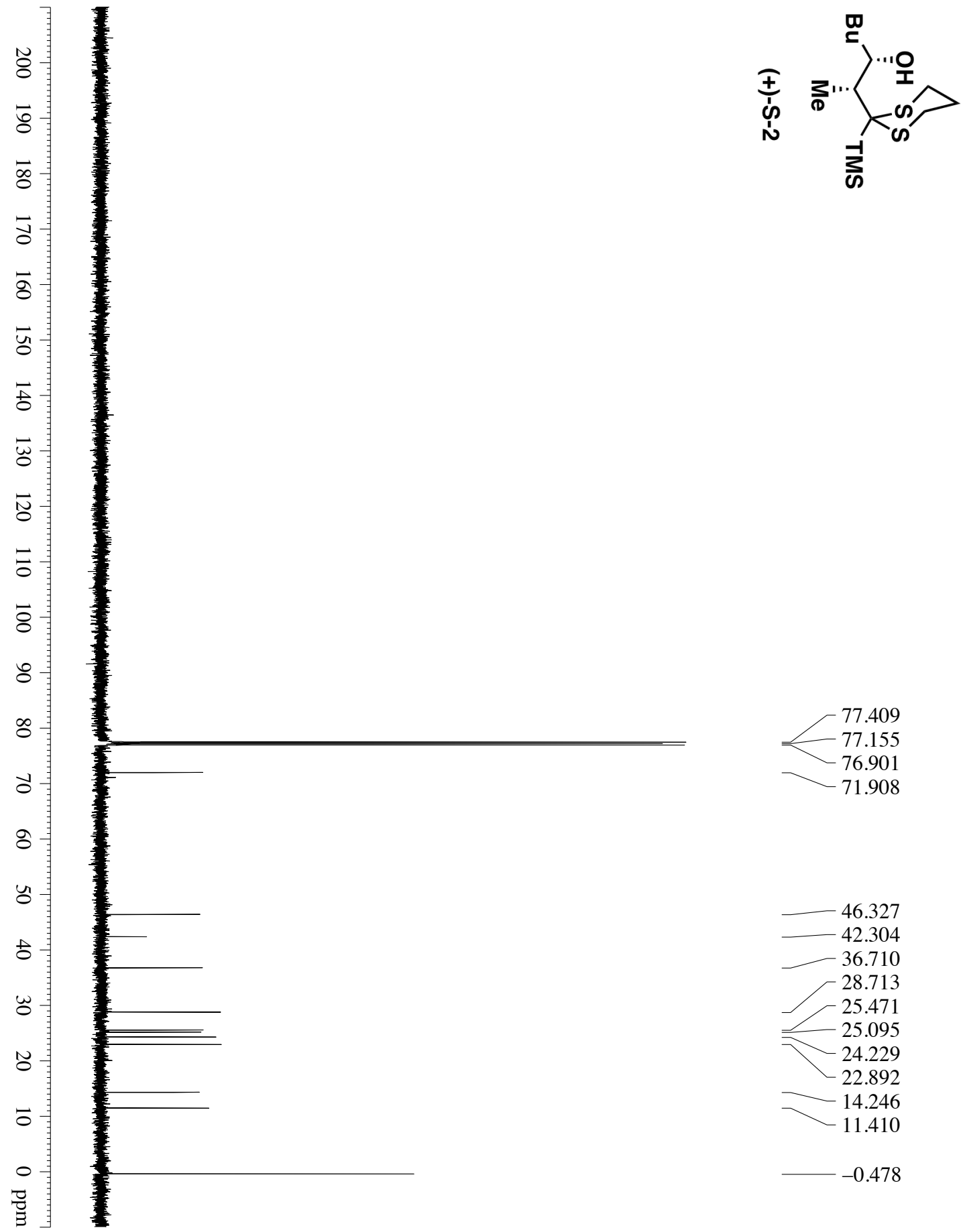

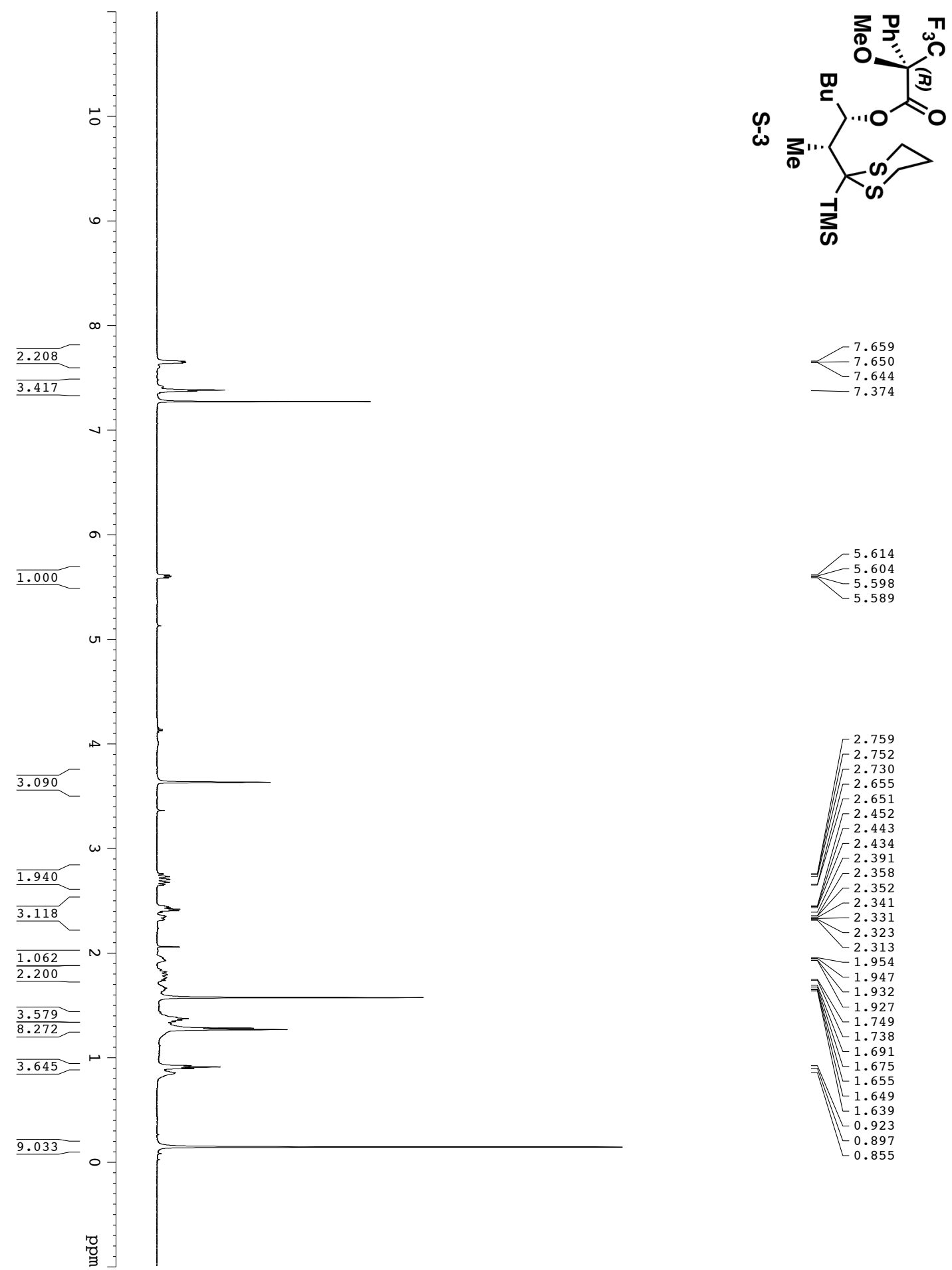

S-51 


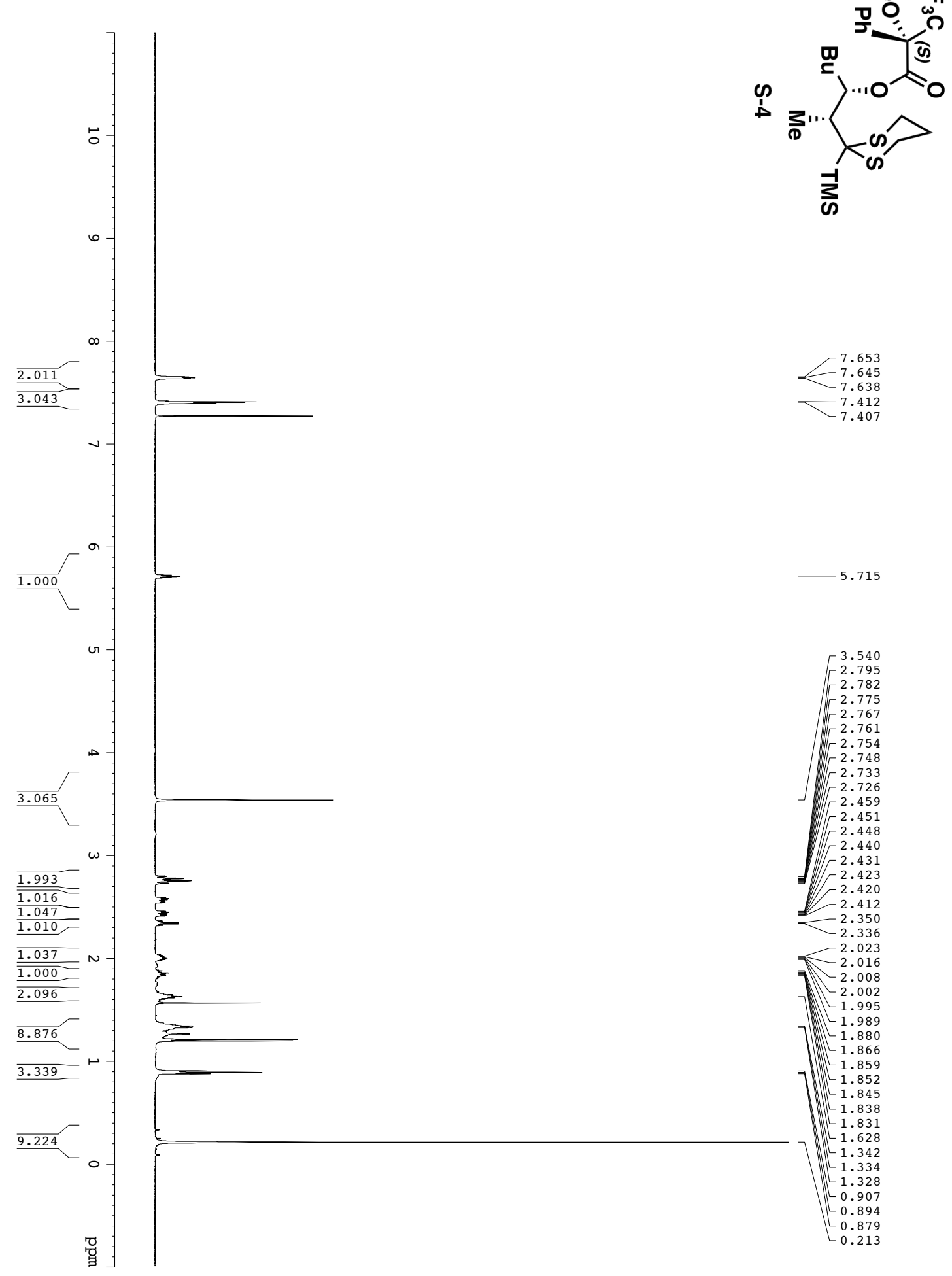




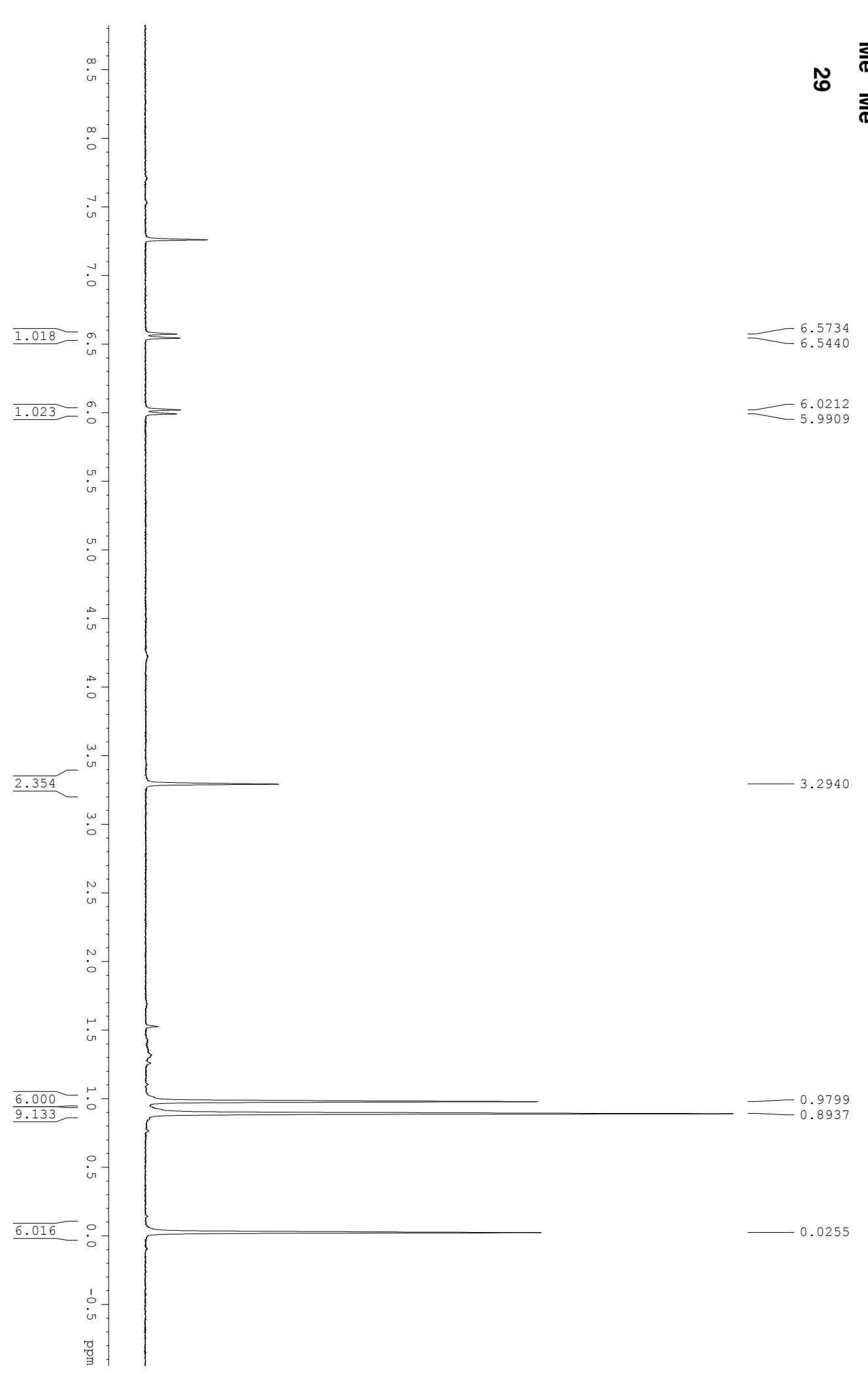



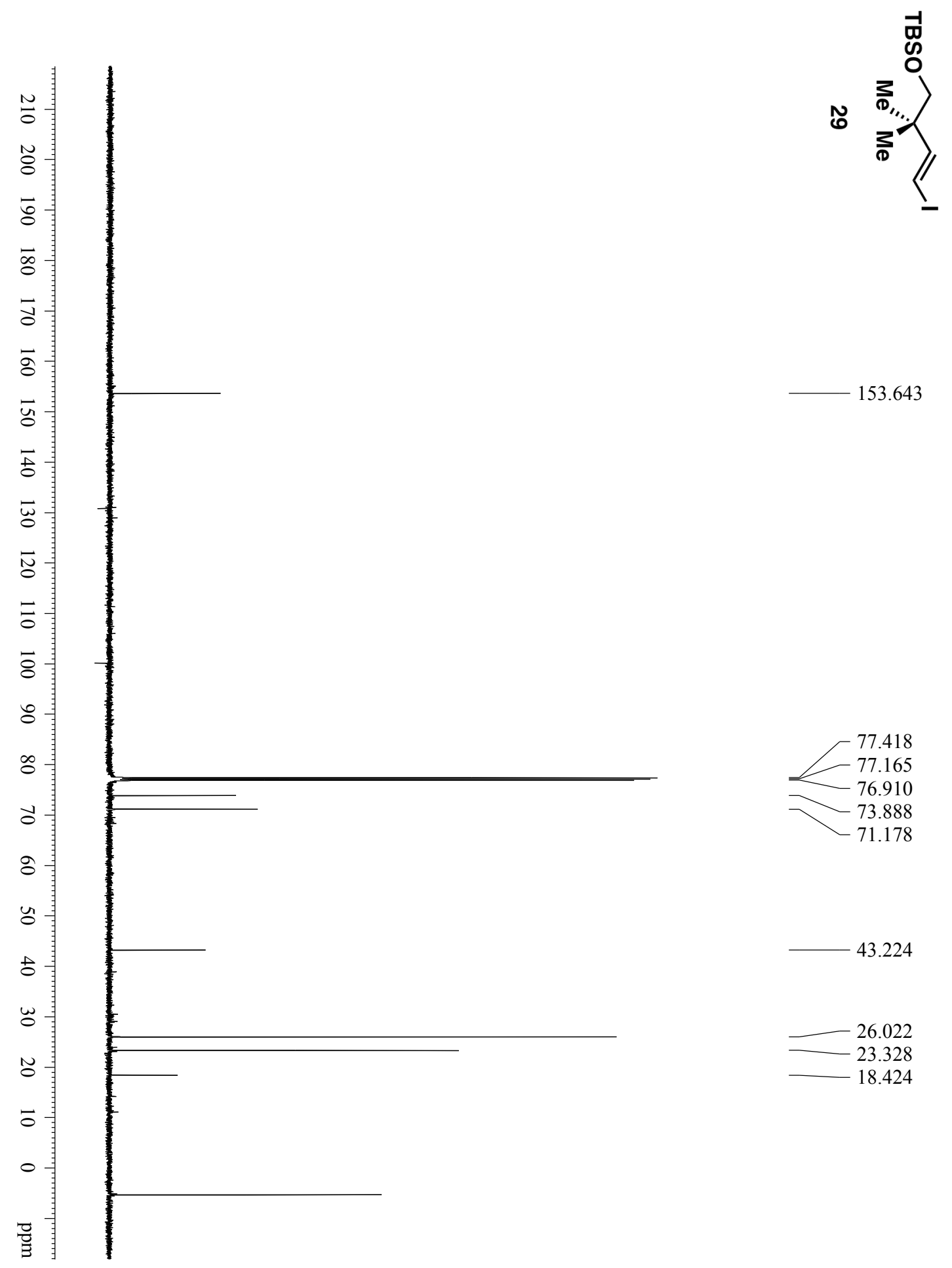

153.643

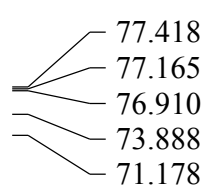

$-43.224$

- 26.022

23.328

$-18.424$ 


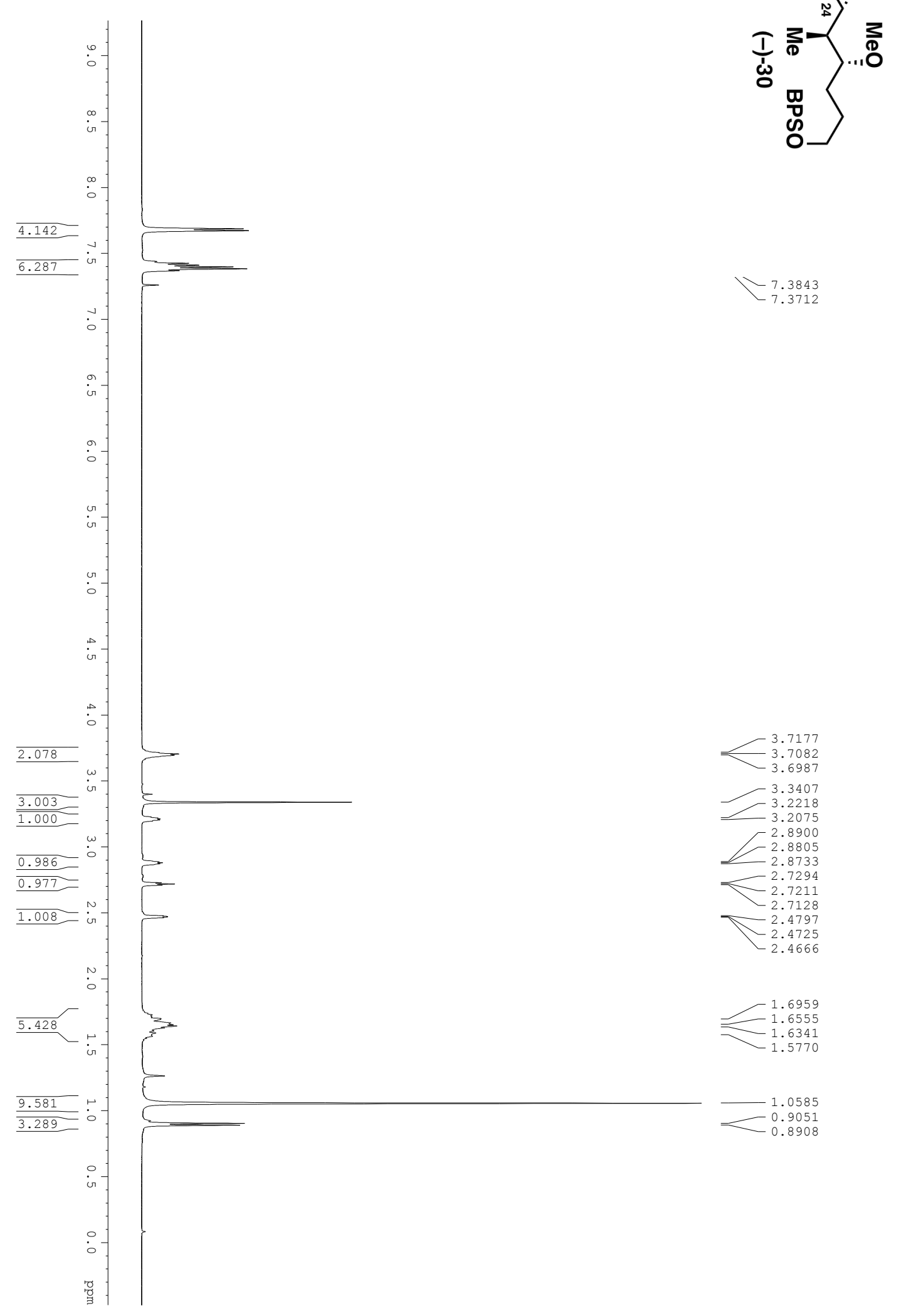

S-55 

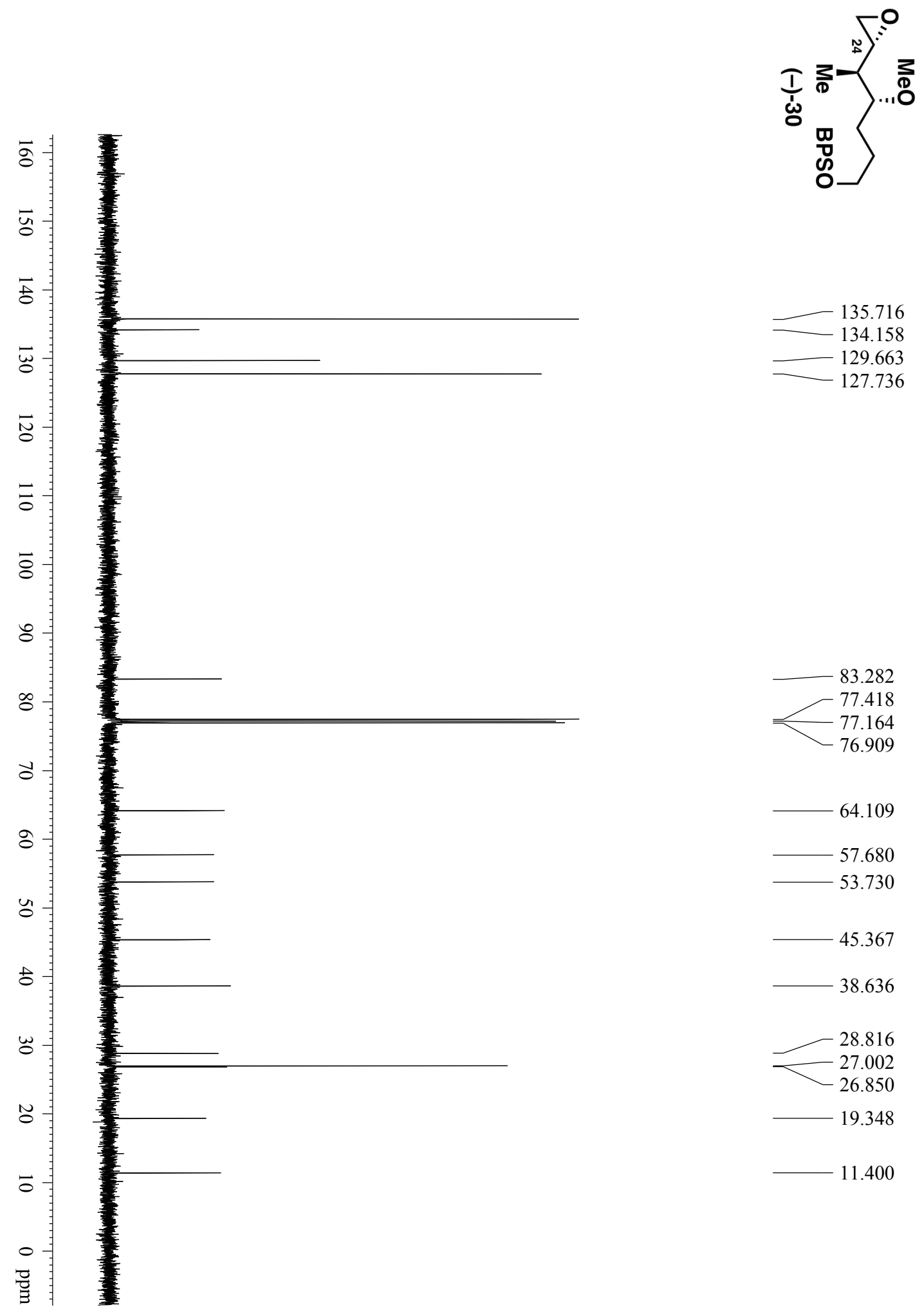

64.109

57.680

53.730

45.367

38.636

$-28.816$

27.002

26.850

19.348

11.400 


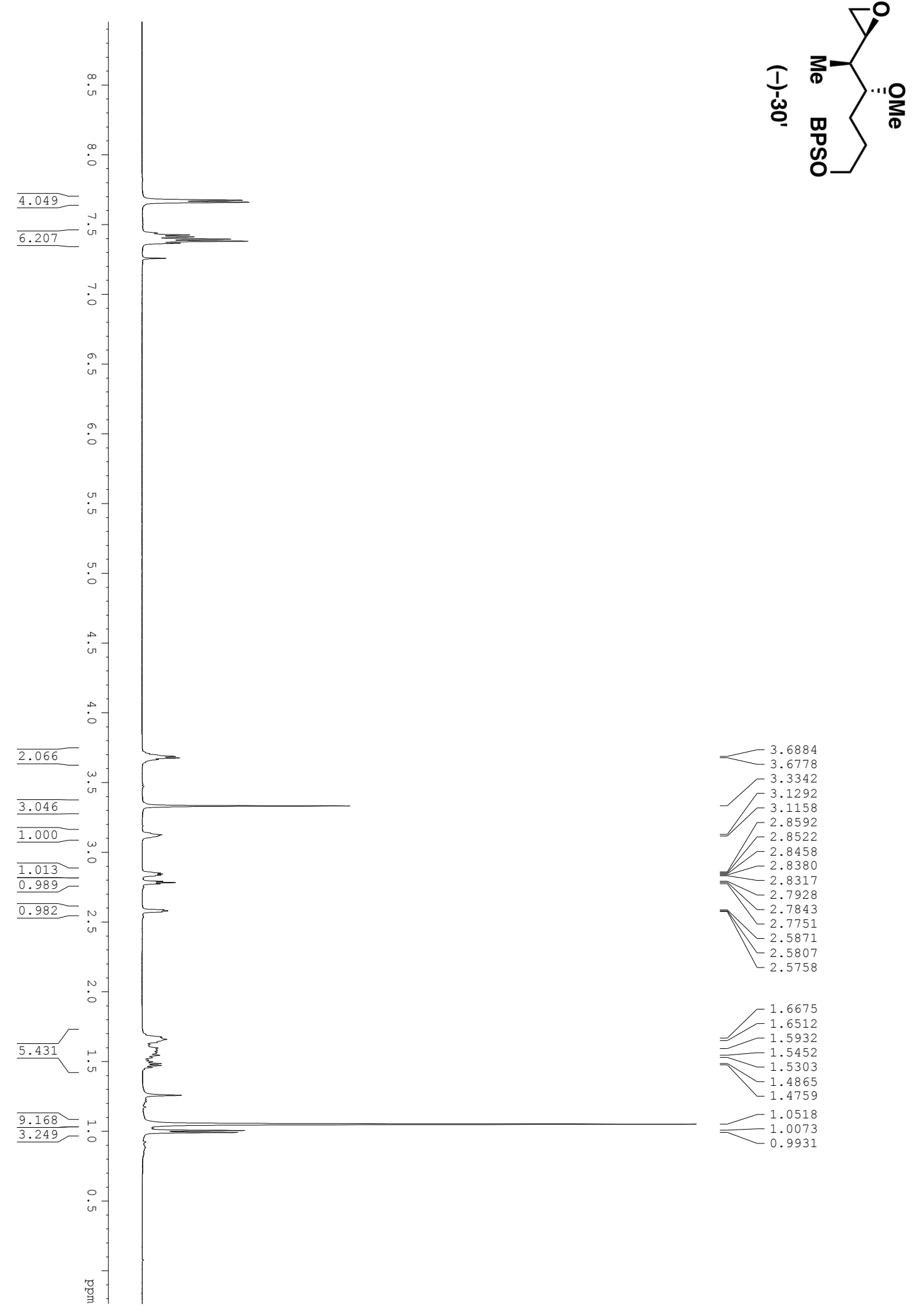



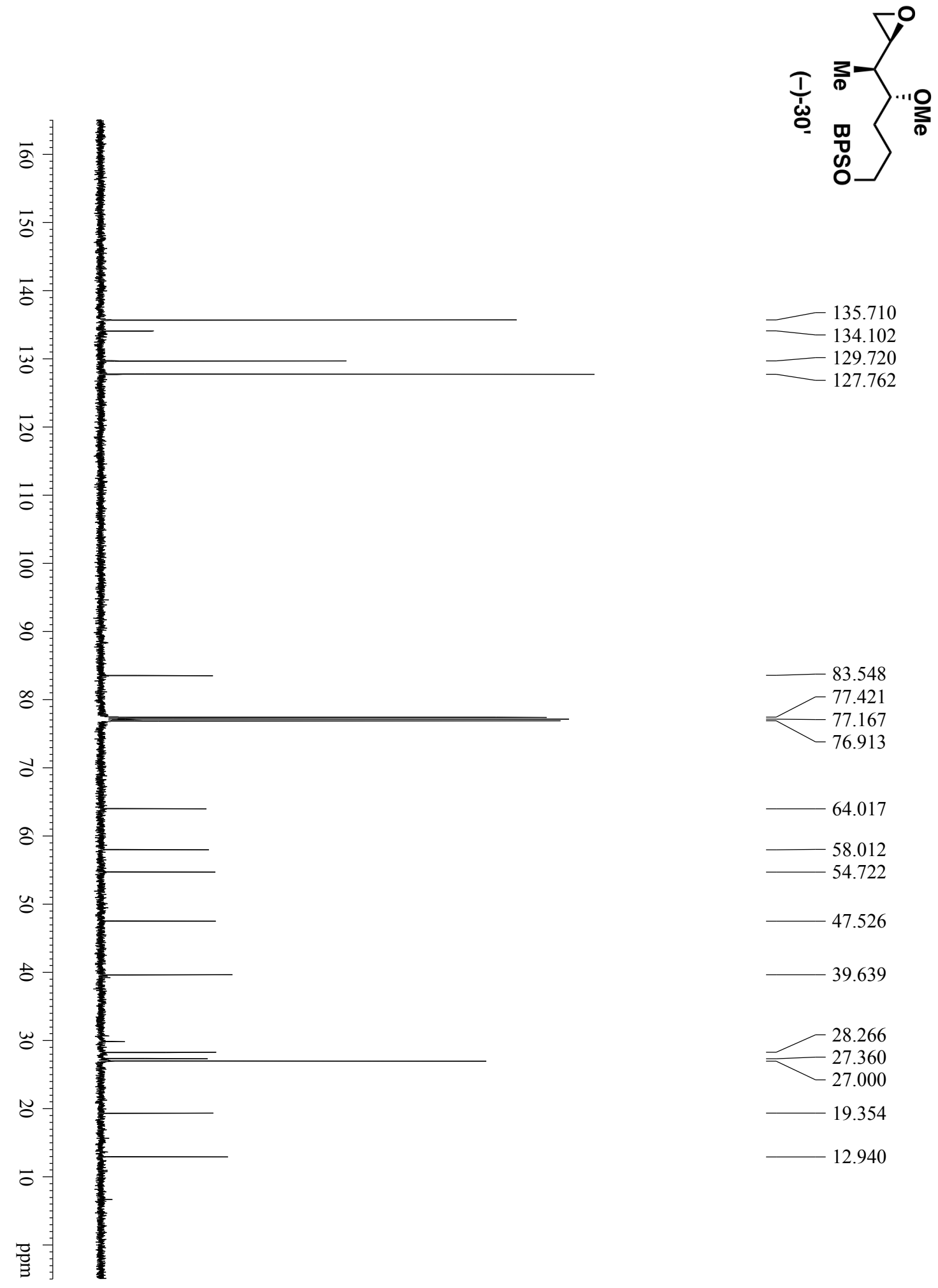

135.710

134.102

129.720

127.762

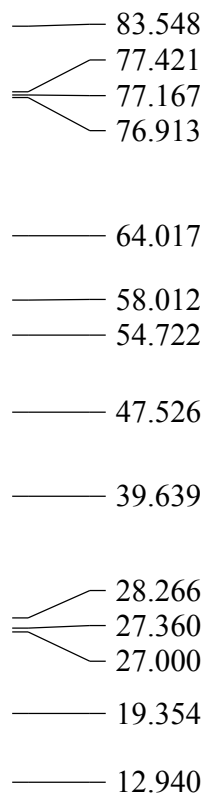




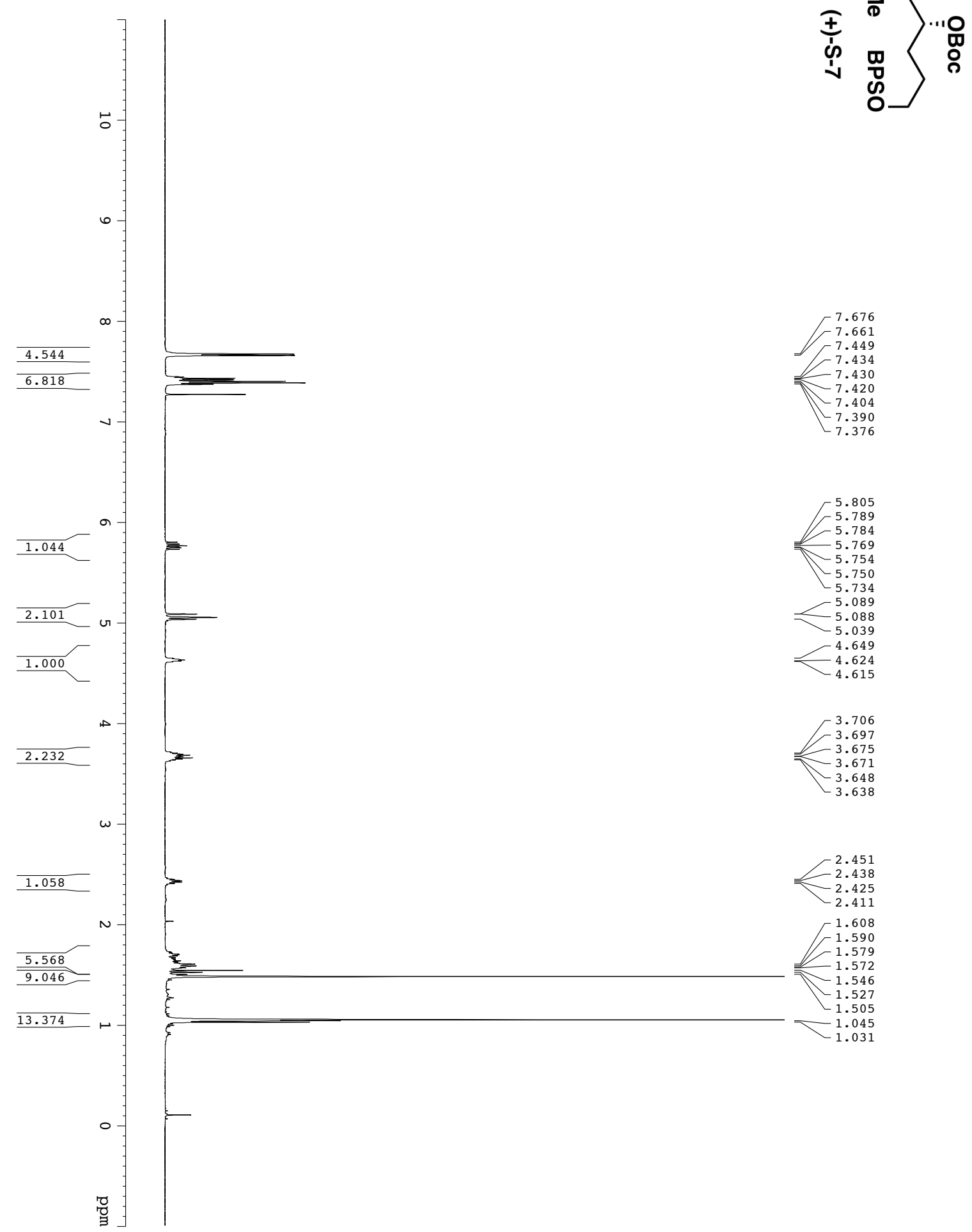




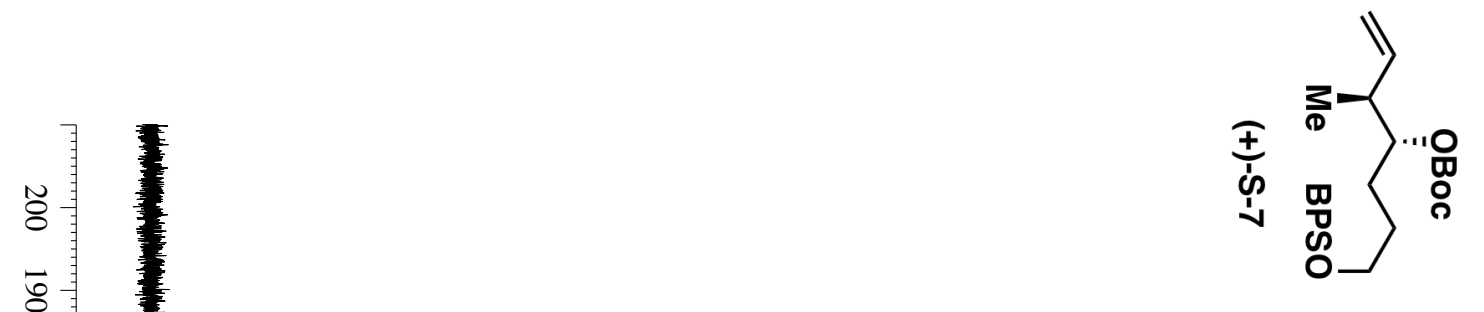

\section{à \\ 8}

\&

tr

t5

फ

్.

$\bar{\sigma}$

8

๖

$\infty$

이

8

y

$b$

w

ำ

○

旁目

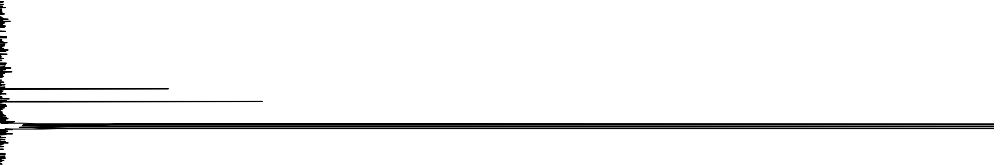

$-81.673$

$-80.127$

$-77.409$

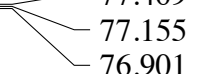

$-63.638$

41.935

$-28.730$

$-27.969$

27.912

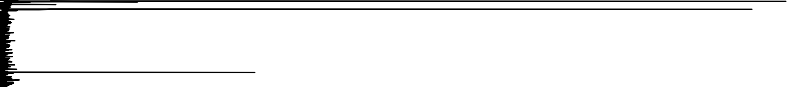

- 26.986

$-19.353$ 


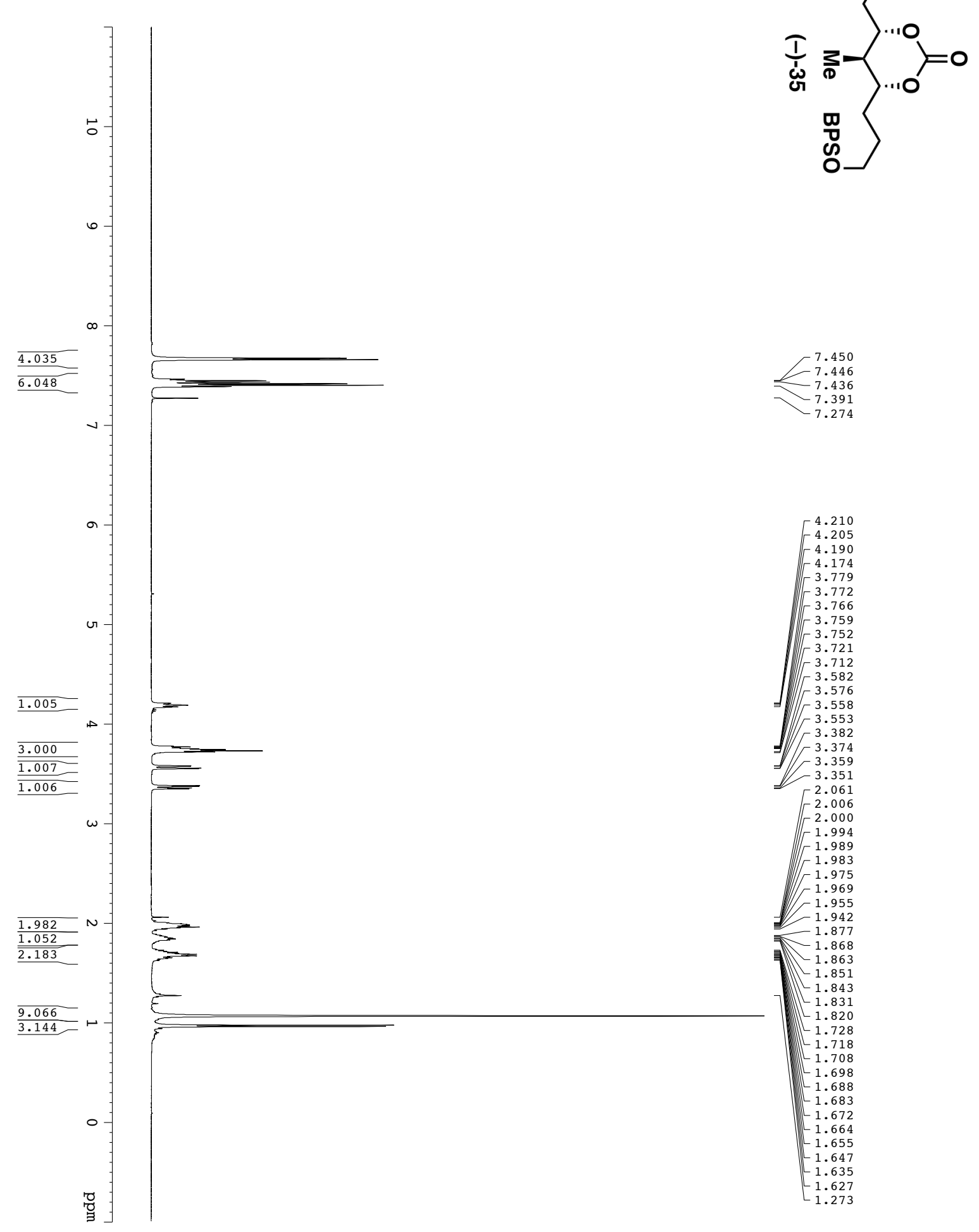



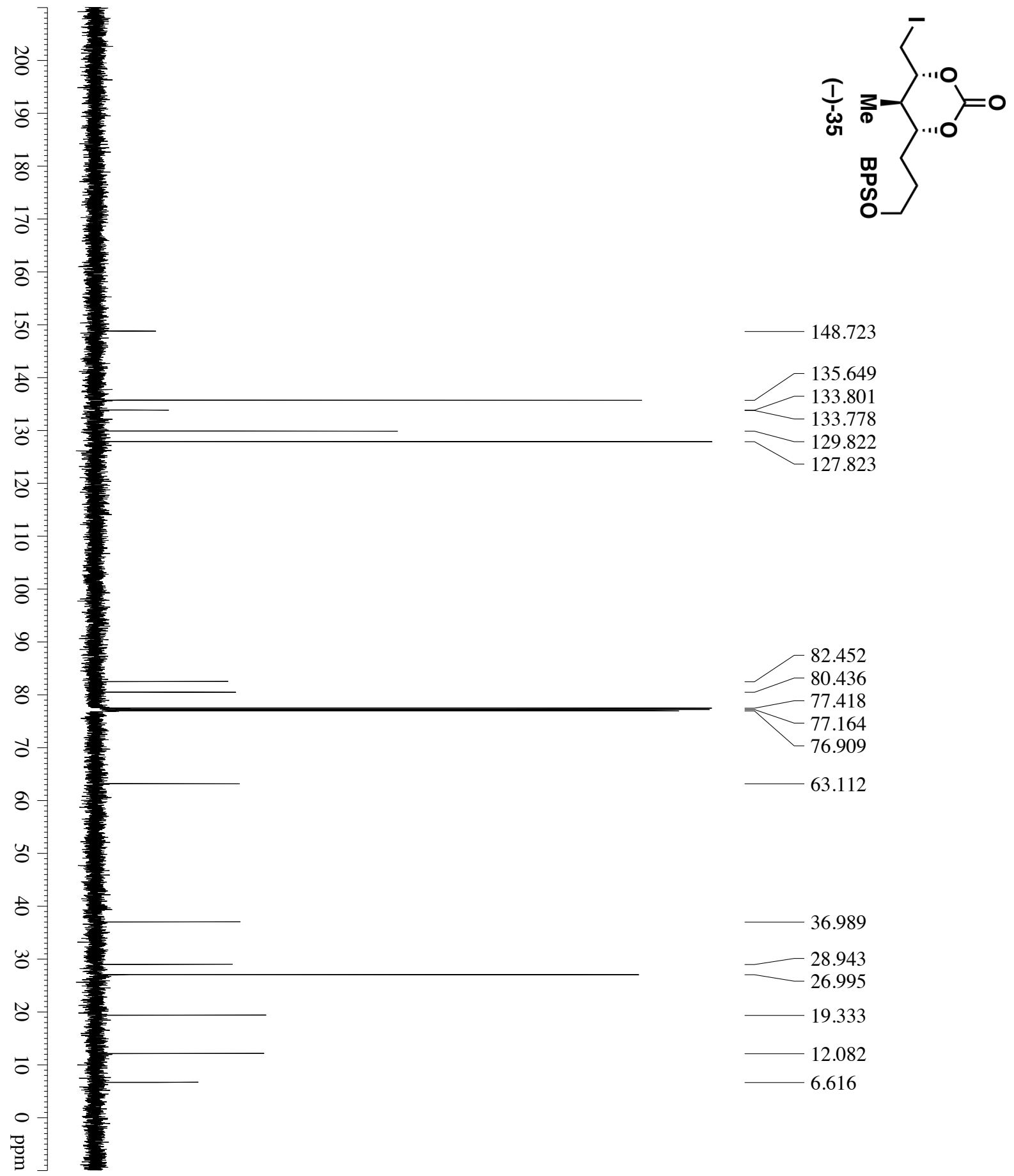

135.649

133.801

$-133.778$

$-129.822$

127.823

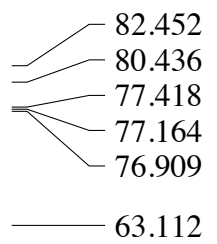

36.989

28.943

26.995

19.333

12.082

6.616 


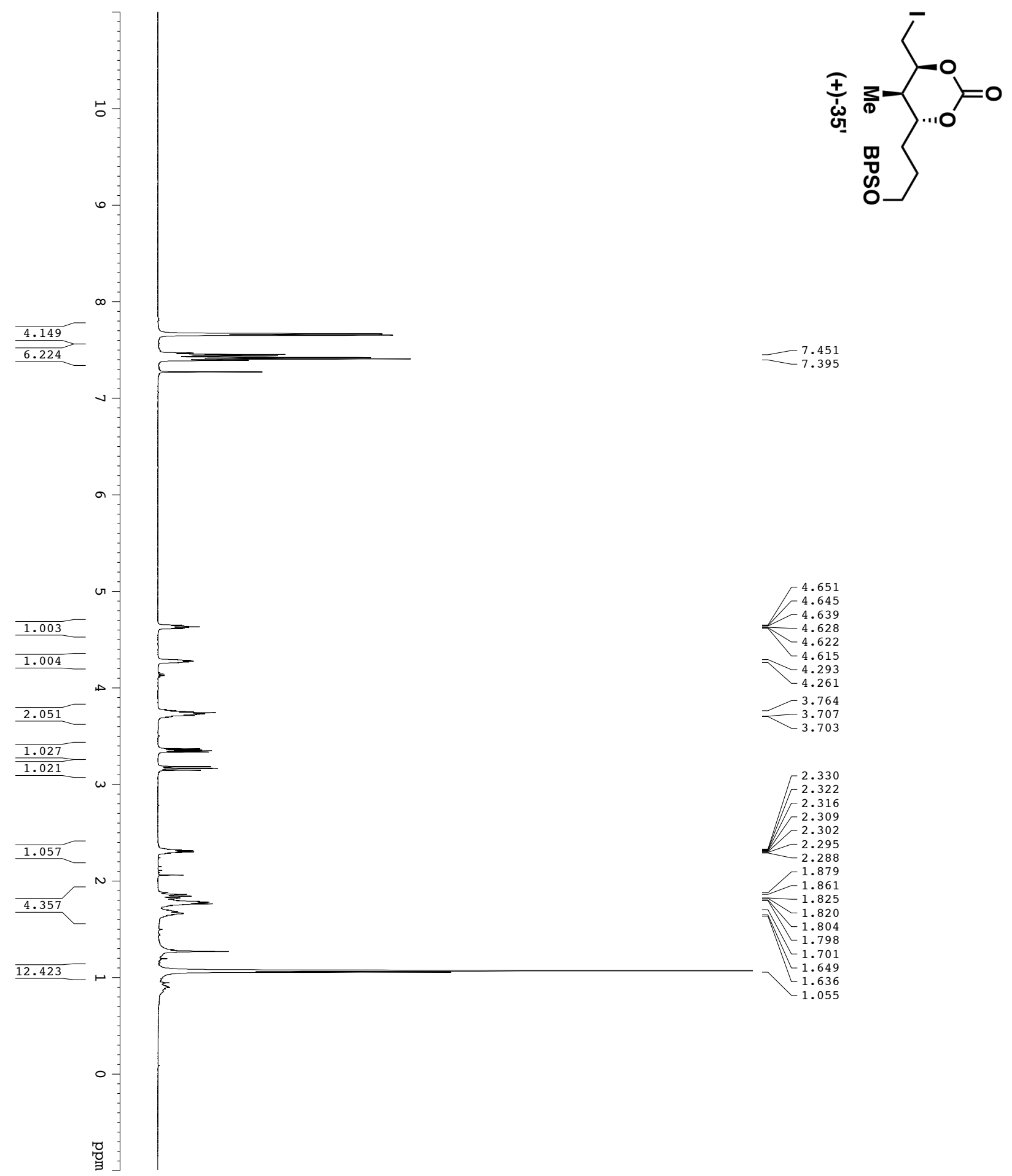




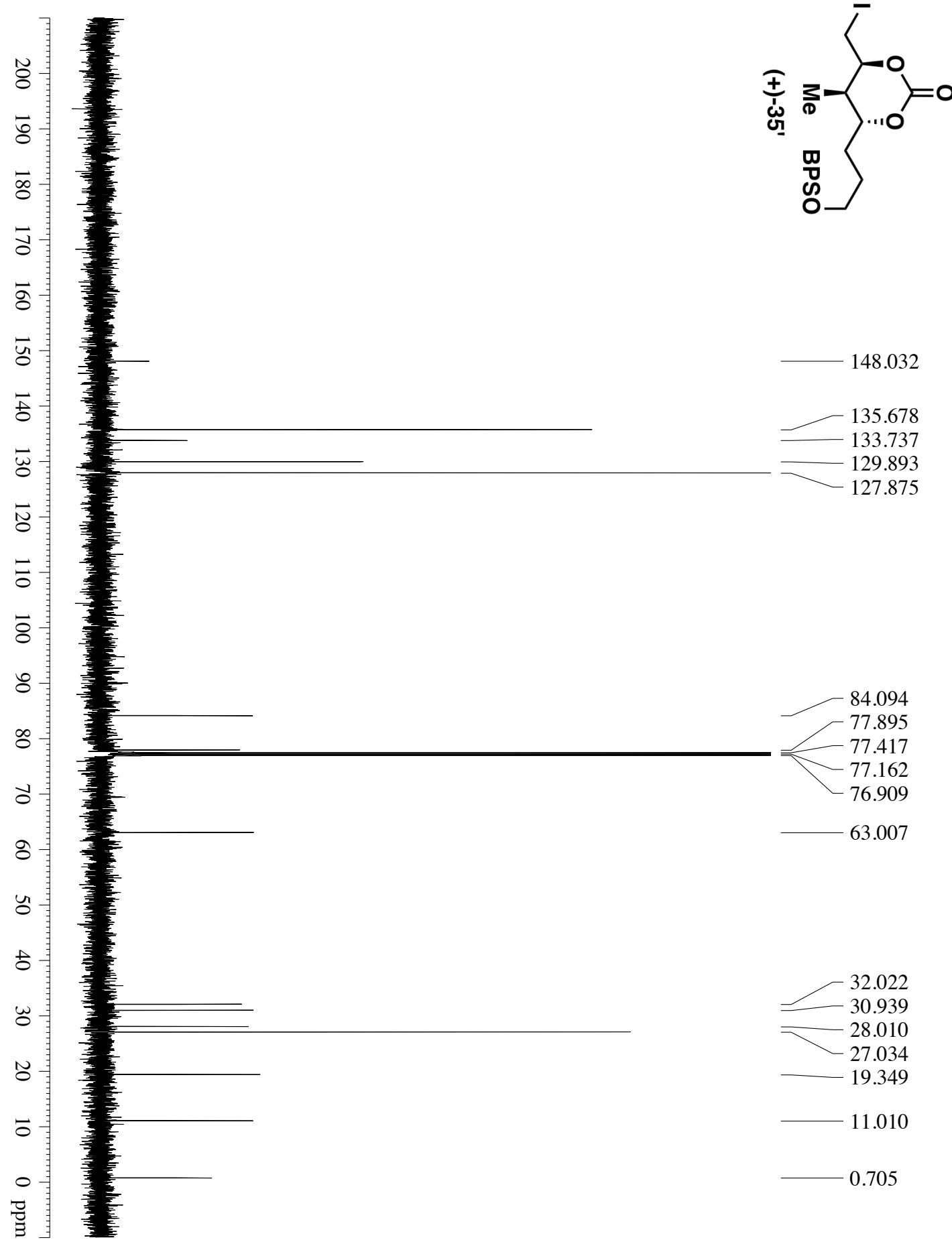




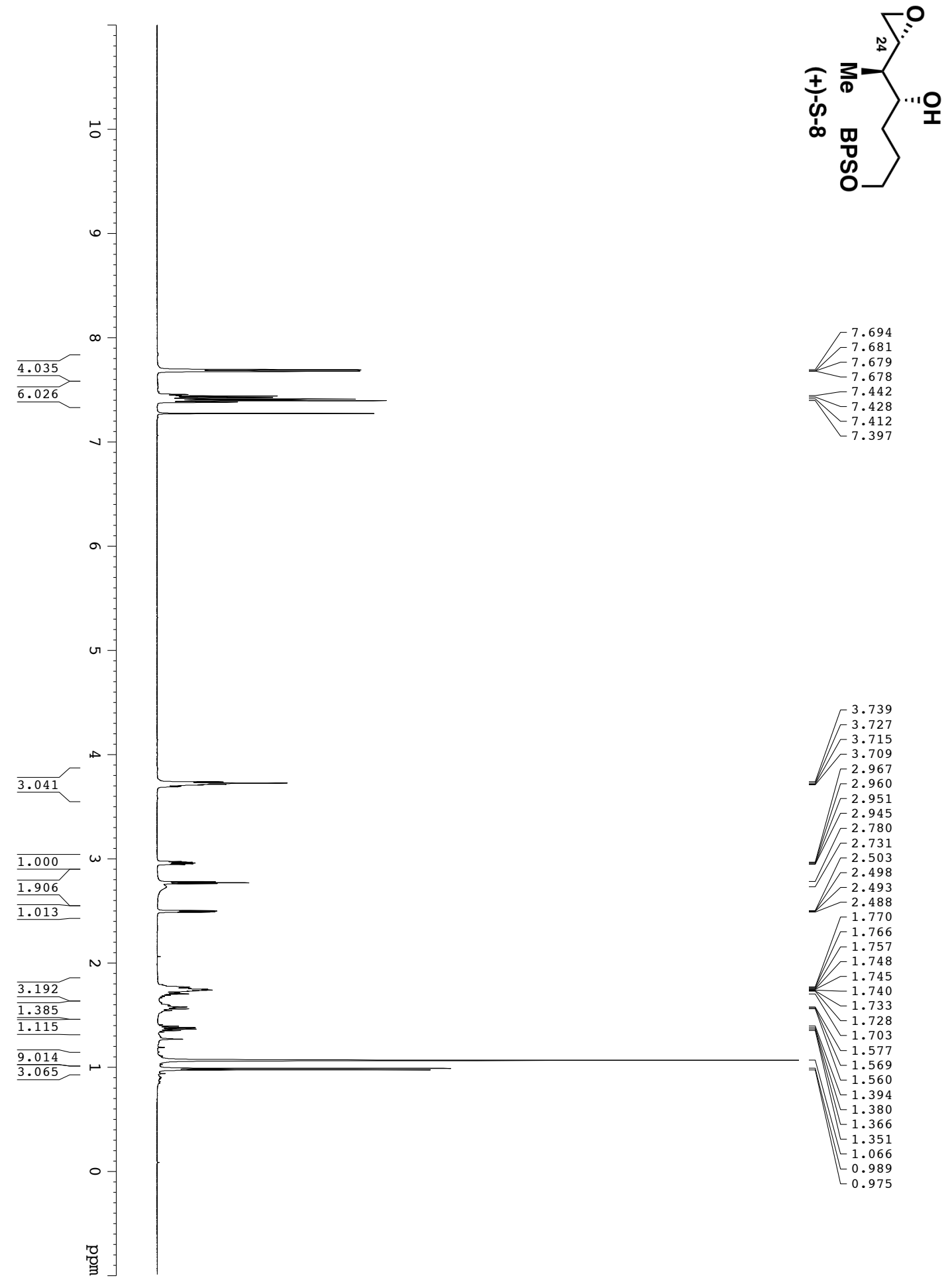




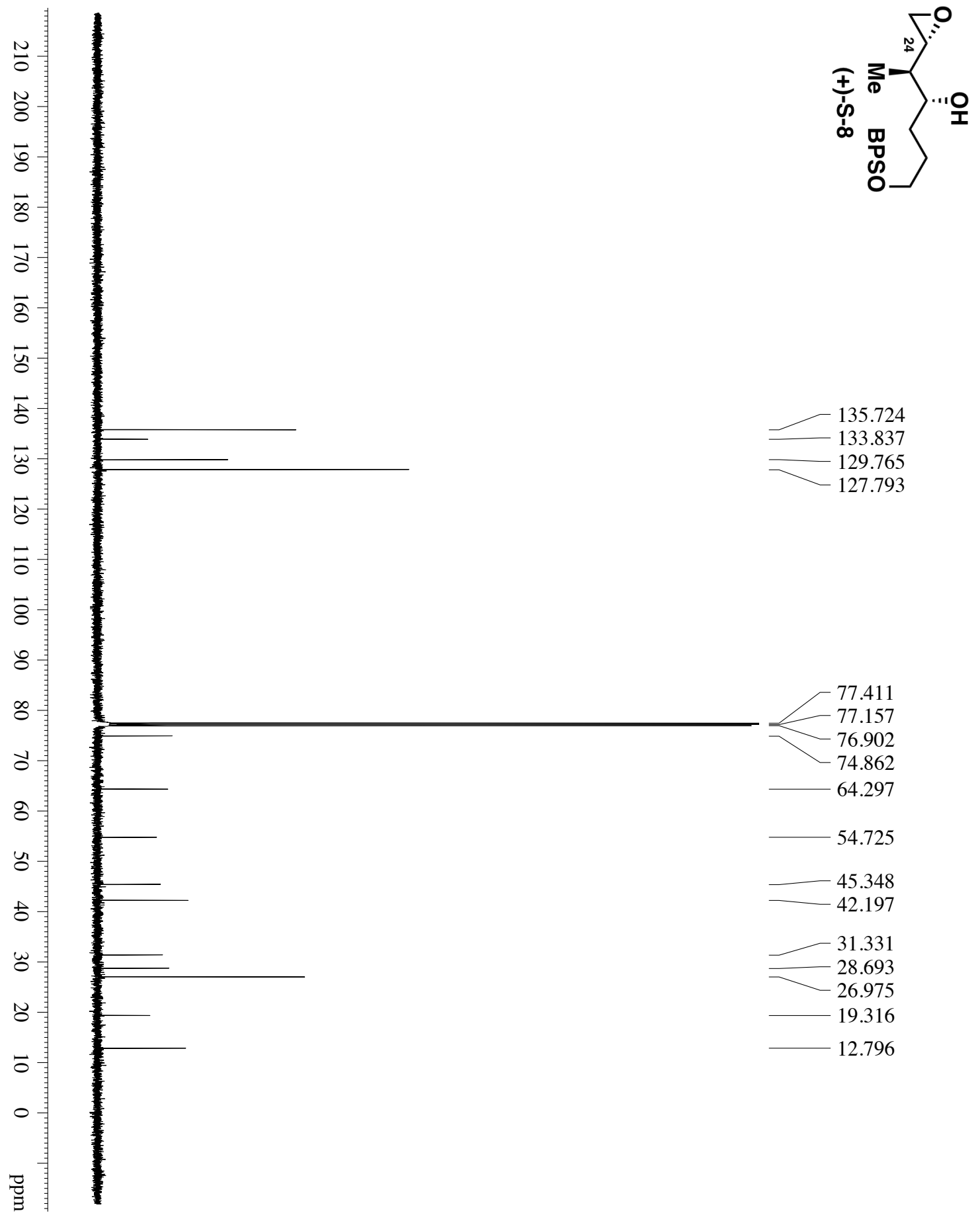




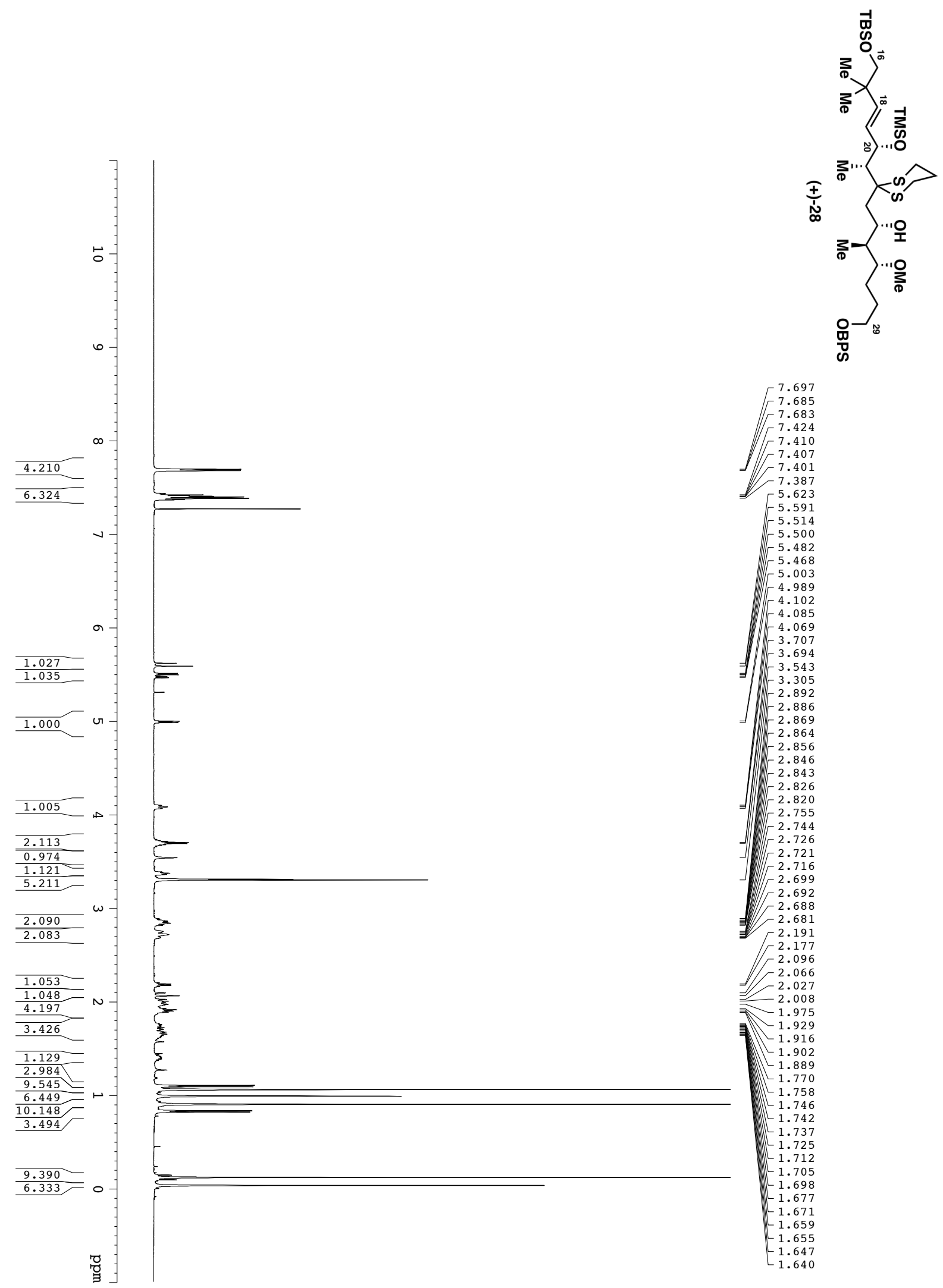




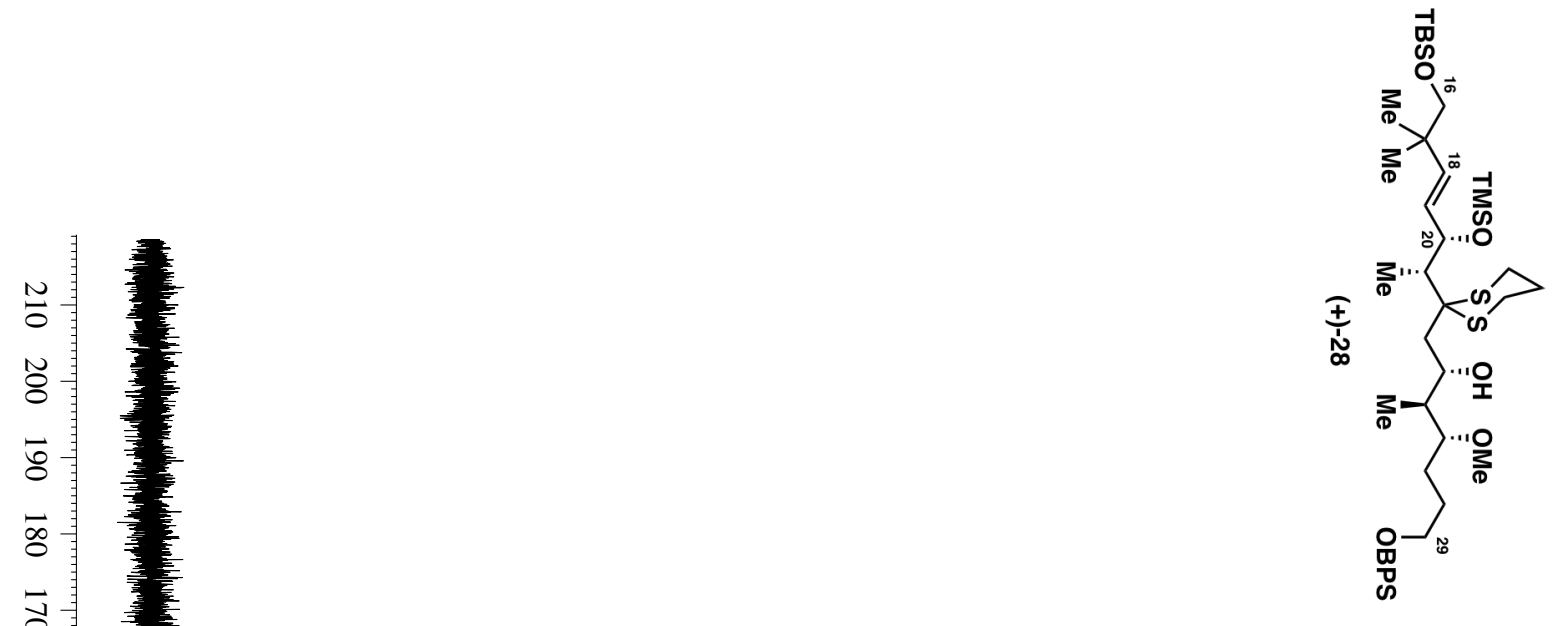

8



$\breve{o}$

동

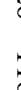

छ

8

8

\&

ठ

8

y

है

c

ำ

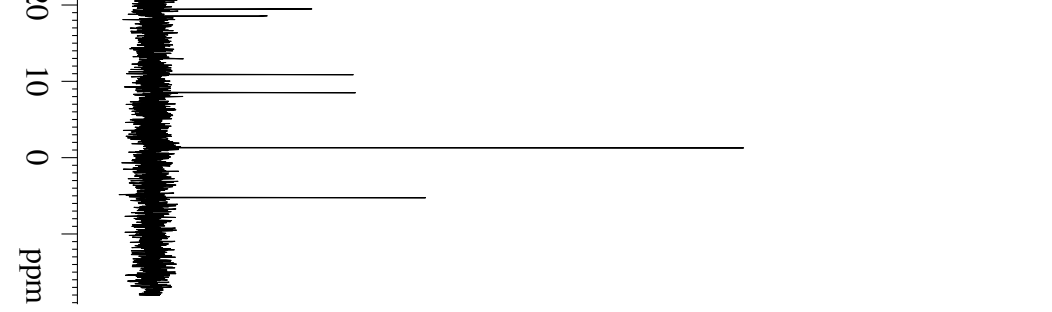

$-137.863$

81.591

$-77.398$

$-77.144$

$-76.891$

$-73.776$

$-71.994$

70.023

$-64.318$

$-58.106$

$-56.757$

$-44.775$

$-40.894$

$-39.661$

38.059

$-28.581$

$-27.038$

$-26.075$

24.833

$-24.228$

$-24.044$

$-19.366$

$\leftarrow 18.442$

-10.779
-8.398 

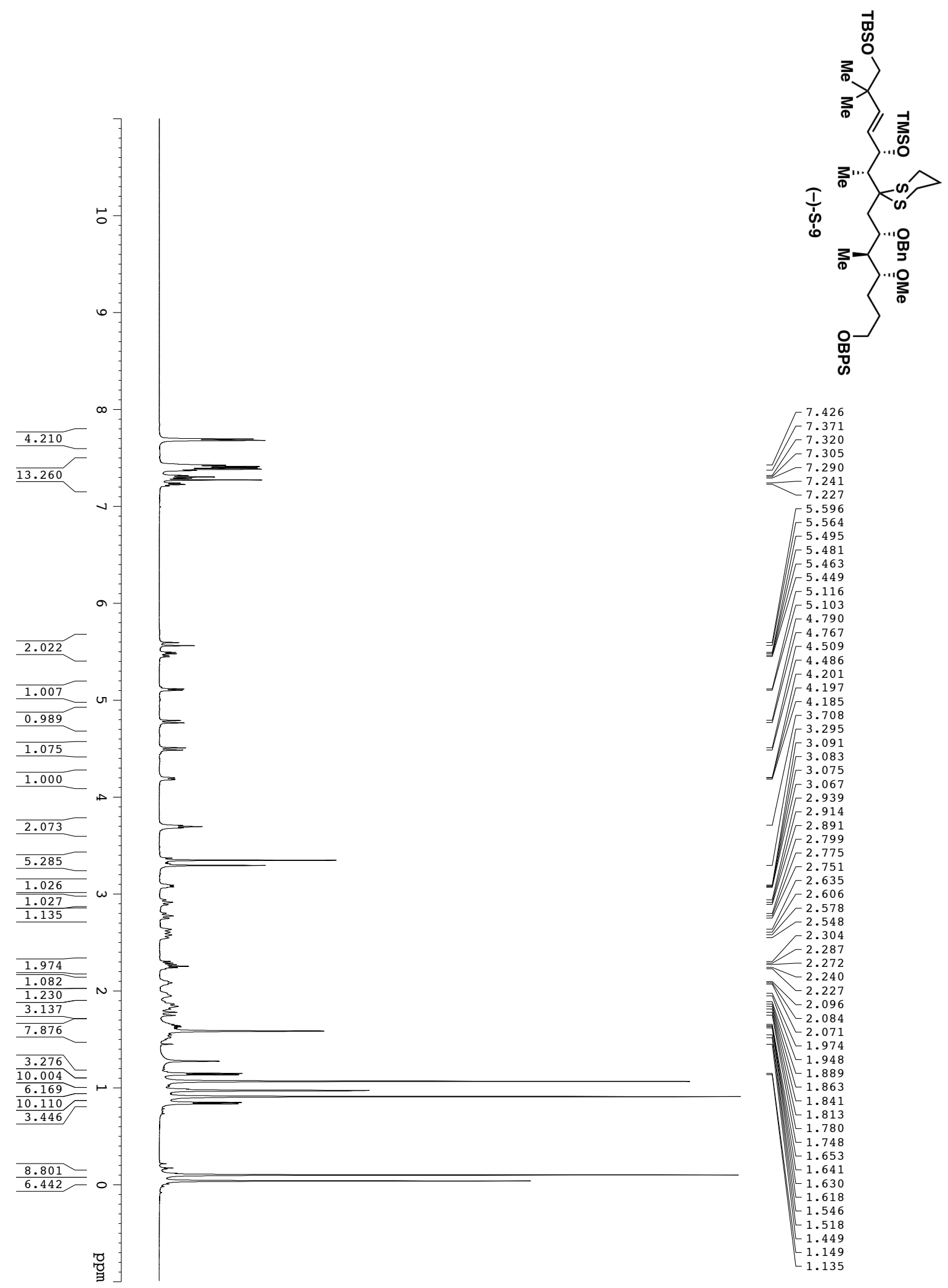

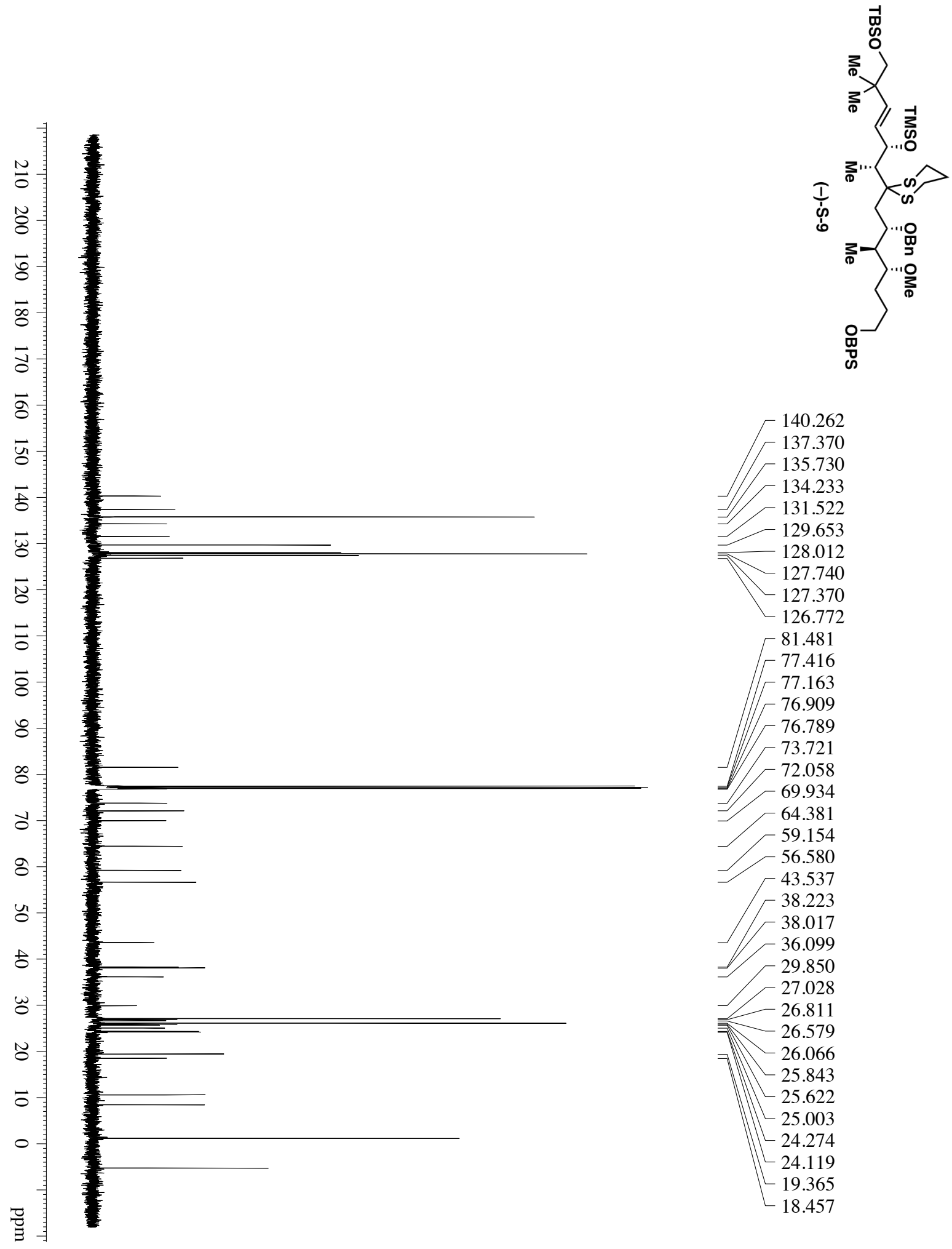

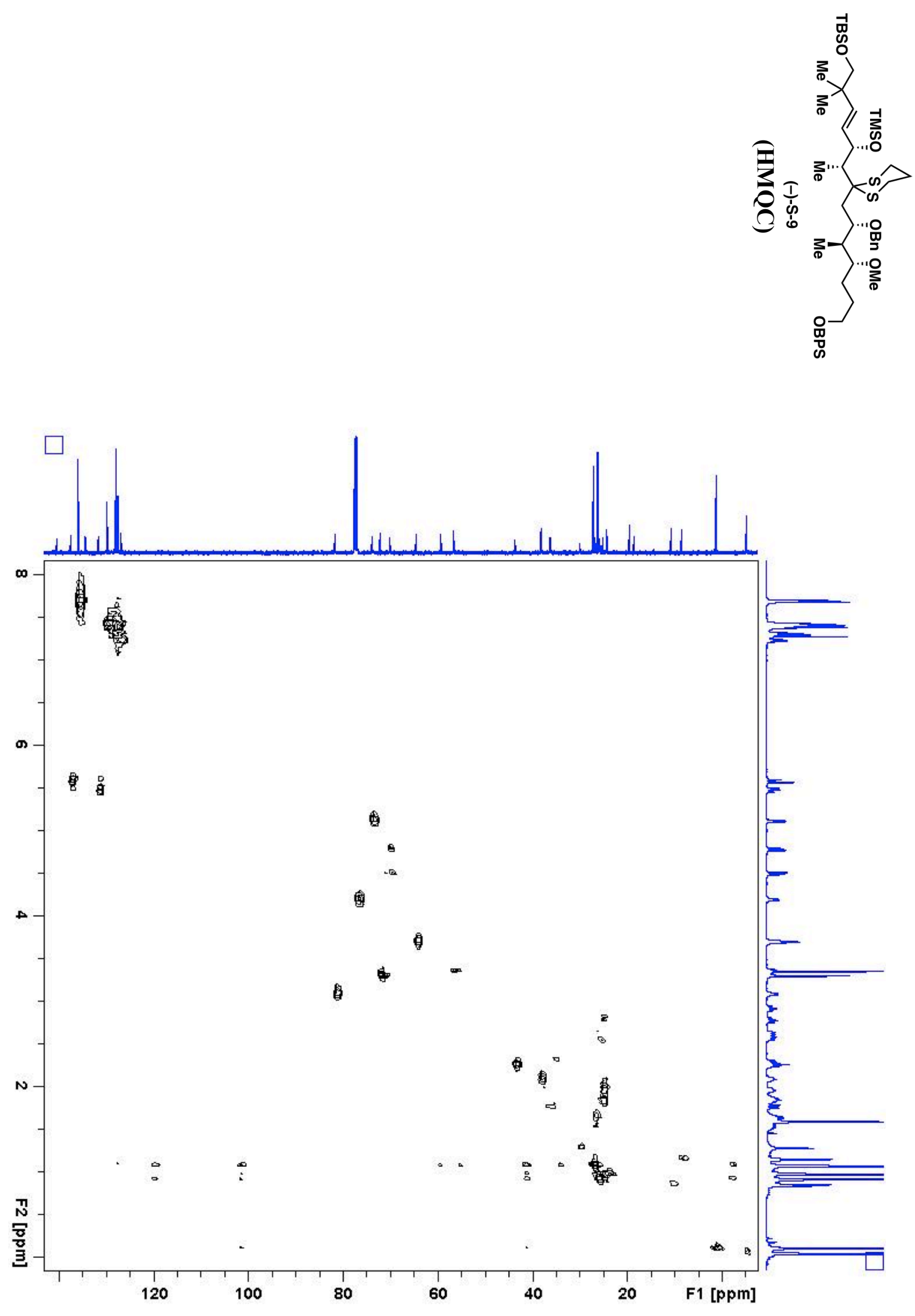

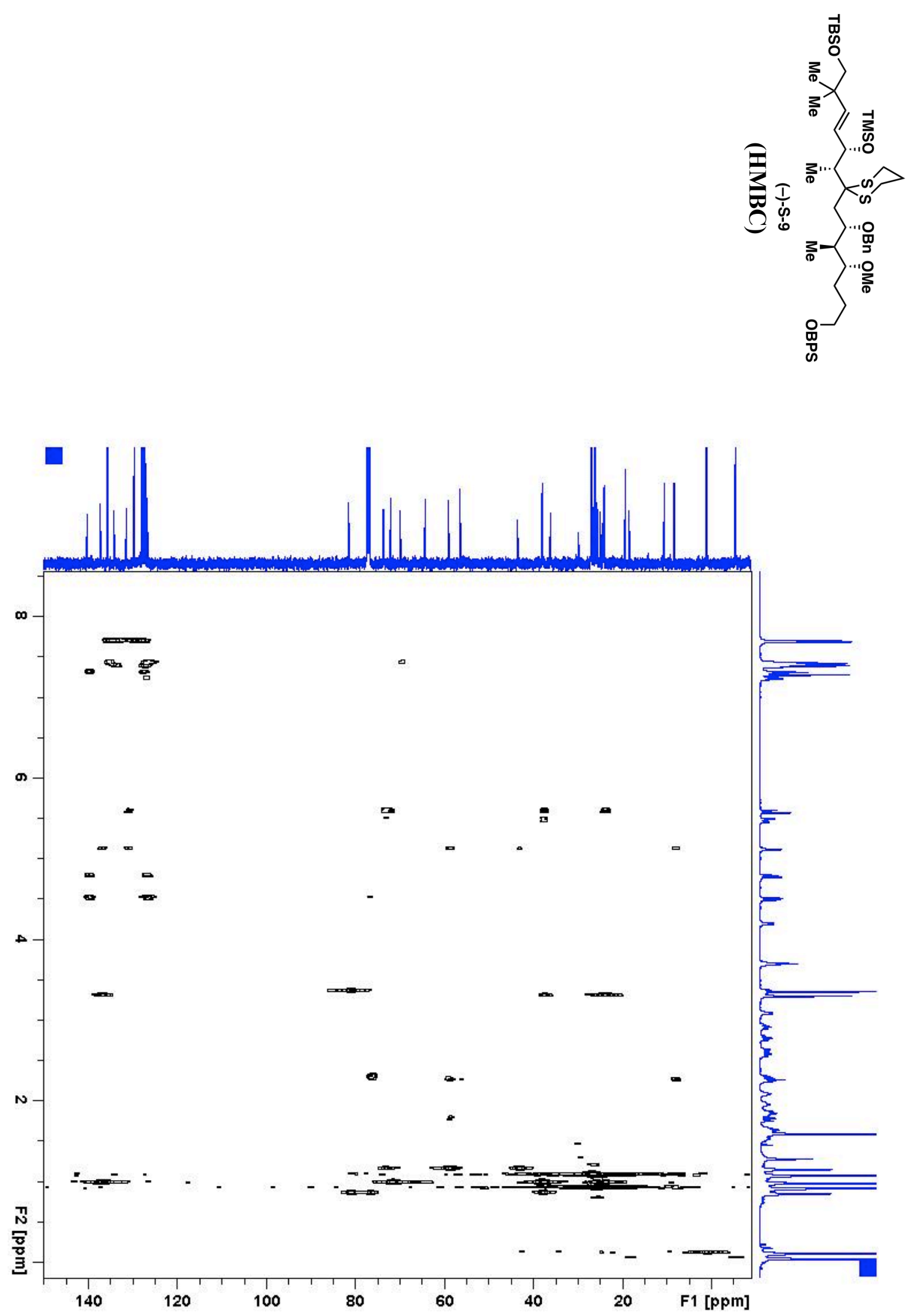


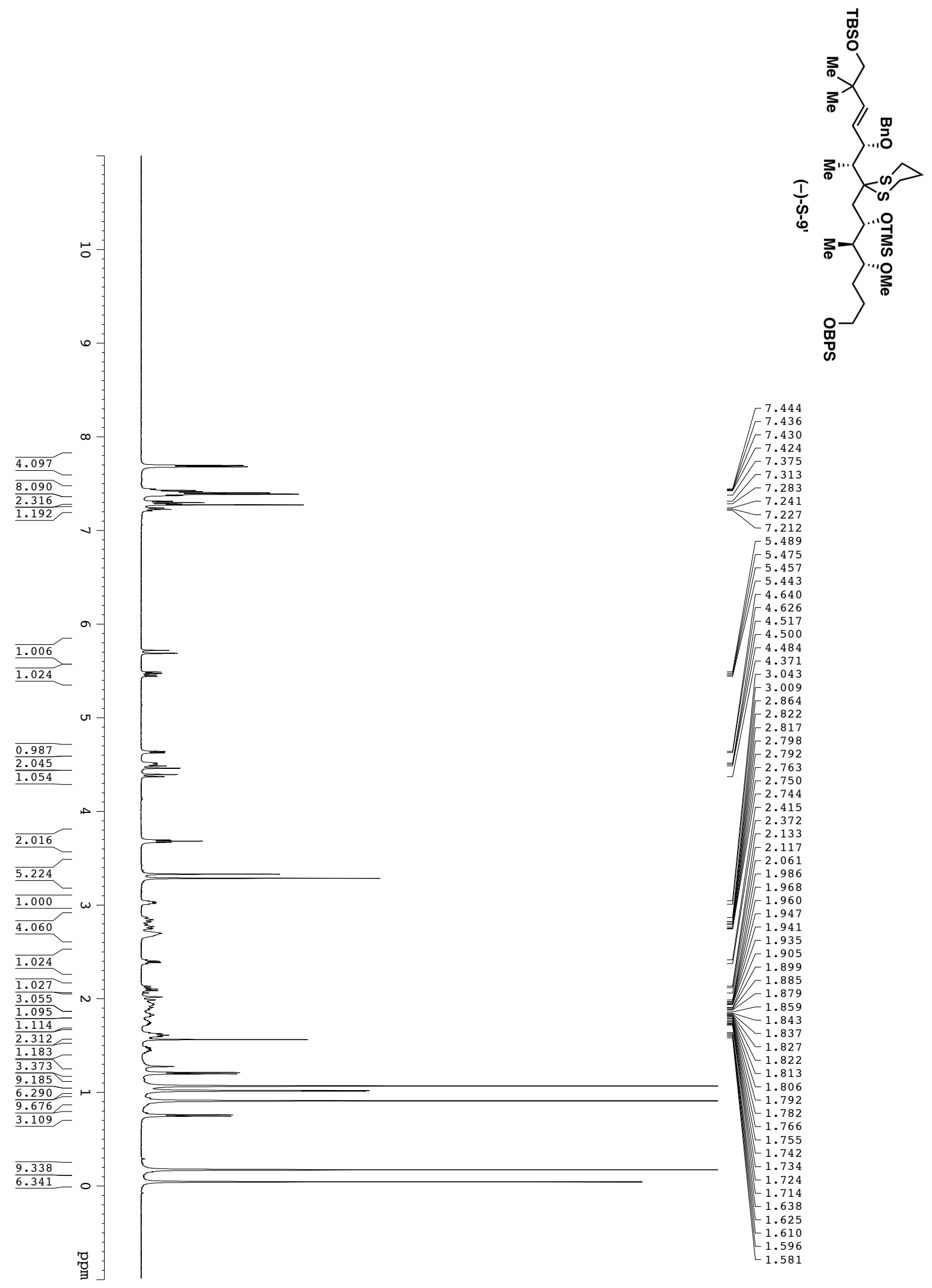



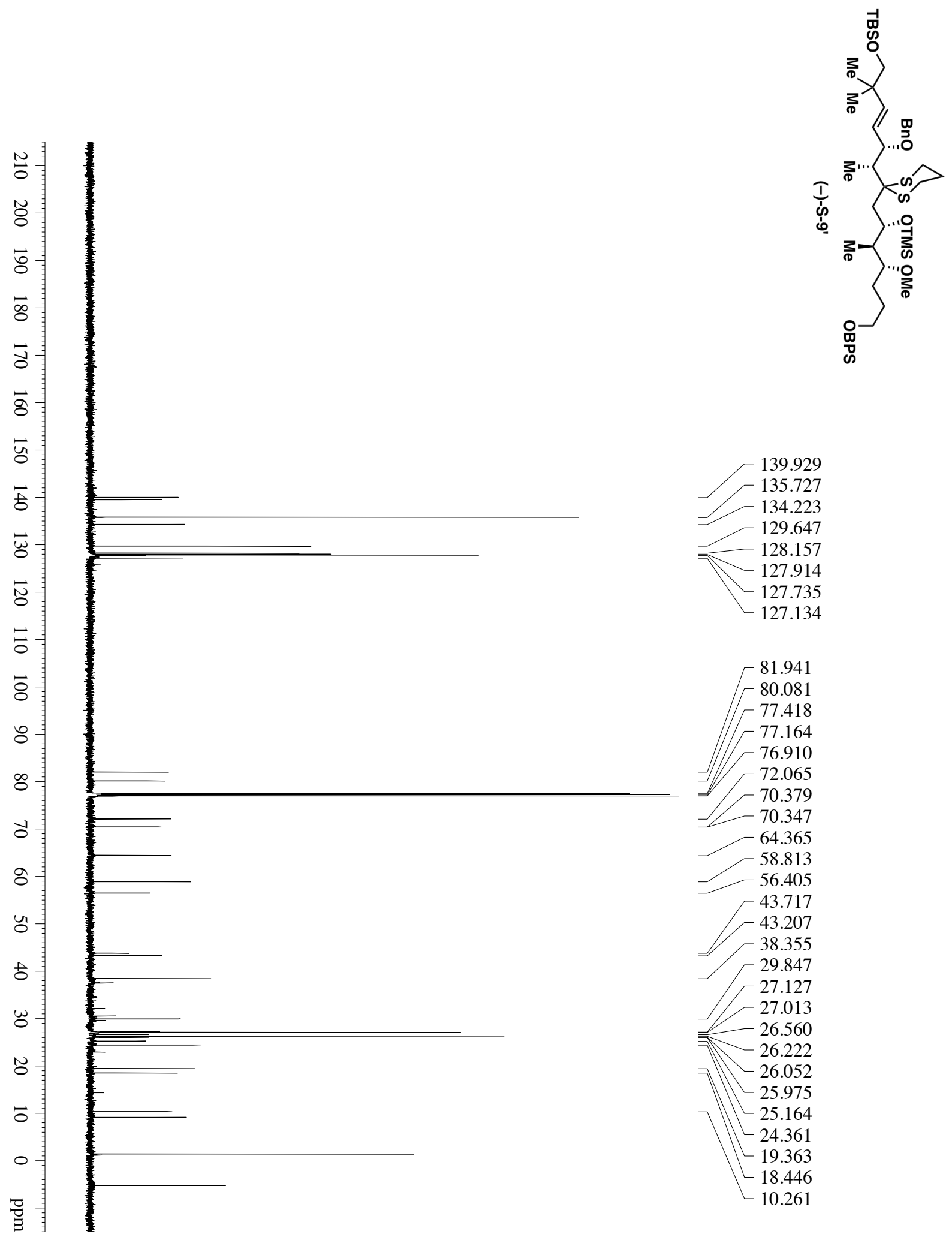


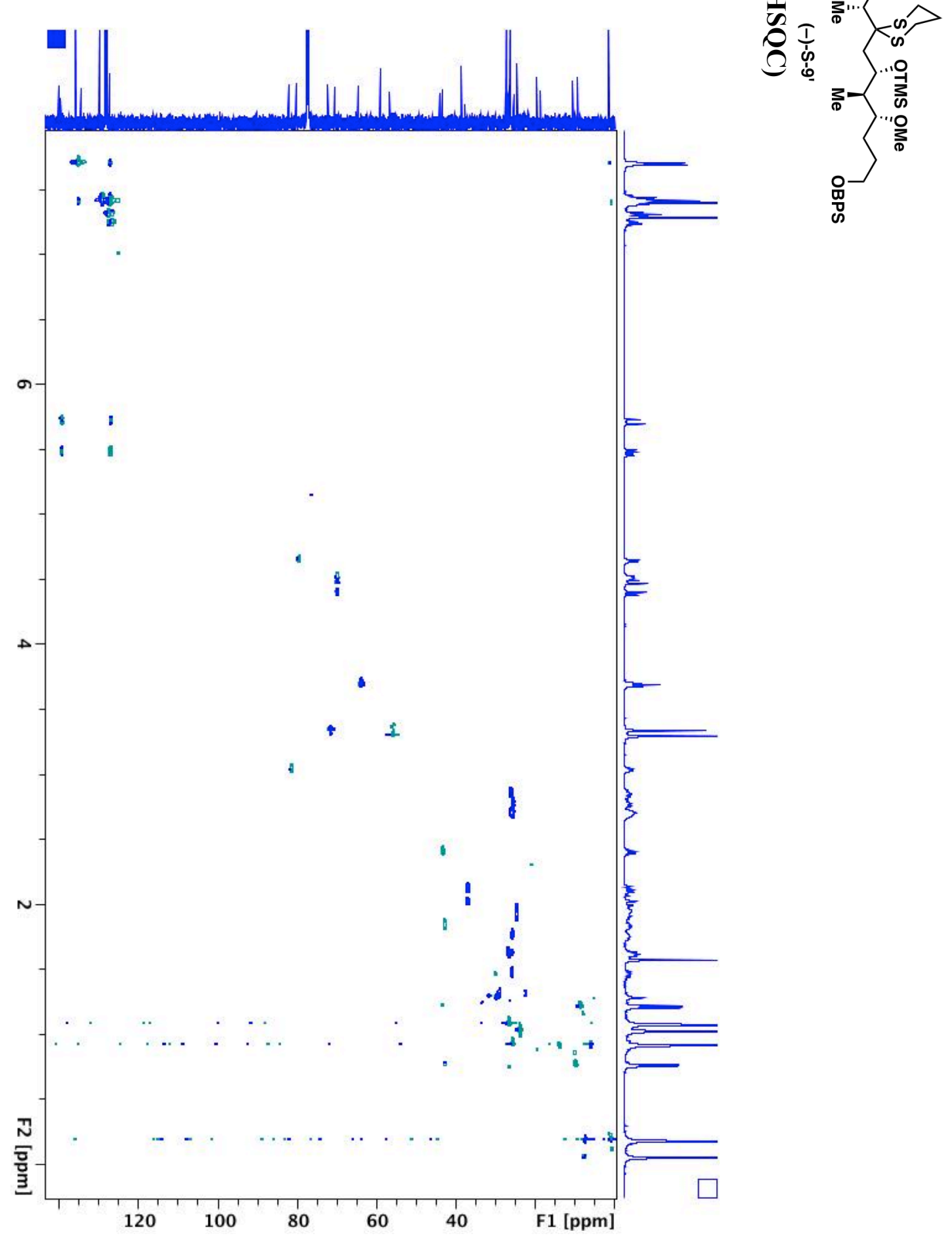



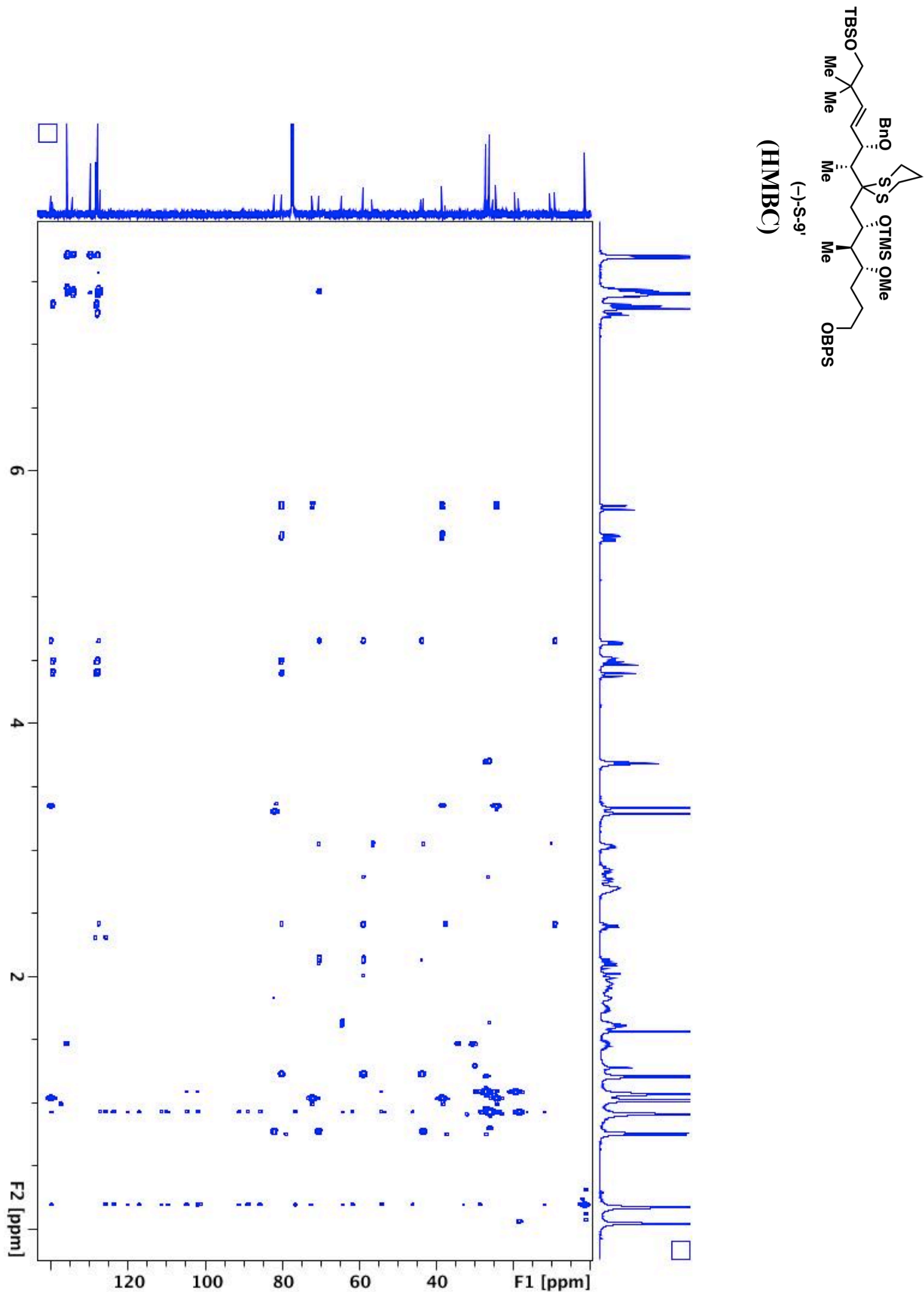


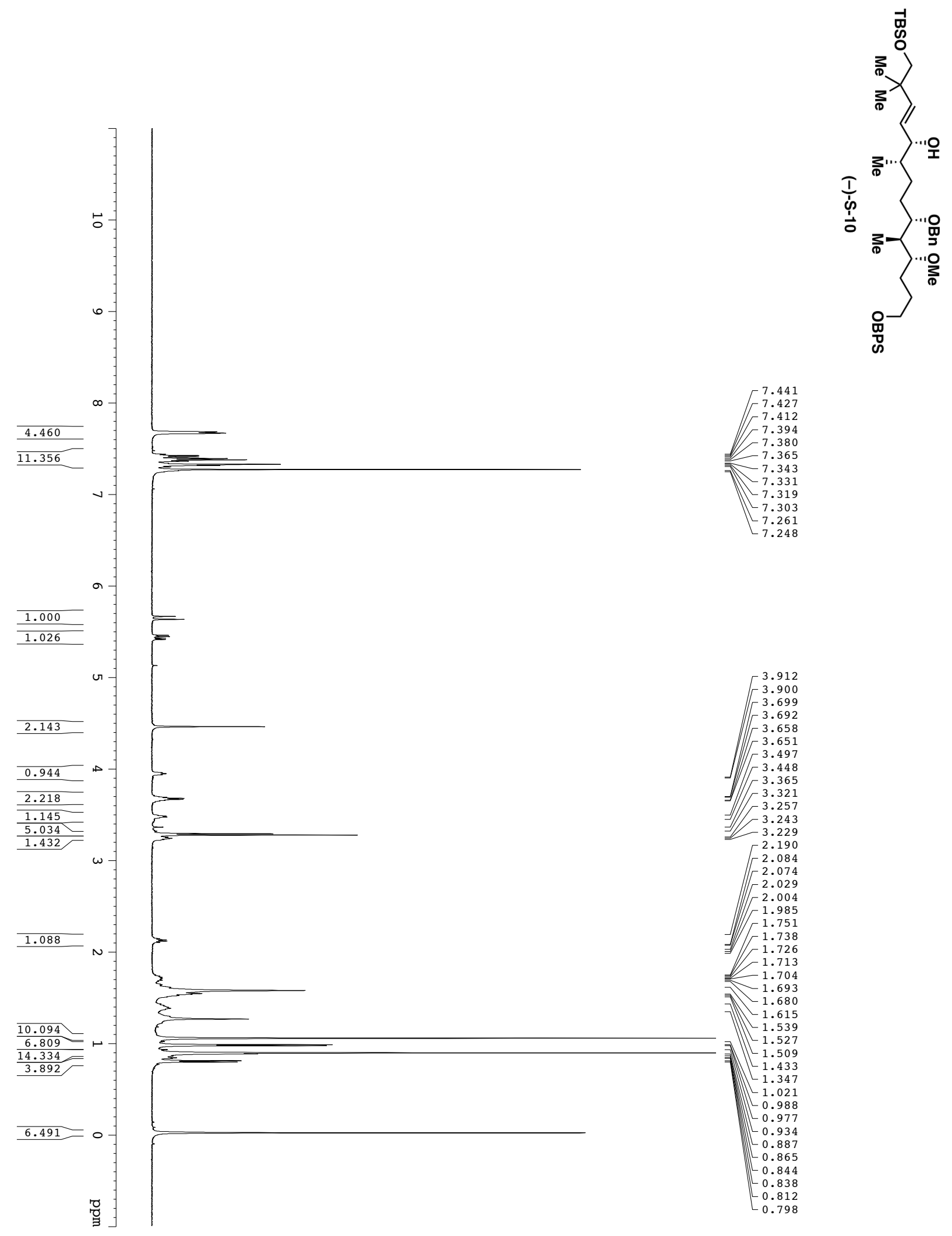



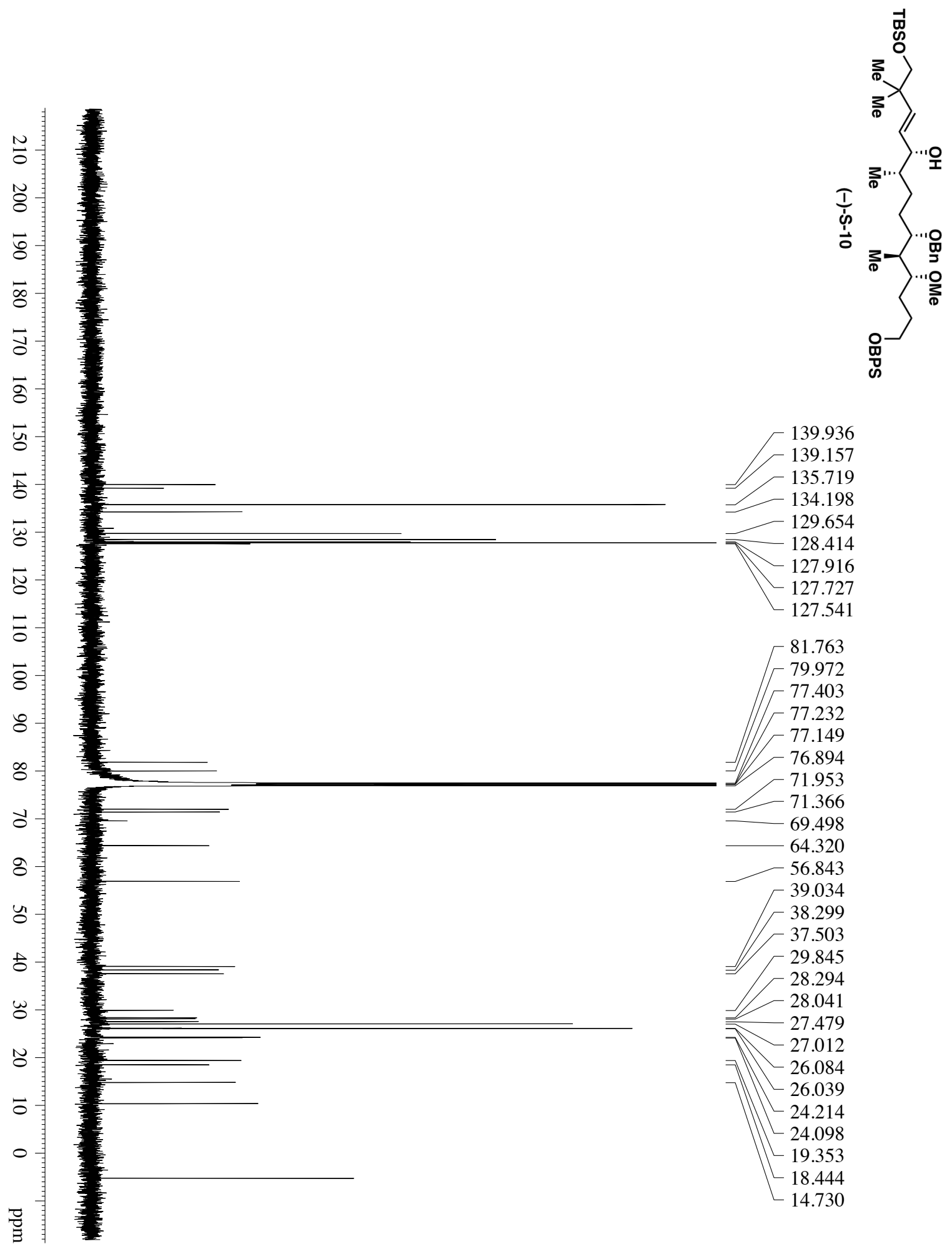
$-64.320$

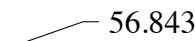

- 39.034

$-38.299$

$-37.503$

$-29.845$

$-28.294$

$-28.041$

27.479

$-26.084$

26.039

$-24.214$

$-24.098$

$-19.353$

$-18.444$

$-14.730$ 


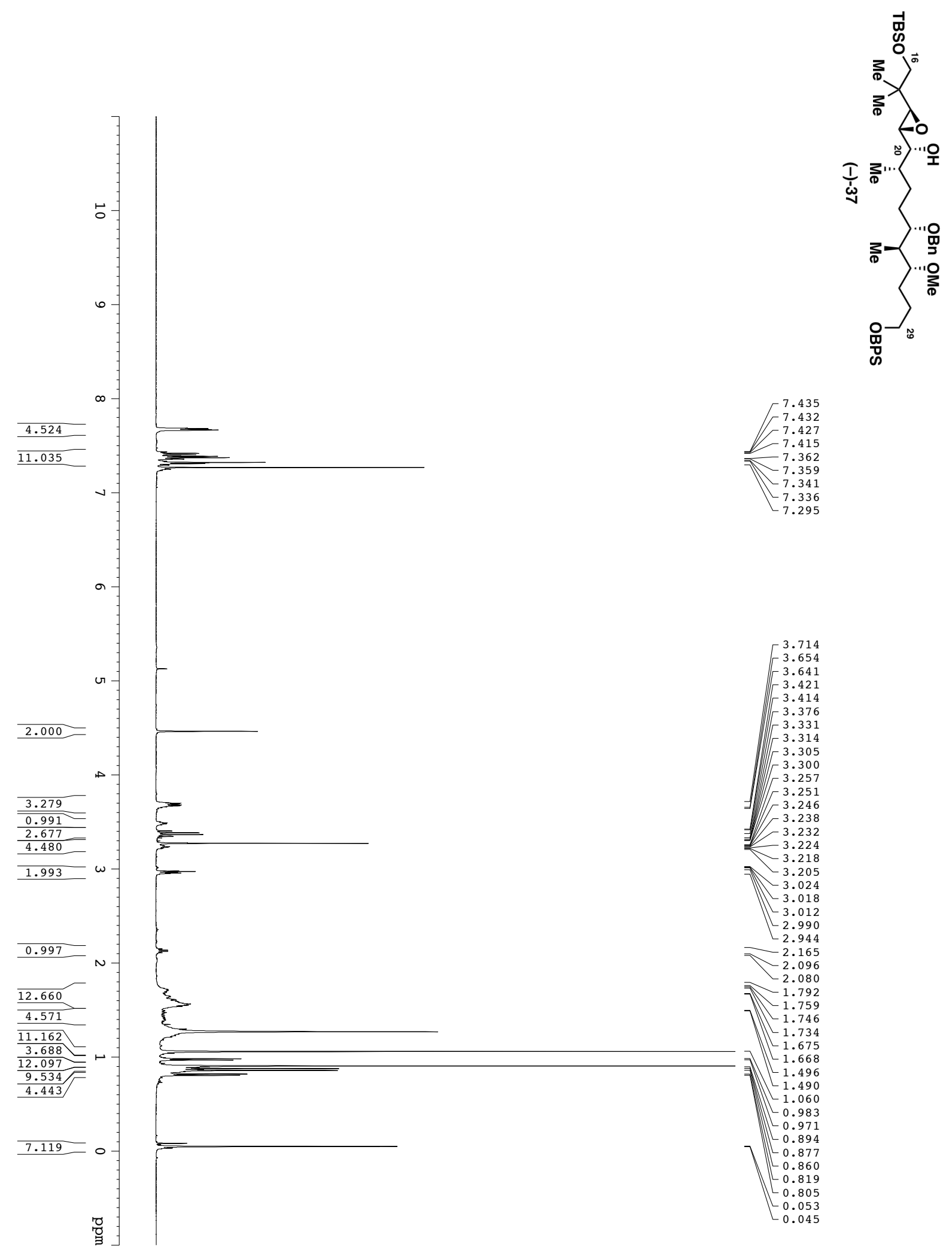




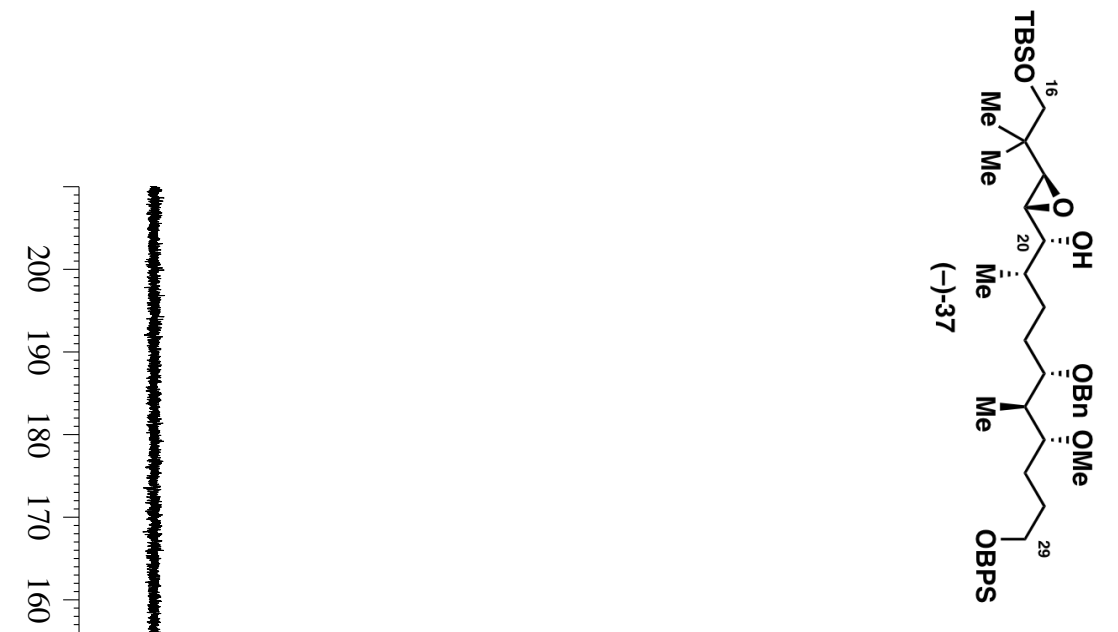

ğ

咠

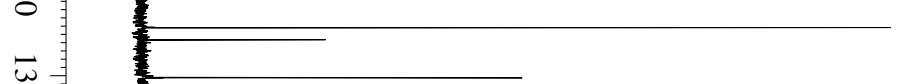

फ్

స్

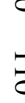

$\bar{\sigma}$

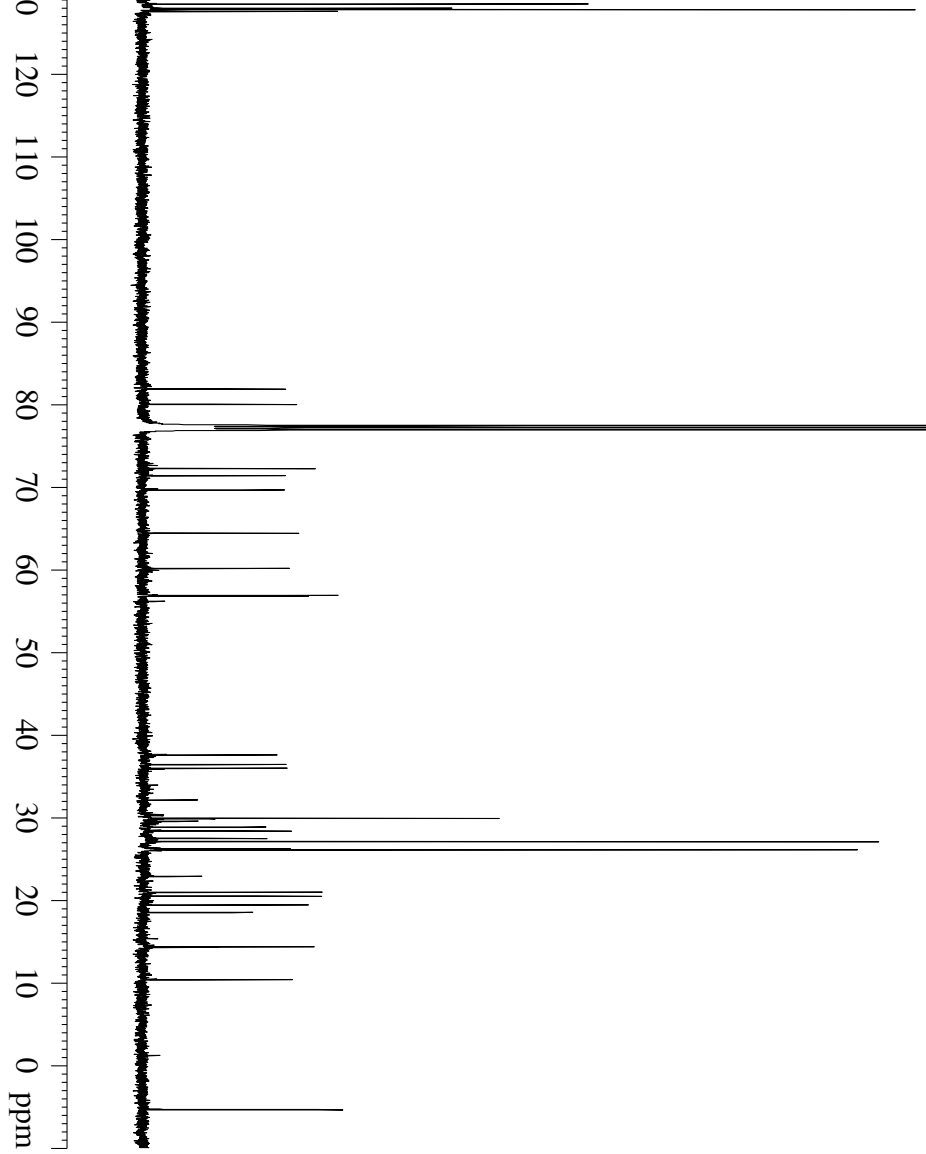

8

$\infty$

o

$a$

y

t

w

ธิ

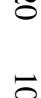

$\circ$

뭉

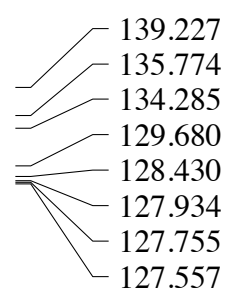

- 81.849

$-79.984$

$\mp 72.204$

工 71.350

- 69.603

64.384
-60.117

$-56.862$

$-56.763$

厂 37.570

36.400

$-32.093$

$-29.870$

$=28.838$

$-28.361$

$-27.448$

$-27.071$

刃) -26.198

$-26.079$

$-22.844$

$-20.959$

$-20.482$ 
${ }^{1}$ Love, B.; Jones, E. J. Org. Chem. 1999, 64, 3755.

${ }^{2}$ Ley, S. V.; Tackett, M. N.; Maddess, M. L.; Anderson, J. C.; Brennan, P. E.; Cappi, M. W.; Heer, J. P.; Helgen, C.; Kori, M.; Kouklovsky, C.; Marsden, S. P.; Norman, J.; Osborn, D. P.; Palomero, M. A.; Pavey, J. B.; J.; Pinel, C.; Robinson, L. A.; Schnaubelt, J.; Scott, J. S.; Spilling, C. D.; Watanabe, H.; Wesson, K. E.; Willis, M. C. Total synthesis of rapamycin. Chem. Eur. J. 2009, 15, 2874.

${ }^{3}$ Seyferth, D.; Weiner, M. A. J. Org. Chem. 1961, 26, 4797.

${ }^{4}$ Hoye, T. R.; Jeffrey, C. S.; Shao, F. Nat. Protoc. 2007, 2, 2451.

${ }^{5}$ Smith, A. B., III; Jurica, J. A.; Walsh, S. P. Org. Lett. 2008, 10, 5625.

${ }^{6}$ Takai, K.; Nitta, K.; Utimoto, K. J. Am. Chem. Soc. 1986, 108, 7408.

${ }^{7}$ Nicolaou, K. C.; Jiang, X.; Lindsay-Scott, P. J. Corbu, A.; Yamashiro, S.; Bacconi, A.; Fowler, V. M. Angew. Chem. Int. Ed. 2011, 50, 1139.

${ }^{8}$ Bartlett, P. A.; Meadows, J. D.; Brown, E. G.; Morimoto, A.; Jernstedt, K. K. J. Org. Chem. 1982, 47, 4013.

9 (a) Katsuki, T.; Sharpless, K. B. J. Am. Chem. Soc. 1980, 102, 5974; (b) Gollner, A.; Mulzer, J. Org. Lett. 2008, 10, 4701. 